Supporting Information

\title{
Design, Synthesis and Antifungal Evaluation of Neocryptolepine Derivatives against Phytopathogenic Fungi
}

Jia-Kai Zhu, Jian-Mei Gao, Cheng-Jie Yang, Xiao-Fei Shang, Zhong-Min Zhao, Raymond Kobla Lawoe, Rui Zhou, Yu Sun, Xiao-Dan Yin, Ying-Qian Liu*

School of Pharmacy, Lanzhou University, Lanzhou 730000, People's Republic of China

*Corresponding Author. (Phone: +86-898-66969260; fax: +86-898-66969211; e-mail: yqliu@1zu.edu.cn (Y. Q. Liu)) 


\section{Content}

1. In vitro antifungal activities of Neocryptolepine and its derivatives against six phytopathogenic fungi at $10 \mu \mathrm{g} / \mathrm{mL}$.

2. In vitro $\mathrm{EC}_{50}$ values of all synthetic compounds against six phytopathogenic fungi in detail.

3. Physical properties of the target compounds.

4. Spectrums of the representative compounds. 


\section{In vitro antifungal activities of Neocryptolepine and its derivatives against six}

phytopathogenic fungi at $10 \mu \mathrm{g} / \mathrm{mL}$.

\begin{tabular}{|c|c|c|c|c|c|c|}
\hline \multirow[b]{2}{*}{ Compound } & \multicolumn{6}{|c|}{ Inhibition rate $(\%)$} \\
\hline & S.s. & R.s. & B.c. & F.g. & M.o. & M.m. \\
\hline 1 & $71.68 \pm 1.64$ & $56.15 \pm 0.97$ & $45.14 \pm 0.26$ & $35.20 \pm 1.34$ & $38.58 \pm 0.85$ & $90.84 \pm 0.45$ \\
\hline 2 & $81.67 \pm 0.92$ & $45.50 \pm 0.41$ & $99.84 \pm 0.04$ & $91.47 \pm 0.49$ & $75.81 \pm 1.21$ & $99.71 \pm 0.16$ \\
\hline 3 & $80.95 \pm 1.01$ & $54.71 \pm 0.86$ & $100 \pm 0$ & $80.4 \pm 3.53$ & $92.69 \pm 0.71$ & $97.93 \pm 0.34$ \\
\hline 4 & $70.22 \pm 0.65$ & - & $87.2 \pm 0.40$ & $37.43 \pm 4.32$ & $30.43 \pm 1.75$ & $92.65 \pm 0.61$ \\
\hline 5 & $75.93 \pm 0.74$ & $39.69 \pm 0.77$ & $99.52 \pm 0.01$ & $86.00 \pm 0.66$ & $67.70 \pm 2.58$ & $99.58 \pm 0.29$ \\
\hline 6 & $84.79 \pm 0.59$ & - & $93.83 \pm 0.62$ & $67.10 \pm 1.43$ & $83.01 \pm 1.37$ & $98.42 \pm 0.01$ \\
\hline 7 & $60.59 \pm 0.61$ & $50.52 \pm 0.37$ & $96.52 \pm 0.97$ & $61.53 \pm 1.08$ & $53.02 \pm 1.67$ & $92.51 \pm 0.68$ \\
\hline 8 & $67.93 \pm 0.5$ & - & $93.74 \pm 0.33$ & $42.28 \pm 0.31$ & $46.09 \pm 1.15$ & $88.27 \pm 0.70$ \\
\hline 9 & - & $46.84 \pm 0.82$ & $83.84 \pm 0.99$ & $44.08 \pm 1.46$ & $31.35 \pm 0.67$ & $87.85 \pm 1.06$ \\
\hline 10 & $76.09 \pm 1.05$ & $54.93 \pm 0.74$ & $91.71 \pm 0.57$ & $52.96 \pm 0.57$ & $39.51 \pm 5.09$ & $95.01 \pm 0.46$ \\
\hline 11 & $63.67 \pm 0.81$ & - & $85.05 \pm 0.70$ & $33.62 \pm 1.05$ & $48.34 \pm 0.87$ & $94.85 \pm 0.42$ \\
\hline 12 & - & - & $70.13 \pm 0.85$ & $19.40 \pm 1.86$ & $13.71 \pm 0.62$ & $54.43 \pm 0.99$ \\
\hline 13 & $42.20 \pm 0.59$ & $29.80 \pm 0.97$ & - & $21.23 \pm 1.38$ & $27.22 \pm 3.66$ & $55.64 \pm 0.36$ \\
\hline 14 & $42.45 \pm 0.93$ & $26.69 \pm 1.23$ & $94.33 \pm 0.74$ & $59.50 \pm 2.59$ & $57.70 \pm 1.10$ & $83.36 \pm 0.36$ \\
\hline 15 & - & - & $66.63 \pm 1.54$ & - & $38.83 \pm 0.97$ & $65.92 \pm 0.91$ \\
\hline 16 & $74.80 \pm 0.71$ & $39.97 \pm 0.69$ & $97.27 \pm 0.23$ & $75.50 \pm 0.76$ & $72.62 \pm 1.06$ & $93.33 \pm 0.62$ \\
\hline 17 & $55.19 \pm 0.42$ & $35.52 \pm 1.04$ & $87.91 \pm 0.12$ & $64.38 \pm 0.94$ & $53.37 \pm 2.29$ & $74.30 \pm 0.79$ \\
\hline 18 & $48.20 \pm 0.93$ & $41.01 \pm 0.29$ & $84.65 \pm 1.11$ & $55.53 \pm 0.77$ & $18.04 \pm 0.46$ & $83.95 \pm 0.80$ \\
\hline 19 & $70.40 \pm 1.36$ & $55.55 \pm 1.31$ & $92.89 \pm 0.53$ & $65.39 \pm 0.61$ & $66.32 \pm 0.30$ & $94.10 \pm 0.32$ \\
\hline 20 & $80.18 \pm 0.47$ & - & $86.03 \pm 0.93$ & $65.70 \pm 0.31$ & $84.38 \pm 0.71$ & $95.49 \pm 0.03$ \\
\hline 21 & $77.05 \pm 0.89$ & $38.63 \pm 0.60$ & $96.44 \pm 0.54$ & $83.22 \pm 0.74$ & $75.48 \pm 2.30$ & $100 \pm 0$ \\
\hline 22 & $61.18 \pm 0.51$ & - & $96.70 \pm 0.46$ & $55.68 \pm 1.26$ & $53.73 \pm 2.13$ & $93.85 \pm 0.18$ \\
\hline 23 & $76.62 \pm 1.05$ & $70.68 \pm 0.60$ & $96.62 \pm 0.38$ & $55.74 \pm 1.26$ & $40.29 \pm 0.31$ & $94.81 \pm 0.50$ \\
\hline 24 & $88.89 \pm 0.55$ & $65.29 \pm 0.81$ & $97.15 \pm 0.08$ & $63.08 \pm 0.34$ & $70.70 \pm 2.01$ & $100 \pm 0$ \\
\hline 25 & $99.75 \pm 0.12$ & $76.57 \pm 1.37$ & $95.75 \pm 0.37$ & $58.01 \pm 0.77$ & $63.50 \pm 1.55$ & $100 \pm 0$ \\
\hline 26 & $55.04 \pm 1.20$ & - & $83.48 \pm 0.62$ & $50.50 \pm 0.97$ & $41.68 \pm 0.42$ & $70.43 \pm 1.99$ \\
\hline 27 & - & - & $70.52 \pm 1.33$ & $36.51 \pm 0.53$ & - & $57.90 \pm 1.23$ \\
\hline 28 & $70.18 \pm 1.99$ & - & $71.26 \pm 1.26$ & - & - & $80.78 \pm 0.45$ \\
\hline 29 & $46.37 \pm 0.67$ & - & $46.57 \pm 1.85$ & - & $48.24 \pm 0.35$ & $77.01 \pm 0.40$ \\
\hline 30 & $43.30 \pm 0.31$ & - & $66.36 \pm 1.29$ & - & - & $74.33 \pm 0.77$ \\
\hline 31 & - & - & $66.63 \pm 1.54$ & $21.23 \pm 1.02$ & - & $41.44 \pm 2.21$ \\
\hline 32 & - & - & - & - & - & - \\
\hline 33 & - & - & - & - & - & - \\
\hline 34 & $14.95 \pm 1.15$ & $22.21 \pm 1.01$ & $91.71 \pm 3.98$ & $38.65 \pm 2.05$ & $57.98 \pm 2.85$ & $84.16 \pm 0.36$ \\
\hline 35 & $79.18 \pm 0.61$ & $68.53 \pm 0.67$ & $95.83 \pm 1.23$ & $83.74 \pm 0.81$ & $100 \pm 0$ & $100 \pm 0$ \\
\hline 36 & $70.52 \pm 1.04$ & $38.88 \pm 1.67$ & $96.08 \pm 0.76$ & $81.72 \pm 1.01$ & $95.19 \pm 0.60$ & $92.75 \pm 0.35$ \\
\hline 37 & $73.07 \pm 1.96$ & $33.50 \pm 1.23$ & $95.17 \pm 0.66$ & $78.13 \pm 0.94$ & $83.74 \pm 0.91$ & $91.65 \pm 0.31$ \\
\hline 38 & $73.48 \pm 1.83$ & $46.03 \pm 1.15$ & $95.20 \pm 0.09$ & $78.34 \pm 1.06$ & $95.49 \pm 0.65$ & $90.98 \pm 0.12$ \\
\hline
\end{tabular}




\begin{tabular}{ccccccc}
39 & $69.37 \pm 0.73$ & $31.05 \pm 1.06$ & $95.38 \pm 0.60$ & $66.90 \pm 0.67$ & $81.68 \pm 0.83$ & $87.52 \pm 0.78$ \\
40 & $77.14 \pm 0.38$ & $68.95 \pm 0.89$ & $97.92 \pm 0.23$ & $75.59 \pm 2.66$ & $91.83 \pm 0.54$ & $93.2 \pm 0.39$ \\
41 & $74.73 \pm 0.41$ & $40.60 \pm 0.73$ & $95.19 \pm 0.28$ & $86.12 \pm 0.42$ & $88.45 \pm 1.73$ & $92.9 \pm 0.06$ \\
42 & $76.15 \pm 1.00$ & $36.70 \pm 0.84$ & $95.46 \pm 0.23$ & $76.59 \pm 1.48$ & $90.72 \pm 0.23$ & $92.35 \pm 0.36$ \\
43 & $71.45 \pm 0.75$ & $35.50 \pm 2.14$ & $96.38 \pm 0.19$ & $76.65 \pm 1.64$ & $99.53 \pm 0.40$ & $93.14 \pm 0.20$ \\
44 & $73.64 \pm 0.84$ & $24.09 \pm 0.93$ & $96.34 \pm 0.07$ & $75.50 \pm 1.92$ & $89.04 \pm 1.34$ & $91.61 \pm 0.56$ \\
45 & $75.27 \pm 1.42$ & $68.81 \pm 1.56$ & $95.93 \pm 0.35$ & $56.50 \pm 2.35$ & $65.30 \pm 1.45$ & $91.73 \pm 0.83$ \\
46 & $57.04 \pm 1.10$ & $23.20 \pm 1.42$ & $92.52 \pm 0.64$ & $49.66 \pm 0.79$ & $52.50 \pm 0.79$ & $87.82 \pm 0.67$ \\
47 & $50.34 \pm 1.16$ & $23.58 \pm 1.94$ & $90.34 \pm 0.36$ & $42.04 \pm 0.59$ & $47.83 \pm 0.14$ & $78.81 \pm 0.12$ \\
48 & $73.48 \pm 0.70$ & $36.78 \pm 0.23$ & $96.90 \pm 0.58$ & $83.80 \pm 0.88$ & $94.66 \pm 0.60$ & $92.68 \pm 0.24$ \\
49 & $56.90 \pm 0.82$ & $30.60 \pm 1.21$ & $96.44 \pm 0.06$ & $59.11 \pm 0.95$ & $73.15 \pm 1.44$ & $89.95 \pm 0.69$ \\
ASB & $14.31 \pm 1.66$ & $34.91 \pm 0.27$ & $18.28 \pm 0.46$ & $44.68 \pm 0.76$ & $31.28 \pm 0.34$ & $45.03 \pm 0.93$ \\
\hline
\end{tabular}

S.s., Sclerotinia sclerotiorum ; R.s., Rhizoctonia solani; B.c., Botrytis cinerea; F.g., Fusarium graminearum; M.o.,

Magnaporthe oryzae; M.m., Mycosphaerlla melonis. ASB, azoxystrobin. “-” means not test.

\section{In vitro $\mathrm{EC}_{50}$ values of all synthetic compounds against six phytopathogenic}

\section{fungi in detail.}

\begin{tabular}{|c|c|c|c|c|c|}
\hline \multirow[b]{2}{*}{ compound } & \multicolumn{5}{|c|}{ Sclerotinia sclerotiorum } \\
\hline & $\begin{array}{c}\mathrm{EC}_{50} \\
(\mu \mathrm{g} / \mathrm{mL})\end{array}$ & $95 \% \mathrm{CI}$ & Regression equation & $\mathrm{R}^{2}$ & $\begin{array}{c}\text { Chi-square } \\
\text { value }\end{array}$ \\
\hline 1 & 17.65 & $14.198-22.703$ & $\mathrm{Y}=-1.58+1.27 \mathrm{X}$ & 0.989 & 1.110 \\
\hline 2 & 4.01 & $3.371-4.643$ & $Y=-1.39+2.3 X$ & 0.991 & 1.397 \\
\hline 3 & 3.72 & $0.384-6.911$ & $Y=-1.1+1.93 X$ & 0.949 & 5.819 \\
\hline 4 & 6.21 & $0.119-34.010$ & $Y=-1.91+2.34 \mathrm{X}$ & 0.926 & 11.327 \\
\hline 5 & 5.03 & $4.412-5.698$ & $Y=-2.08+3 X$ & 0.982 & 2.17 \\
\hline 6 & 2.96 & $1.927-3.922$ & $\mathrm{Y}=-0.58+1.25 \mathrm{X}$ & 0.939 & 2.655 \\
\hline 7 & 6.59 & $1.047-11.491$ & $Y=-2.4+2.96 X$ & 0.969 & 6.422 \\
\hline 8 & 6.01 & $0.103-12.034$ & $Y=-1.15+1.5 X$ & 0.925 & 4.761 \\
\hline 9 & $>30$ & - & - & - & - \\
\hline 10 & 3.16 & $1.651-4.556$ & $Y=-0.44+0.89 X$ & 0.912 & 2.443 \\
\hline 11 & 3.97 & $1.523-6.358$ & $Y=-0.54+0.9 X$ & 0.966 & 0.850 \\
\hline 12 & $>30$ & - & - & - & - \\
\hline 13 & 15.59 & $12.054-20.137$ & $\mathrm{Y}=-1.43+1.21 \mathrm{X}$ & 0.961 & 2.036 \\
\hline 14 & 12.98 & $1.943-42.417$ & $Y=-2.32+2.12 \mathrm{X}$ & 0.932 & 8.431 \\
\hline 15 & $>30$ & - & - & - & - \\
\hline 16 & 3.70 & $2.741-4.638$ & $Y=-0.83+1.46 X$ & 0.971 & 1.964 \\
\hline 17 & 6.10 & $3.643-8.420$ & $\mathrm{Y}=-0.85+1.08 \mathrm{X}$ & 0.953 & 1.828 \\
\hline
\end{tabular}




\begin{tabular}{|c|c|c|c|c|c|}
\hline 18 & 8.98 & $6.951-11.031$ & $Y=-1.45+1.53 X$ & 0.960 & 2.866 \\
\hline 19 & 4.26 & $2.751-5.750$ & $Y=-0.63+X$ & 0.908 & 3.237 \\
\hline 20 & 5.04 & $0.015-13.697$ & $Y=-1.02+1.44 X$ & 0.917 & 5.793 \\
\hline 21 & 1.07 & $0.413-1.696$ & $\mathrm{Y}=-0.02+0.8 \mathrm{X}$ & 0.989 & 0.205 \\
\hline 22 & 5.77 & $0.002-2.722$ & $\mathrm{Y}=0.05+0.54 \mathrm{X}$ & 0.942 & 0.574 \\
\hline 23 & 4.03 & $2.732-5.295$ & $Y=-0.68+1.13 X$ & 0.939 & 2.696 \\
\hline 24 & 2.80 & $0.018-16.437$ & $Y=-1+2.27 X$ & 0.925 & 12.261 \\
\hline 25 & 2.90 & $2.639-3.190$ & $Y=-2.01+4.61 X$ & 0.988 & 1.258 \\
\hline 26 & 10.67 & $6.793-14.826$ & $Y=-0.91+0.88 X$ & 0.928 & 2.182 \\
\hline 27 & $>30$ & - & - & - & - \\
\hline 28 & 5.41 & $2.812-22.997$ & $Y=-1.55+2.09 X$ & 0.946 & 6.334 \\
\hline 29 & 12.49 & 9.879-15.404 & $Y=-1.56+1.42 X$ & 0.991 & 0.633 \\
\hline 30 & 14.42 & $8.791-22.509$ & $\mathrm{Y}=-0.81+0.7 \mathrm{X}$ & 0.980 & 0.358 \\
\hline 31 & $>30$ & - & - & - & - \\
\hline 32 & $>30$ & - & - & - & - \\
\hline 33 & $>30$ & - & - & - & - \\
\hline 34 & $>30$ & - & - & - & - \\
\hline 35 & 1.93 & $1.286-2.571$ & $Y=-0.31+1.07 X$ & 0.980 & 0.752 \\
\hline 36 & 2.97 & $2.312-3.742$ & $Y=-0.62+1.29 X$ & 0.940 & 3.434 \\
\hline 37 & 4.21 & $2.015-11.642$ & $\mathrm{Y}=-1.27+2.02 \mathrm{X}$ & 0.958 & 5.807 \\
\hline 38 & 4.74 & $2.450-14.962$ & $Y=-1.31+1.94 X$ & 0.949 & 5.463 \\
\hline 39 & 5.87 & $4.893-7.358$ & $Y=-1.32+1.71 X$ & 0.963 & 3.033 \\
\hline 40 & 2.68 & $0.679-5.816$ & $\mathrm{Y}=-0.7+1.61 \mathrm{X}$ & 0.943 & 4.742 \\
\hline 41 & 3.62 & $3.034-4.343$ & $Y=-0.98+1.74 X$ & 0.983 & 1.610 \\
\hline 42 & 4.08 & $1.447-28.196$ & $Y=-0.94+1.54 X$ & 0.928 & 5.529 \\
\hline 43 & 3.83 & $3.199-4.641$ & $Y=-0.99+1.68 X$ & 0.960 & 3.769 \\
\hline 44 & 4.87 & $1.916-39.351$ & $Y=-1.57+2.26 X$ & 0.938 & 9.141 \\
\hline 45 & 2.77 & $2.192-3.418$ & $Y=-0.63+1.41 X$ & 0.972 & 1.772 \\
\hline 46 & 5.16 & $3.849-7.734$ & $Y=-0.72+X$ & 0.932 & 2.532 \\
\hline 47 & 8.44 & $6.671-13.171$ & $\mathrm{Y}=-1.28+1.36 \mathrm{X}$ & 0.971 & 1.655 \\
\hline 48 & 3.52 & $2.923-4.247$ & $Y=-0.91+1.65 X$ & 0.959 & 3.674 \\
\hline 49 & 5.98 & $4.693-8.317$ & $Y=-1+1.29 X$ & 0.942 & 3.528 \\
\hline
\end{tabular}

\begin{tabular}{cccccc}
\hline & \multicolumn{5}{c}{ Rhizoctonia solani } \\
\cline { 2 - 6 } compound & $\begin{array}{c}\mathrm{EC}_{50} \\
(\mu \mathrm{g} / \mathrm{mL})\end{array}$ & $95 \% \mathrm{CI}$ & Regression equation & $\mathrm{R}^{2}$ & $\begin{array}{c}\text { Chi-square } \\
\text { value }\end{array}$ \\
\hline 1 & 9.00 & $7.540-10.691$ & $\mathrm{Y}=-1.59+1.67 \mathrm{X}$ & 0.985 & 2.557 \\
2 & 8.92 & $0.388-19.227$ & $\mathrm{Y}=-1.3+1.37 \mathrm{X}$ & 0.931 & 4.508 \\
3 & 7.11 & $0.422-13.918$ & $\mathrm{Y}=-2.96+3.42 \mathrm{X}$ & 0.927 & 7.797 \\
& & & & &
\end{tabular}




\begin{tabular}{|c|c|c|c|c|c|}
\hline 4 & $>30$ & - & - & - & _ \\
\hline 5 & 11.79 & $8.960-14.933$ & $\mathrm{Y}=-1.34+1.25 \mathrm{X}$ & 0.941 & 3.383 \\
\hline 6 & $>30$ & - & - & - & - \\
\hline 7 & 8.76 & $5.936-11.586$ & $Y=-1.03+1.09 X$ & 0.966 & 1.349 \\
\hline 8 & $>30$ & - & - & - & - \\
\hline 9 & 12.93 & $9.941-16.382$ & $\mathrm{Y}=-1.4+1.26 \mathrm{X}$ & 0.985 & 0.867 \\
\hline 10 & 6.68 & $3.731-9.466$ & $Y=-0.78+0.94 X$ & 0.981 & 0.557 \\
\hline 11 & $>30$ & - & - & - & - \\
\hline 12 & $>30$ & - & - & - & - \\
\hline 13 & 19.29 & $15.847-23.896$ & $Y=-1.96+1.52 X$ & 0.974 & 2.088 \\
\hline 14 & 13.48 & $11.030-16.289$ & $\mathrm{Y}=-1.8+1.59 \mathrm{X}$ & 0.962 & 3.266 \\
\hline 15 & $>30$ & - & - & - & - \\
\hline 16 & 12.87 & $9.109-17.328$ & $Y=-1.09+0.98 X$ & 0.917 & 3.040 \\
\hline 17 & 13.79 & $11.391-16.551$ & $\mathrm{Y}=-1.9+1.66 \mathrm{X}$ & 0.973 & 2.511 \\
\hline 18 & 14.04 & $10.737-18.050$ & $\mathrm{Y}=-1.36+1.19 \mathrm{X}$ & 0.993 & 0.369 \\
\hline 19 & 13.48 & $3.139-33.896$ & $Y=-0.46+0.41 X$ & 0.935 & 0.426 \\
\hline 20 & $>30$ & - & - & - & - \\
\hline 21 & 14.04 & $11.588-16.890$ & $Y=-1.9+1.66 \mathrm{X}$ & 0.997 & 0.245 \\
\hline 22 & $>25$ & & $\mathrm{Y}=+\mathrm{X}$ & & \\
\hline 23 & 3.52 & $2.517-4.521$ & $\mathrm{Y}=-0.7+1.29 \mathrm{X}$ & 0.979 & 1.350 \\
\hline 24 & 4.93 & $3.303-6.635$ & $Y=-0.68+0.98 X$ & 0.920 & 2.755 \\
\hline 25 & 5.45 & $4.095-7.004$ & $Y=-0.86+1.17 X$ & 0.970 & 1.662 \\
\hline 26 & $>30$ & - & - & - & - \\
\hline 27 & $>30$ & - & - & - & - \\
\hline 28 & $>30$ & - & - & - & - \\
\hline 29 & $>30$ & - & - & - & - \\
\hline 30 & $>30$ & - & - & - & - \\
\hline 31 & $>30$ & - & - & - & - \\
\hline 32 & $>30$ & - & - & - & - \\
\hline 33 & $>30$ & - & - & - & - \\
\hline 34 & $>30$ & - & - & - & - \\
\hline 35 & 5.29 & $3.962-6.788$ & $\mathrm{Y}=-0.85+1.18 \mathrm{X}$ & 0.961 & 2.110 \\
\hline 36 & $>30$ & - & - & - & - \\
\hline 37 & $>30$ & - & - & - & - \\
\hline 38 & $>30$ & - & - & - & - \\
\hline 39 & $>30$ & - & - & - & - \\
\hline 40 & 5.36 & $4.035-6.865$ & $\mathrm{Y}=-0.87+1.19 \mathrm{X}$ & 0.952 & 2.705 \\
\hline 41 & $>30$ & - & - & - & - \\
\hline 42 & $>30$ & - & - & - & - \\
\hline 43 & $>30$ & - & - & - & - \\
\hline 44 & $>30$ & - & - & - & - \\
\hline 45 & 0.75 & $0.011-1.661$ & $\mathrm{Y}=0.06+0.46 \mathrm{X}$ & 0.984 & 0.118 \\
\hline 46 & $>30$ & - & - & - & - \\
\hline 47 & $>30$ & - & - & - & - \\
\hline
\end{tabular}




\begin{tabular}{|c|c|c|c|c|c|}
\hline \multirow[b]{2}{*}{ compound } & \multicolumn{5}{|c|}{ Botrytis cinerea } \\
\hline & $\begin{array}{c}\mathrm{EC}_{50} \\
(\mu \mathrm{g} / \mathrm{mL})\end{array}$ & $95 \% \mathrm{CI}$ & Regression equation & $\mathrm{R}^{2}$ & $\begin{array}{c}\text { Chi-square } \\
\text { value }\end{array}$ \\
\hline 1 & 4.44 & $3.474-5.439$ & $Y=-1.01+1.56 \mathrm{X}$ & 0.968 & 4.654 \\
\hline 2 & 0.21 & $0.171-0.261$ & $\mathrm{Y}=1.35+2.03 \mathrm{X}$ & 0.977 & 3.575 \\
\hline 3 & 0.20 & $0.037-0.412$ & $Y=1.06+1.56 X$ & 0.948 & 3.898 \\
\hline 4 & 0.23 & $0.152-0.319$ & $Y=0.66+1.04 X$ & 0.983 & 1.014 \\
\hline 5 & 0.08 & $0.001-0.240$ & $Y=1.23+1.1 X$ & 0.919 & 4.245 \\
\hline 6 & 0.10 & $0.054-0.168$ & $\mathrm{Y}=0.93+0.95 \mathrm{X}$ & 0.997 & 0.132 \\
\hline 7 & 0.55 & $0.011-1.061$ & $Y=0.47+1.76 X$ & 0.948 & 5.131 \\
\hline 8 & 0.54 & $0.289-0.784$ & $\mathrm{Y}=0.27+0.98 \mathrm{X}$ & 0.919 & 2.843 \\
\hline 9 & 0.92 & $0.370-1.445$ & $\mathrm{Y}=0.03+0.9 \mathrm{X}$ & 0.975 & 0.610 \\
\hline 10 & 0.20 & $0.115-0.306$ & $Y=0.58+0.84 X$ & 0.981 & 0.804 \\
\hline 11 & 0.27 & $0.162-0.400$ & $Y=0.46+0.82 X$ & 0.992 & 0.341 \\
\hline 12 & 1.78 & $1.117-2.426$ & $Y=-0.25+0.99 X$ & 0.897 & 3.698 \\
\hline 13 & $>30$ & - & - & - & - \\
\hline 14 & 0.72 & $0.132-4.556$ & $Y=0.25+1.65 X$ & 0.943 & 9.115 \\
\hline 15 & 4.13 & $3.256-5.430$ & $Y=-0.76+1.23 X$ & 0.987 & 0.693 \\
\hline 16 & 0.28 & $0.050-0.592$ & $Y=0.75+1.35 X$ & 0.949 & 3.889 \\
\hline 17 & 0.76 & $0.357-1.167$ & $Y=0.14+1.08 X$ & 0.980 & 0.677 \\
\hline 18 & 0.29 & $0.191-0.411$ & $\mathrm{Y}=0.5+0.93 \mathrm{X}$ & 0.969 & 1.770 \\
\hline 19 & 0.78 & $0.441-1.113$ & $\mathrm{Y}=0.14+1.3 \mathrm{X}$ & 0.972 & 1.450 \\
\hline 20 & 0.90 & $0.472-1.315$ & $Y=0.06+1.06 X$ & 0.955 & 1.792 \\
\hline 21 & 0.09 & $0.056-0.140$ & $Y=1.37+1.42 X$ & 0.921 & 3.322 \\
\hline 22 & 0.11 & $0.050-0.179$ & $\mathrm{Y}=0.83+0.88 \mathrm{X}$ & 0.976 & 0.894 \\
\hline 23 & 0.13 & $0.104-0.175$ & $\mathrm{Y}=1.09+1.27 \mathrm{X}$ & 0.966 & 3.624 \\
\hline 24 & 0.07 & $0.034-0.471$ & $Y=2.08+2.05 X$ & 0.958 & 9.148 \\
\hline 25 & 0.12 & $0.001-0.553$ & $Y=1.26+1.44 X$ & 0.929 & 9.439 \\
\hline 26 & 2.41 & $1.946-2.903$ & $Y=-0.62+1.63 X$ & 0.971 & 2.500 \\
\hline 27 & 3.92 & $3.237-4.826$ & $Y=-0.93+1.56 X$ & 0.973 & 2.231 \\
\hline 28 & 5.00 & $3.356-6.538$ & $\mathrm{Y}=-1.07+1.53 \mathrm{X}$ & 0.987 & 0.924 \\
\hline 29 & 8.67 & $1.113-18.105$ & $\mathrm{Y}=-2.07+2.23 \mathrm{X}$ & 0.935 & 8.008 \\
\hline 30 & $>30$ & - & - & - & - \\
\hline 31 & 5.19 & $4.234-6.205$ & $\mathrm{Y}=-1.25+1.75 \mathrm{X}$ & 0.995 & 0.438 \\
\hline 32 & $>30$ & - & - & - & - \\
\hline 33 & $>30$ & - & - & - & - \\
\hline
\end{tabular}




\begin{tabular}{lllcll}
34 & 3.93 & $0.263-7.786$ & $\mathrm{Y}=-1.17+2 \mathrm{X}$ & 0.924 & 7.146 \\
35 & 0.16 & $0.115-0.208$ & $\mathrm{Y}=1.26+1.59 \mathrm{X}$ & 0.976 & 2.029 \\
36 & 0.16 & $0.111-0.215$ & $\mathrm{Y}=1.1+1.4 \mathrm{X}$ & 0.983 & 1.262 \\
37 & 0.28 & $0.047-0.621$ & $\mathrm{Y}=0.79+1.43 \mathrm{X}$ & 0.954 & 4.565 \\
38 & 0.49 & $0.001-0.995$ & $\mathrm{Y}=0.5+1.56 \mathrm{X}$ & 0.918 & 4.695 \\
39 & 0.42 & $0.001-2.389$ & $\mathrm{Y}=0.5+1.28 \mathrm{X}$ & 0.912 & 7.189 \\
40 & 0.14 & $0.096-0.194$ & $\mathrm{Y}=1.18+1.42 \mathrm{X}$ & 0.970 & 1.714 \\
41 & 0.21 & $0.144-0.284$ & $\mathrm{Y}=0.83+1.24 \mathrm{X}$ & 0.950 & 3.465 \\
42 & 0.13 & $0.091-0.182$ & $\mathrm{Y}=1.2+1.36 \mathrm{X}$ & 0.968 & 1.840 \\
43 & 0.17 & $0.001-0.496$ & $\mathrm{Y}=0.85+1.1 \mathrm{X}$ & 0.915 & 5.814 \\
44 & 0.19 & $0.007-0.470$ & $\mathrm{Y}=0.77+1.08 \mathrm{X}$ & 0.939 & 4.023 \\
45 & 0.10 & $0.053-0.157$ & $\mathrm{Y}=1+0.99 \mathrm{X}$ & 0.973 & 1.099 \\
46 & 0.11 & $0.059-0.181$ & $\mathrm{Y}=0.86+0.9 \mathrm{X}$ & 0.943 & 2.536 \\
47 & 0.09 & $0.003-0.258$ & $\mathrm{Y}=1.03+1.02 \mathrm{X}$ & 0.942 & 4.192 \\
48 & 0.17 & $0.005-0.423$ & $\mathrm{Y}=0.87+1.13 \mathrm{X}$ & 0.937 & 4.376 \\
49 & 0.23 & $0.148-0.323$ & $\mathrm{Y}=0.63+0.99 \mathrm{X}$ & 0.985 & 0.791 \\
\hline
\end{tabular}

\begin{tabular}{|c|c|c|c|c|c|}
\hline \multirow[b]{2}{*}{ compound } & \multicolumn{5}{|c|}{ Fusarium graminearum } \\
\hline & $\begin{array}{c}\mathrm{EC}_{50} \\
(\mu \mathrm{g} / \mathrm{mL})\end{array}$ & $95 \% \mathrm{CI}$ & Regression equation & $\mathrm{R}^{2}$ & $\begin{array}{c}\text { Chi-square } \\
\text { value }\end{array}$ \\
\hline 1 & 16.31 & $13.957-19.281$ & $\mathrm{Y}=-2.27+1.87 \mathrm{X}$ & 0.998 & 0.238 \\
\hline 2 & 1.83 & $1.462-2.204$ & $Y=-0.46+1.76 X$ & 0.990 & 0.799 \\
\hline 3 & 2.77 & $0.930-6.527$ & $Y=-0.66+1.5 X$ & 0.948 & 4.213 \\
\hline 4 & 13.95 & $6.492-26.433$ & $Y=-2.04+1.78 X$ & 0.960 & 4.058 \\
\hline 5 & 0.94 & $0.482-1.568$ & $Y=-0.03+0.98 X$ & 0.908 & 2.762 \\
\hline 6 & 3.77 & $2.016-5.446$ & $Y=-0.76+1.29 X$ & 0.983 & 0.848 \\
\hline 7 & 5.31 & $3.510-7.015$ & $Y=-1.02+1.41 X$ & 0.986 & 0.937 \\
\hline 8 & 12.82 & $10.731-15.140$ & $Y=-2.06+1.85 X$ & 0.976 & 2.440 \\
\hline 9 & 16.79 & $11.124-26.010$ & $Y=-0.92+0.75 X$ & 0.982 & 0.380 \\
\hline 10 & 8.47 & $6.030-10.900$ & $\mathrm{Y}=-1.14+0.925 \mathrm{X}$ & 0.925 & 3.639 \\
\hline 11 & 20.87 & $15.333-30.564$ & $Y=-1.24+0.94 X$ & 0.950 & 1.619 \\
\hline 12 & 22.34 & $12.041-58.218$ & $Y=-2.68+1.98 X$ & 0.962 & 5.054 \\
\hline 13 & 22.67 & $19.289-27.128$ & $Y=-2.65+1.95 X$ & 0.972 & 3.784 \\
\hline 14 & 8.27 & $2.077-14.785$ & $Y=-1.75+1.92 X$ & 0.945 & 5.039 \\
\hline 15 & $>30$ & - & - & - & - \\
\hline 16 & 4.86 & $3.673-5.943$ & $Y=-1.58+2.27 X$ & 0.998 & 0.079 \\
\hline 17 & 5.70 & $4.268-7.048$ & $Y=-1.34+1.8 X$ & 0.955 & 2.688 \\
\hline 18 & 8.75 & $6.863-10.664$ & $Y=-1.51+1.61 X$ & 0.954 & 3.374 \\
\hline
\end{tabular}




\begin{tabular}{|c|c|c|c|c|c|}
\hline 19 & 5.24 & $0.545-9.948$ & $\mathrm{Y}=-0.36+0.5 \mathrm{X}$ & 0.979 & 0.189 \\
\hline 20 & 1.88 & $0.001-6.428$ & $Y=-0.09+0.33 X$ & 0.978 & 0.086 \\
\hline 21 & 2.80 & $0.176-5.358$ & $Y=-0.99+2.14 X$ & 0.946 & 5.314 \\
\hline 22 & 8.63 & $7.095-10.186$ & $Y=-1.83+1.96 X$ & 0.960 & 3.511 \\
\hline 23 & 6.62 & $4.277-8.846$ & $\mathrm{Y}=-1.02+1.24 \mathrm{X}$ & 0.936 & 3.041 \\
\hline 24 & 1.92 & $0.830-3.002$ & $Y=-0.18+0.65 X$ & 0.967 & 0.472 \\
\hline 25 & 6.00 & $3.837-8.048$ & $Y=-0.94+1.22 X$ & 0.967 & 1.414 \\
\hline 26 & 9.44 & $8.033-10.910$ & $\mathrm{Y}=-2.2+2.26 \mathrm{X}$ & 0.971 & 3.069 \\
\hline 27 & 18.65 & $14.782-24.042$ & $\mathrm{Y}=-1.63+1.28 \mathrm{X}$ & 0.971 & 1.686 \\
\hline 28 & $>30$ & - & - & - & - \\
\hline 29 & $>30$ & - & - & - & - \\
\hline 30 & $>30$ & - & - & - & - \\
\hline 31 & $>30$ & - & - & - & - \\
\hline 32 & $>30$ & - & - & - & - \\
\hline 33 & $>30$ & - & - & - & - \\
\hline 34 & $>30$ & - & - & - & - \\
\hline 35 & 1.63 & $1.114-2.136$ & $Y=-0.25+1.18 X$ & 0.987 & 0.608 \\
\hline 36 & 1.72 & $0.001-4.399$ & $\mathrm{Y}=-0.31+1.3 \mathrm{X}$ & 0.912 & 5.581 \\
\hline 37 & 2.82 & $2.285-3.456$ & $Y=-0.68+1.5 X$ & 0.996 & 0.322 \\
\hline 38 & 3.46 & $2.947-4.100$ & $Y=-1.03+1.91 X$ & 0.980 & 2.416 \\
\hline 39 & 4.22 & $2.005-14.756$ & $\mathrm{Y}=-1.01+1.6 \mathrm{X}$ & 0.952 & 4.426 \\
\hline 40 & 1.48 & $0.762-2.164$ & $Y=-0.14+0.82 X$ & 0.948 & 1.342 \\
\hline 41 & 1.79 & $0.053-3.924$ & $Y=-0.34+1.32 X$ & 0.935 & 4.104 \\
\hline 42 & 1.19 & $0.526-1.817$ & $Y=-0.06+0.83 X$ & 0.972 & 0.646 \\
\hline 43 & 2.05 & $1.480-2.624$ & $Y=-0.39+1.22 X$ & 0.931 & 3.544 \\
\hline 44 & 2.82 & $2.177-3.571$ & $\mathrm{Y}=-0.57+1.27 \mathrm{X}$ & 0.989 & 0.586 \\
\hline 45 & 6.51 & 4.963-9.614 & $Y=-0.99+1.22 X$ & 0.967 & 1.517 \\
\hline 46 & 9.49 & $6.643-17.455$ & $Y=-1.03+1.06 \mathrm{X}$ & 0.987 & 0.427 \\
\hline 47 & 19.50 & $10.431-88.468$ & $\mathrm{Y}=-1.02+0.8 \mathrm{X}$ & 0.926 & 1.569 \\
\hline 48 & 1.22 & $0690-1.716$ & $\mathrm{Y}=0.09+1.07 \mathrm{X}$ & 0.985 & 0.549 \\
\hline 49 & 6.36 & 4.974-8.922 & $\mathrm{Y}=-1.07+1.33 \mathrm{X}$ & 0.997 & 0.167 \\
\hline
\end{tabular}

\begin{tabular}{cccccc}
\hline & \multicolumn{5}{c}{ Magnaporthe oryzae } \\
\cline { 2 - 6 } compound & $\begin{array}{c}\mathrm{EC}_{50} \\
(\mu \mathrm{g} / \mathrm{mL})\end{array}$ & $95 \% \mathrm{CI}$ & Regression equation & $\mathrm{R}^{2}$ & $\begin{array}{c}\text { Chi-square } \\
\text { value }\end{array}$ \\
\hline 1 & 17.63 & $13.821-23.513$ & $\mathrm{Y}=-1.39+1.11 \mathrm{X}$ & 0.998 & 0.165 \\
2 & 0.81 & $0.474-1.135$ & $\mathrm{Y}=0.08+0.89 \mathrm{X}$ & 0.915 & 2.459 \\
3 & 1.30 & $0.117-5.119$ & $\mathrm{Y}=-0.2+1.57 \mathrm{X}$ & 0.928 & 6.082
\end{tabular}




\begin{tabular}{|c|c|c|c|c|c|}
\hline 4 & 17.37 & $13.989-21.808$ & $Y=-1.71+1.38 X$ & 0.946 & 3.611 \\
\hline 5 & 0.63 & $0.001-1.721$ & $\mathrm{Y}=0.07+0.36 \mathrm{X}$ & 0.954 & 0.209 \\
\hline 6 & 0.82 & $0.249-1.387$ & $\mathrm{Y}=0.07+0.79 \mathrm{X}$ & 0.995 & 0.098 \\
\hline 7 & 8.54 & $6.465-10.615$ & $Y=-1.41+1.52 X$ & 0.979 & 1.391 \\
\hline 8 & 11.41 & $8.408-14.685$ & $\mathrm{Y}=-1.23+1.16 \mathrm{X}$ & 0.972 & 1.280 \\
\hline 9 & 17.98 & $4.955-136.423$ & $Y=-1.58+1.26 X$ & 0.925 & 4.394 \\
\hline 10 & 10.19 & $1.354-22.822$ & $Y=-1.83+1.82 X$ & 0.944 & 6.625 \\
\hline 11 & 10.02 & $6.658-13.514$ & $\mathrm{Y}=-0.99+0.99 \mathrm{X}$ & 0.993 & 0.257 \\
\hline 12 & 20.41 & $4.240-287.796$ & $\mathrm{Y}=-3.4+2.58 \mathrm{X}$ & 0.934 & 13.498 \\
\hline 13 & 15.90 & $8.193-31.374$ & $Y=-2.26+1.89 X$ & 0.963 & 4.289 \\
\hline 14 & 6.07 & $0.001-12.539$ & $Y=-1.79+2.29 X$ & 0.955 & 7.969 \\
\hline 15 & 16.57 & $12.523-22.107$ & $\mathrm{Y}=-1.32+1.08 \mathrm{X}$ & 0.933 & 2.910 \\
\hline 16 & 3.24 & $2.420-4.058$ & $\mathrm{Y}=-0.81+1.57 \mathrm{X}$ & 0.978 & 1.836 \\
\hline 17 & 5.92 & $3.517-8.199$ & $\mathrm{Y}=-0.84+1.09 \mathrm{X}$ & 0.915 & 3.598 \\
\hline 18 & 18.73 & $7.668-62.084$ & $\mathrm{Y}=-2.73+2.14 \mathrm{X}$ & 0.940 & 8.013 \\
\hline 19 & 4.96 & $2.961-7.257$ & $Y=-0.53+0.76 X$ & 0.894 & 2.727 \\
\hline 20 & 2.06 & $1.605-2.533$ & $\mathrm{Y}=-0.48+1.52 \mathrm{X}$ & 0.974 & 1.920 \\
\hline 21 & 3.14 & $2.305-3.958$ & $\mathrm{Y}=-0.72+1.46 \mathrm{X}$ & 0.957 & 3.484 \\
\hline 22 & 5.20 & $0.326-10.233$ & $\mathrm{Y}=-0.33+0.47 \mathrm{X}$ & 0.951 & 0.397 \\
\hline 23 & 11.96 & $4.251-23.352$ & $Y=-1.74+1.62 X$ & 0.955 & 3.835 \\
\hline 24 & 2.82 & $1.505-4.044$ & $\mathrm{Y}=-0.45+0.99 \mathrm{X}$ & 0.982 & 0.614 \\
\hline 25 & 5.09 & $0.044-9.986$ & $\mathrm{Y}=-1.22+1.76 \mathrm{X}$ & 0.923 & 5.080 \\
\hline 26 & 12.67 & $9.790-15.957$ & $\mathrm{Y}=-1.41+1.28 \mathrm{X}$ & 0.981 & 1.073 \\
\hline 27 & $>30$ & - & - & - & - \\
\hline 28 & $>30$ & - & - & - & - \\
\hline 29 & 9.71 & $0.752-20.342$ & $\mathrm{Y}=-0.4+0.4 \mathrm{X}$ & 0.963 & 0.232 \\
\hline 30 & $>30$ & - & - & - & - \\
\hline 31 & $>30$ & - & - & - & - \\
\hline 32 & $>30$ & - & - & - & - \\
\hline 33 & $>30$ & - & - & - & - \\
\hline 34 & $>30$ & - & - & - & - \\
\hline 35 & 0.64 & $0.166-2.370$ & $\mathrm{Y}=0.3+1.52 \mathrm{X}$ & 0.950 & 6.505 \\
\hline 36 & 0.97 & $0.780-1.177$ & $\mathrm{Y}=0.01+1.63 \mathrm{X}$ & 0.974 & 2.227 \\
\hline 37 & 1.05 & $0.698-1.439$ & $\mathrm{Y}=-0.02+0.94 \mathrm{X}$ & 0.944 & 1.796 \\
\hline 38 & 1.19 & $0.003-5.483$ & $\mathrm{Y}=-0.12+1.47 \mathrm{X}$ & 0.925 & 5.969 \\
\hline 39 & 1.84 & $1.324-2.379$ & $Y=-0.32+1.21 \mathrm{X}$ & 0.988 & 0.655 \\
\hline 40 & 0.62 & $0.296-1.195$ & $\mathrm{Y}=0.39+1.81 \mathrm{X}$ & 0.975 & 4.310 \\
\hline 41 & 0.69 & $0.576-0.842$ & $\mathrm{Y}=0.27+1.67 \mathrm{X}$ & 0.993 & 1.102 \\
\hline 42 & 0.83 & $0.276-4.056$ & $\mathrm{Y}=0.12+1.46 \mathrm{X}$ & 0.950 & 6.305 \\
\hline 43 & 0.79 & $0.664-0.959$ & $\mathrm{Y}=0.19+1.74 \mathrm{X}$ & 0.980 & 3.303 \\
\hline 44 & 1.10 & $0.052-2.766$ & $Y=-0.06+1.23 X$ & 0.927 & 4.073 \\
\hline 45 & 0.62 & $0.001-1.748$ & $\mathrm{Y}=0.07+0.34 \mathrm{X}$ & 0.905 & 0.423 \\
\hline 46 & 6.55 & $3.043-9.854$ & $\mathrm{Y}=-0.65+0.8 \mathrm{X}$ & 0.973 & 0.655 \\
\hline 47 & 10.59 & $4.678-17.167$ & $\mathrm{Y}=-0.61+0.6 \mathrm{X}$ & 0.984 & 0.204 \\
\hline
\end{tabular}




\begin{tabular}{llllll}
48 & 0.94 & $0.765-1.124$ & $\mathrm{Y}=0.04+1.85 \mathrm{X}$ & 0.969 & 3.728 \\
49 & 0.78 & $0.056-1.586$ & $\mathrm{Y}=0.06+0.54 \mathrm{X}$ & 0.973 & 0.264 \\
\hline
\end{tabular}

\begin{tabular}{|c|c|c|c|c|c|}
\hline \multirow[b]{2}{*}{ compound } & \multicolumn{5}{|c|}{ Mycosphaerlla melonis } \\
\hline & $\begin{array}{c}\mathrm{EC}_{50} \\
(\mu \mathrm{g} / \mathrm{mL})\end{array}$ & $95 \% \mathrm{CI}$ & Regression equation & $\mathrm{R}^{2}$ & $\begin{array}{c}\text { Chi-square } \\
\text { value }\end{array}$ \\
\hline 1 & 1.16 & $0.542-1.814$ & $Y=-0.2+1.45 X$ & 0.960 & 1.431 \\
\hline 2 & 1.77 & $0.170-3.969$ & $Y=-0.83+3.52 X$ & 0.966 & 10.584 \\
\hline 3 & 0.78 & $0.207-1.347$ & $Y=0.2+1.94 X$ & 0.952 & 3.839 \\
\hline 4 & 1.87 & $1.534-2.214$ & $Y=-0.55+2.01 X$ & 0.998 & 0.182 \\
\hline 5 & 0.98 & $0.752-1.196$ & $Y=-0.03+2.61 X$ & 0.991 & 1.069 \\
\hline 6 & 0.98 & $0.739-1.201$ & $\mathrm{Y}=0.05+2.2 \mathrm{X}$ & 0.987 & 0.566 \\
\hline 7 & 1.01 & $0.688-1.327$ & $Y=0.01+1.56 X$ & 0.961 & 1.808 \\
\hline 8 & 2.22 & $0.001-4.809$ & $\mathrm{Y}=-0.43+1.39 \mathrm{X}$ & 0.902 & 4.885 \\
\hline 9 & 2.17 & $1.748-2.616$ & $Y=-0.57+1.7 X$ & 0.990 & 0.943 \\
\hline 10 & 0.88 & $0.571-1.181$ & $Y=0.09+0.59 X$ & 0.997 & 0.134 \\
\hline 11 & 1.56 & $0.001-3.741$ & $\mathrm{Y}=-0.38+2.07 \mathrm{X}$ & 0.925 & 9.582 \\
\hline 12 & 7.29 & $1.527-12.831$ & $Y=-2.13+2.45 \mathrm{X}$ & 0.952 & 5.238 \\
\hline 13 & 7.39 & $6.043-8.717$ & $Y=-1.98+2.28 \mathrm{X}$ & 0.982 & 2.428 \\
\hline 14 & 2.91 & $2.219-3.544$ & $Y=-1+2.1 X$ & 0.985 & 1.050 \\
\hline 15 & 4.32 & $1.567-6.997$ & $Y=-0.5+0.79 X$ & 0.908 & 2.154 \\
\hline 16 & 1.36 & $1.054-1.669$ & $\mathrm{Y}=-0.24+1.78 \mathrm{X}$ & 0.992 & 0.642 \\
\hline 17 & 3.31 & $2.244-4.324$ & $Y=-0.66+1.27 \mathrm{X}$ & 0.983 & 0.878 \\
\hline 18 & 2.01 & $1.522-2.501$ & $\mathrm{Y}=-0.43+1.42 \mathrm{X}$ & 0.996 & 0.274 \\
\hline 19 & 1.17 & $0.003-2.390$ & $Y=-0.11+1.69 X$ & 0.946 & 5.499 \\
\hline 20 & 1.63 & $1.363-1.916$ & $Y=-0.46+2.2 X$ & 0.977 & 3.441 \\
\hline 21 & 0.83 & $0.453-1.197$ & $\mathrm{Y}=0.07+0.83 \mathrm{X}$ & 0.945 & 1.376 \\
\hline 22 & 1.16 & $0.835-1.464$ & $Y=-0.1+1.7 X$ & 0.988 & 0.690 \\
\hline 23 & 0.14 & $0.037-0.284$ & $\mathrm{Y}=0.42+0.5 \mathrm{X}$ & 0.929 & 1.700 \\
\hline 24 & 0.11 & $0.012-0.269$ & $\mathrm{Y}=1.11+1.3 \mathrm{X}$ & 0.916 & 7.395 \\
\hline 25 & 0.14 & $0.082-0.226$ & $\mathrm{Y}=0.77+0.97 \mathrm{X}$ & 0.930 & 4.764 \\
\hline 26 & 4.06 & $2.507-5.521$ & $\mathrm{Y}=-0.93+1.51 \mathrm{X}$ & 0.997 & 0.205 \\
\hline 27 & 7.60 & $5.309-9.829$ & $\mathrm{Y}=-1.11+1.26 \mathrm{X}$ & 0.969 & 1.539 \\
\hline 28 & 2.12 & $1.573-2.678$ & $Y=-0.42+1.3 X$ & 0.991 & 0.518 \\
\hline 29 & 0.78 & $0.027-2.250$ & $\mathrm{Y}=0.06+0.75 \mathrm{X}$ & 0.968 & 0.532 \\
\hline 30 & 0.45 & $0.016-1.074$ & $Y=0.19+0.52 X$ & 0.849 & 1.681 \\
\hline 31 & 11.71 & $4.816-21.214$ & $Y=-1.91+1.79 X$ & 0.956 & 4.161 \\
\hline 32 & $>30$ & - & - & - & - \\
\hline
\end{tabular}




\begin{tabular}{cccccc}
33 & $>30$ & - & - & - & - \\
34 & 2.58 & $0.476-6.126$ & $\mathrm{Y}=-0.71+1.76 \mathrm{X}$ & 0.940 & 6.181 \\
35 & 0.43 & $0.217-0.760$ & $\mathrm{Y}=0.2+0.54 \mathrm{X}$ & 0.902 & 2.022 \\
36 & 0.50 & $0.280-0.832$ & $\mathrm{Y}=0.18+0.59 \mathrm{X}$ & 0.975 & 0.562 \\
37 & 0.75 & $0.555-0.957$ & $\mathrm{Y}=0.17+1.44 \mathrm{X}$ & 0.998 & 0.145 \\
38 & 0.96 & $0.580-1.323$ & $\mathrm{Y}=0.03+1.33 \mathrm{X}$ & 0.999 & 0.054 \\
39 & 0.77 & $0.331-1.205$ & $\mathrm{Y}=0.1+0.98 \mathrm{X}$ & 0.960 & 1.230 \\
40 & 0.30 & $0.004-0.934$ & $\mathrm{Y}=0.67+1.28 \mathrm{X}$ & 0.936 & 6.373 \\
41 & 0.24 & $0.157-0.333$ & $\mathrm{Y}=0.64+1.04 \mathrm{X}$ & 0.964 & 2.379 \\
42 & 0.50 & $0.322-0.684$ & $\mathrm{Y}=0.39+1.36 \mathrm{X}$ & 0.991 & 0.433 \\
43 & 0.52 & $0.334-0.711$ & $\mathrm{Y}=0.36+1.28 \mathrm{X}$ & 0.970 & 1.235 \\
44 & 0.71 & $0.440-0.984$ & $\mathrm{Y}=0.15+1.06 \mathrm{X}$ & 0.950 & 1.894 \\
45 & 0.18 & $0.001-0.490$ & $\mathrm{Y}=0.86+1.17 \mathrm{X}$ & 0.942 & 5.095 \\
46 & 0.28 & $0.121-0.485$ & $\mathrm{Y}=0.3+0.56 \mathrm{X}$ & 0.994 & 0.113 \\
47 & 1.22 & $0.532-1.873$ & $\mathrm{Y}=-0.08+0.83 \mathrm{X}$ & 0.970 & 0.680 \\
48 & 0.64 & $0.001-1.391$ & $\mathrm{Y}=0.32+1.6 \mathrm{X}$ & 0.927 & 6.151 \\
49 & 0.87 & $0.496-1.233$ & $\mathrm{Y}=0.09+1.25 \mathrm{X}$ & 0.962 & 1.713 \\
\hline
\end{tabular}

3. Physical properties of the target compounds.<smiles>Cn1c2nc3ccccc3c-2cc2ccccc21</smiles>

5-methyl-5H-indolo[2,3-b]quinoline (Neocryptolepine, 1) 
Yield, 62\%; red solid, m.p. 105.32-106.54 ${ }^{\circ} \mathrm{C}$; ${ }^{1} \mathrm{H}$ NMR (400 MHz, DMSO- $\left.d_{6}\right) \delta 8.64$ (s, 1H), $8.03(\mathrm{~d}, J=7.5 \mathrm{~Hz}, 1 \mathrm{H}), 7.94(\mathrm{~d}, J=8.0 \mathrm{~Hz}, 1 \mathrm{H}), 7.73(\mathrm{~d}, J=8.5 \mathrm{~Hz}, 1 \mathrm{H})$, $7.68(\mathrm{~m}, 1 \mathrm{H}), 7.61(\mathrm{~d}, J=7.9 \mathrm{~Hz}, 1 \mathrm{H}), 7.48(\mathrm{t}, J=7.6 \mathrm{~Hz}, 1 \mathrm{H}), 7.36(\mathrm{~m}, 1 \mathrm{H}), 7.15(\mathrm{t}$, $J=7.4 \mathrm{~Hz}, 1 \mathrm{H}), 4.14(\mathrm{~s}, 3 \mathrm{H}) .{ }^{13} \mathrm{C} \mathrm{NMR}\left(101 \mathrm{MHz}, \mathrm{DMSO}-d_{6}\right) \delta 155.72,155.70$, $136.85,130.81,130.18,129.13,129.02,127.24,124.30,122.01,121.74,120.52$, 119.57, 117.61, 115.00, 32.96. MS-ESI $\mathrm{m} / \mathrm{z}$ calcd for $\mathrm{C}_{16} \mathrm{H}_{12} \mathrm{~N}_{2}[\mathrm{M}+\mathrm{H}]^{+}$: 233.1530; found: 233.1548 .

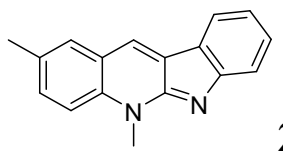

\section{2,5-dimethyl-5H-indolo[2,3-b]quinoline (2)}

Yield, 51\%; red solid, m.p. 123.83-124.43 ${ }^{\circ} \mathrm{C} ;{ }^{1} \mathrm{H}$ NMR (400 MHz, DMSO- $\left.d_{6}\right) \delta 8.81$ (s, 1H), $8.12(\mathrm{~d}, J=7.5 \mathrm{~Hz}, 1 \mathrm{H}), 7.88-7.81(\mathrm{~m}, 2 \mathrm{H}), 7.63(\mathrm{~d}, J=8.7 \mathrm{~Hz}, 1 \mathrm{H}), 7.57$ (d, $J=7.9 \mathrm{~Hz}, 1 \mathrm{H}), 7.47$ (t, $J=7.6 \mathrm{~Hz}, 1 \mathrm{H}), 7.16(\mathrm{t}, J=7.4 \mathrm{~Hz}, 1 \mathrm{H}), 4.26(\mathrm{~s}, 3 \mathrm{H})$, $2.47(\mathrm{~s}, 3 \mathrm{H}) .{ }^{13} \mathrm{C}$ NMR $\left(101 \mathrm{MHz}, \mathrm{DMSO}-d_{6}\right) \delta 155.77,155.72,135.27,132.49$ $131.36,129.59,129.08,129.01,127.31,124.30,121.79,120.65,119.42,117.51$, 115.19, 33.12, 20.78. MS-ESI m/z calcd for $\mathrm{C}_{17} \mathrm{H}_{14} \mathrm{~N}_{2}[\mathrm{M}+\mathrm{H}]^{+}$: 247.1657; found: 247.1715 .

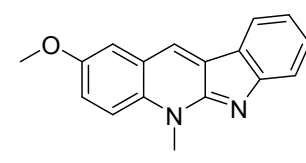

\section{2-methoxy-5-methyl-5H-indolo[2,3-b]quinoline (3)}

Yield, 43\%; red solid, m.p. 131.52-134.62 ${ }^{\circ} \mathrm{C} ;{ }^{1} \mathrm{H}$ NMR (400 MHz, DMSO- $\left.d_{6}\right) \delta 8.85$ (s, 1H), $8.11(\mathrm{~d}, J=7.5 \mathrm{~Hz}, 1 \mathrm{H}), 7.90(\mathrm{~d}, J=9.3 \mathrm{~Hz}, 1 \mathrm{H}), 7.62(\mathrm{~s}, 1 \mathrm{H}), 7.56$ (d, $J=$ $7.9 \mathrm{~Hz}, 1 \mathrm{H}), 7.49(\mathrm{~m}, 1 \mathrm{H}), 7.46(\mathrm{~d}, J=3.1 \mathrm{~Hz}, 1 \mathrm{H}), 7.15(\mathrm{t}, J=7.4 \mathrm{~Hz}, 1 \mathrm{H}), 4.28(\mathrm{~s}$, 3H), 3.90 (s, 3H). ${ }^{13} \mathrm{C}$ NMR (101 MHz, DMSO-d $\left.d_{6}\right) \delta 155.96,155.48,154.45,131.96$, $129.18,128.67,127.70,124.02,121.78,121.46,120.70,119.23,117.43,116.71$, 
110.82, 56.03, 33.28. MS-ESI $\mathrm{m} / \mathrm{z}$ calcd for $\mathrm{C}_{17} \mathrm{H}_{14} \mathrm{~N}_{2} \mathrm{O}[\mathrm{M}+\mathrm{H}]^{+}:$: 263.1106; found: 263.1676 .

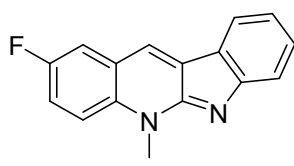

\section{2-fluoro-5-methyl-5H-indolo[2,3-b]quinoline (4)}

Yield, 70\%; red solid, m.p. $143.46-144.16{ }^{\circ} \mathrm{C} ;{ }^{1} \mathrm{H}$ NMR (400 MHz, DMSO- $\left.d_{6}\right) \delta 8.85$ $(\mathrm{s}, 1 \mathrm{H}), 8.10(\mathrm{~d}, J=7.6 \mathrm{~Hz}, 1 \mathrm{H}), 7.97(\mathrm{~m}, 2 \mathrm{H}), 7.71(\mathrm{~m}, 1 \mathrm{H}), 7.58(\mathrm{~d}, J=7.9 \mathrm{~Hz}, 1 \mathrm{H})$, $7.50(\mathrm{t}, J=7.6 \mathrm{~Hz}, 1 \mathrm{H}), 7.18(\mathrm{t}, J=7.4 \mathrm{~Hz}, 1 \mathrm{H}), 4.28(\mathrm{~s}, 3 \mathrm{H}) .{ }^{13} \mathrm{C}$ NMR $(101 \mathrm{MHz}$, DMSO- $\left.d_{6}\right) \delta 158.39,156.04,155.68,133.82,129.61,128.51,128.29(\mathrm{~d}, J=3.6 \mathrm{~Hz})$, 123.89, 121.99, $121.33(\mathrm{~d}, J=9.0 \mathrm{~Hz}), 119.72,119.07$ (d, $J=24.5 \mathrm{~Hz}), 117.63$, $117.46(\mathrm{~d}, J=8.5 \mathrm{~Hz}), 114.33(\mathrm{~d}, J=22.4 \mathrm{~Hz}), 33.45$. MS-ESI $\mathrm{m} / \mathrm{z}$ : calcd for $\mathrm{C}_{16} \mathrm{H}_{11} \mathrm{FN}_{2}[\mathrm{M}+\mathrm{H}]^{+}:$251.1510; found: 251.1539 .

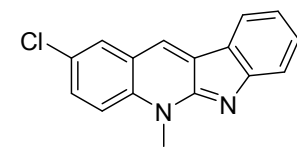

\section{2-chloro-5-methyl-5H-indolo[2,3-b]quinoline (5)}

Yield, 50\%; red solid, m.p. 166.28-166.78 ${ }^{\circ} \mathrm{C} ;{ }^{1} \mathrm{H}$ NMR (400 MHz, DMSO- $\left.d_{6}\right) \delta 8.83$ (s, 1H), $8.19(\mathrm{~d}, J=2.6 \mathrm{~Hz}, 1 \mathrm{H}), 8.10(\mathrm{~d}, J=7.5 \mathrm{~Hz}, 1 \mathrm{H}), 7.96(\mathrm{~d}, J=9.0 \mathrm{~Hz}, 1 \mathrm{H})$, $7.81(\mathrm{~d}, J=6.7 \mathrm{~Hz}, 1 \mathrm{H}), 7.59(\mathrm{~d}, J=7.9 \mathrm{~Hz}, 1 \mathrm{H}), 7.50(\mathrm{t}, J=7.6 \mathrm{~Hz}, 1 \mathrm{H}), 7.19(\mathrm{t}, J=$ $7.4 \mathrm{~Hz}, 1 \mathrm{H}), 4.26(\mathrm{~s}, 3 \mathrm{H}) .{ }^{13} \mathrm{C}$ NMR $\left(101 \mathrm{MHz}, \mathrm{DMSO}-d_{6}\right) \delta 155.93,155.62,135.66$, $130.57,129.66,128.80,128.45,128.06,126.21,124.09,122.00,121.71,119.94$, 117.78, 117.41, 33.37. MS-ESI m/z: calcd for $\mathrm{C}_{16} \mathrm{H}_{11} \mathrm{ClN}_{2}[\mathrm{M}+\mathrm{H}]^{+}$: 267.1301; found: 267.1306.

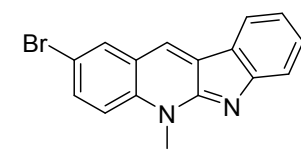

2-bromo-5-methyl-5H-indolo[2,3-b]quinoline (6) 
Yield, 54\%; red solid, m.p. $136.81-138.25{ }^{\circ} \mathrm{C} ;{ }^{1} \mathrm{H}$ NMR (400 MHz, DMSO- $\left.d_{6}\right) \delta 8.83$ (s, 1H), $8.32(\mathrm{~d}, J=2.1 \mathrm{~Hz}, 1 \mathrm{H}), 8.09(\mathrm{~d}, J=7.6 \mathrm{~Hz}, 1 \mathrm{H}), 7.94-7.84(\mathrm{~m}, 2 \mathrm{H}), 7.59$ $(\mathrm{d}, J=7.9 \mathrm{~Hz}, 1 \mathrm{H}), 7.54-7.43(\mathrm{~m}, 1 \mathrm{H}), 7.19(\mathrm{t}, J=7.4 \mathrm{~Hz}, 1 \mathrm{H}), 4.26(\mathrm{~s}, 3 \mathrm{H}) .{ }^{13} \mathrm{C}$ NMR (101 MHz, DMSO- $\left.d_{6}\right) \delta 155.94,155.60,136.01,133.21,131.87,129.66$, $128.40,128.01,124.14,122.26,121.99,119.97,117.80,117.64,114.06,33.34$. MS-ESI m/z: calcd for $\mathrm{C}_{16} \mathrm{H}_{11} \mathrm{BrN}_{2}[\mathrm{M}+\mathrm{H}]^{+}:$311.0810; found: 311.0870 .

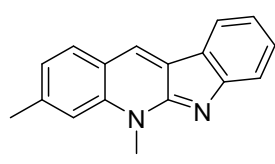

\section{3,5-dimethyl-5H-indolo[2,3-b]quinoline (7)}

Yield, 55\%; red solid, m.p. 172.19-174.69 ${ }^{\circ} \mathrm{C} ;{ }^{1} \mathrm{H}$ NMR (400 MHz, DMSO- $\left.d_{6}\right) \delta 8.87$ (s, 1H), $8.11(\mathrm{~d}, J=7.6 \mathrm{~Hz}, 1 \mathrm{H}), 8.01(\mathrm{~d}, J=8.1 \mathrm{~Hz}, 1 \mathrm{H}), 7.77(\mathrm{~s}, 1 \mathrm{H}), 7.58$ (d, $J=$ $7.9 \mathrm{~Hz}, 1 \mathrm{H}), 7.47$ (t, $J=7.6 \mathrm{~Hz}, 1 \mathrm{H}), 7.31(\mathrm{~d}, J=8.1 \mathrm{~Hz}, 1 \mathrm{H}), 7.17$ (t, $J=7.4 \mathrm{~Hz}, 1 \mathrm{H})$, $4.28(\mathrm{~s}, 3 \mathrm{H}), 2.59(\mathrm{~s}, 3 \mathrm{H}) .{ }^{13} \mathrm{C}$ NMR (101 MHz, DMSO- $\left.d_{6}\right) \delta 155.88,155.48,141.66$, $137.24,130.17,129.33,128.84,126.33,124.44,123.87,121.61,119.51,118.66$, 117.54, 115.11, 33.11, 22.43. MS-ESI m/z: calcd for $\mathrm{C}_{17} \mathrm{H}_{14} \mathrm{~N}_{2}[\mathrm{M}+\mathrm{H}]^{+}$: 247.1557; found: 247.1664 .

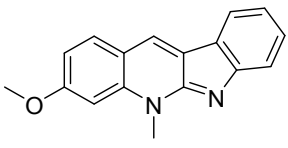

\section{3-methoxy-5-methyl-5H-indolo[2,3-b]quinoline (8)}

Yield, 50\%; brownish yellow solid, m.p. 238.14-240.14 ${ }^{\circ} \mathrm{C}$; ${ }^{1} \mathrm{H}$ NMR (400 MHz, DMSO- $\left.d_{6}\right) \delta 8.87(\mathrm{~s}, 1 \mathrm{H}), 8.08(\mathrm{~d}, J=7.6 \mathrm{~Hz}, 1 \mathrm{H}), 8.04(\mathrm{~d}, J=8.8 \mathrm{~Hz}, 1 \mathrm{H}), 7.57(\mathrm{~d}, J$ $=7.9 \mathrm{~Hz}, 1 \mathrm{H}), 7.45(\mathrm{t}, J=7.8 \mathrm{~Hz}, 1 \mathrm{H}), 7.32(\mathrm{~d}, J=2.3 \mathrm{~Hz}, 1 \mathrm{H}), 7.20-7.16(\mathrm{~m}, 1 \mathrm{H})$, $7.13(\mathrm{~d}, J=2.3 \mathrm{~Hz}, 1 \mathrm{H}), 4.27(\mathrm{~s}, 3 \mathrm{H}), 4.00(\mathrm{~s}, 3 \mathrm{H}) .{ }^{13} \mathrm{C}$ NMR $\left(101 \mathrm{MHz}, \mathrm{DMSO}-d_{6}\right) \delta$ $162.23,155.26,153.59,138.95,131.96,129.99,128.44,124.27,124.00,121.34$ 
$119.82,117.13,115.45,112.26,98.33,56.34,33.59$. MS-ESI $\mathrm{m} / \mathrm{z}$ : calcd for $\mathrm{C}_{17} \mathrm{H}_{14} \mathrm{~N}_{2} \mathrm{O}[\mathrm{M}+\mathrm{H}]^{+}:$: 263.1806; found: 263.1942 .

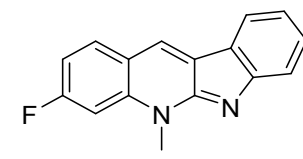

\section{3-fluoro-5-methyl-5H-indolo[2,3-b]quinoline (9)}

Yield, 62\%; red solid, m.p. 92.14-94.24 ${ }^{\circ} \mathrm{C} ;{ }^{1} \mathrm{H}$ NMR (400 MHz, DMSO- $d_{6}$ ) $\delta 8.93$ (s, 1H), $8.20(\mathrm{~d}, J=8.8 \mathrm{~Hz}, 1 \mathrm{H}), 8.12(\mathrm{~d}, J=7.6 \mathrm{~Hz}, 1 \mathrm{H}), 7.83(\mathrm{~d}, J=11.8 \mathrm{~Hz}, 1 \mathrm{H}), 7.59$ $(\mathrm{d}, J=7.9 \mathrm{~Hz}, 1 \mathrm{H}), 7.49(\mathrm{t}, J=7.6 \mathrm{~Hz}, 1 \mathrm{H}), 7.37(\mathrm{~m}, 1 \mathrm{H}), 7.19(\mathrm{t}, J=7.4 \mathrm{~Hz}, 1 \mathrm{H})$, $4.26(\mathrm{~s}, 3 \mathrm{H}) .{ }^{13} \mathrm{C}$ NMR $\left(101 \mathrm{MHz}, \mathrm{DMSO}-d_{6}\right) \delta 164.97,162.51,155.88,155.35$, $138.58(\mathrm{~d}, J=12.0 \mathrm{~Hz}), 132.79(\mathrm{~d}, J=10.7 \mathrm{~Hz}), 129.15,129.04,126.74,124.39$, 121.73, 119.96, $117.74(\mathrm{~d}, J=5.7 \mathrm{~Hz}), 110.82(\mathrm{~d}, J=23.8 \mathrm{~Hz}), 101.98(\mathrm{~d}, J=27.3$ $\mathrm{Hz}$ ), 33.51. MS-ESI m/z: calcd for $\mathrm{C}_{16} \mathrm{H}_{11} \mathrm{FN}_{2}[\mathrm{M}+\mathrm{H}]^{+}$: 251.1703; found: 251.1716.

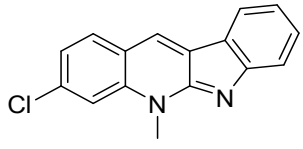

\section{3-chloro-5-methyl-5H-indolo[2,3-b]quinoline (10)}

Yield, 43\%; red solid, m.p. 181.13-183.33 ${ }^{\circ} \mathrm{C} ;{ }^{1} \mathrm{H}$ NMR (400 MHz, DMSO- $\left.d_{6}\right) \delta 8.94$ (s, 1H), $8.14(\mathrm{~d}, J=10.2 \mathrm{~Hz}, 2 \mathrm{H}), 8.05(\mathrm{~d}, J=1.9 \mathrm{~Hz}, 1 \mathrm{H}), 7.60(\mathrm{~d}, J=8.0 \mathrm{~Hz}, 1 \mathrm{H})$, $7.56-7.47(\mathrm{~m}, 2 \mathrm{H}), 7.20(\mathrm{t}, J=7.3 \mathrm{~Hz}, 1 \mathrm{H}), 4.29(\mathrm{~s}, 3 \mathrm{H}) .{ }^{13} \mathrm{C}$ NMR $(101 \mathrm{MHz}$, DMSO- $\left.d_{6}\right) \delta 155.69,155.65,137.82,135.71,131.94,129.45,128.84,127.69,124.29$, 122.52, 121.94, 120.06, 119.44, 117.82, 115.14, 33.41. MS-ESI m/z: calcd for $\mathrm{C}_{16} \mathrm{H}_{11} \mathrm{ClN}_{2}[\mathrm{M}+\mathrm{H}]^{+}:$267.1405; found: 267.1428 .

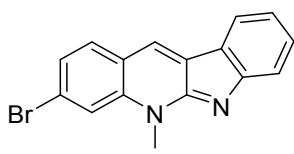

\section{3-bromo-5-methyl-5H-indolo[2,3-b]quinoline (11)}

Yield, 60\%; red solid, m.p. 197.86-198.66 ${ }^{\circ} \mathrm{C} ;{ }^{1} \mathrm{H}$ NMR (400 MHz, DMSO- $\left.d_{6}\right) \delta 8.90$ $(\mathrm{s}, 1 \mathrm{H}), 8.15(\mathrm{~d}, J=1.7 \mathrm{~Hz}, 1 \mathrm{H}), 8.12(\mathrm{~d}, J=7.6 \mathrm{~Hz}, 1 \mathrm{H}), 8.06(\mathrm{~d}, J=8.5 \mathrm{~Hz}, 1 \mathrm{H})$, $7.64(\mathrm{~d}, J=8.4 \mathrm{~Hz}, 1 \mathrm{H}), 7.59(\mathrm{~d}, J=7.9 \mathrm{~Hz}, 1 \mathrm{H}), 7.54-7.44(\mathrm{~m}, 1 \mathrm{H}), 7.19(\mathrm{t}, J=7.1$ 
$\mathrm{Hz}, 1 \mathrm{H}), 4.27$ (s, 3H). ${ }^{13} \mathrm{C}$ NMR (101 MHz, DMSO- $\left.d_{6}\right) \delta 155.71,155.60,137.91$, $131.93,129.45,128.83,127.80,125.23,124.49,124.30,121.93,120.05,119.65$, 117.96, 117.83, 33.36. MS-ESI m/z: calcd for $\mathrm{C}_{16} \mathrm{H}_{11} \mathrm{BrN}_{2}[\mathrm{M}+\mathrm{H}]^{+}$: 311.0826 ; found: 311.0833 .

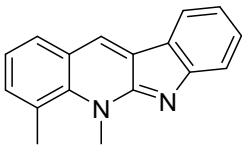

\section{4,5-dimethyl-5H-indolo[2,3-b]quinoline (12)}

Yield, 27\%; red solid, m.p. 128.75-130.85 ${ }^{\circ} \mathrm{C} ;{ }^{1} \mathrm{H}$ NMR (400 MHz, DMSO- $\left.d_{6}\right) \delta 8.84$ (s, 1H), 8.09 (d, $J=7.5 \mathrm{~Hz}, 1 \mathrm{H}), 7.93(\mathrm{~d}, J=8.0 \mathrm{~Hz}, 1 \mathrm{H}), 7.58(\mathrm{~d}, J=7.8 \mathrm{~Hz}, 2 \mathrm{H})$, $7.47(\mathrm{t}, J=7.7 \mathrm{~Hz}, 1 \mathrm{H}), 7.35(\mathrm{t}, J=7.5 \mathrm{~Hz}, 1 \mathrm{H}), 7.17(\mathrm{t}, J=7.4 \mathrm{~Hz}, 1 \mathrm{H}), 4.50(\mathrm{~s}, 3 \mathrm{H})$, $2.96(\mathrm{~s}, 3 \mathrm{H}) .{ }^{13} \mathrm{C}$ NMR (101 MHz, DMSO- $\left.d_{6}\right) \delta 157.88,155.61,138.01,135.65$, $130.38,129.31,129.12,126.73,126.25,124.28,122.49,122.34,121.79,119.70$ 117.59, 39.74, 24.60. MS-ESI m/z: calcd for $\mathrm{C}_{17} \mathrm{H}_{14} \mathrm{~N}_{2}[\mathrm{M}+\mathrm{H}]^{+}$: 247.1952; found: 247.1977.

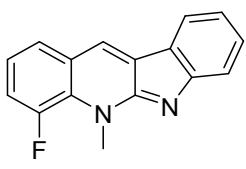

\section{4-fluoro-5-methyl-5H-indolo[2,3-b]quinoline (13)}

Yield, 57\%; red solid, m.p. 114.84-115.94 ${ }^{\circ} \mathrm{C} ;{ }^{1} \mathrm{H}$ NMR (400 MHz, DMSO- $\left.d_{6}\right) \delta 8.92$ (s, 1H), $8.12(\mathrm{~d}, J=7.5 \mathrm{~Hz}, 1 \mathrm{H}), 7.93(\mathrm{~d}, J=7.9 \mathrm{~Hz}, 1 \mathrm{H}), 7.65(\mathrm{~m}, 1 \mathrm{H}), 7.59$ (d, $J=$ $7.9 \mathrm{~Hz}, 1 \mathrm{H}), 7.55-7.47(\mathrm{~m}, 1 \mathrm{H}), 7.43$ (t, $J=7.9 \mathrm{~Hz}, 1 \mathrm{H}), 7.20$ (t, $J=7.3 \mathrm{~Hz}, 1 \mathrm{H})$, $4.48(\mathrm{~s}, 3 \mathrm{H}) .{ }^{13} \mathrm{C}$ NMR (101 MHz, DMSO- $\left.d_{6}\right) \delta 156.40,155.63,150.29,129.66$, $129.09(\mathrm{~d}, J=2.9 \mathrm{~Hz}), 128.06,126.77$ (d, $J=3.7 \mathrm{~Hz}), 126.30$ (d, $J=6.7 \mathrm{~Hz}), 124.13$, $123.59(\mathrm{~d}, J=2.7 \mathrm{~Hz}), 122.50(\mathrm{~d}, J=8.4 \mathrm{~Hz}), 122.07,120.11,117.95,117.76(\mathrm{~d}, J=$ $6.2 \mathrm{~Hz}), 37.21(\mathrm{~d}, J=15.4 \mathrm{~Hz})$. MS-ESI m/z: calcd for $\mathrm{C}_{16} \mathrm{H}_{11} \mathrm{FN}_{2}[\mathrm{M}+\mathrm{H}]^{+}:$251.1715; found: 251.1728 . 


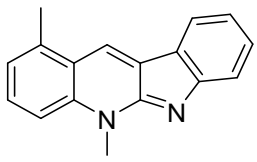

\section{1,5-dimethyl-5H-indolo[2,3-b]quinoline (14)}

Yield, 34\%; red solid, m.p. 167.53-169.43 ${ }^{\circ} \mathrm{C} ;{ }^{1} \mathrm{H}$ NMR (400 MHz, DMSO- $\left.d_{6}\right) \delta 9.05$ (s, 1H), $8.22(\mathrm{~d}, J=7.5 \mathrm{~Hz}, 1 \mathrm{H}), 7.77(\mathrm{~d}, J=8.7 \mathrm{~Hz}, 1 \mathrm{H}), 7.69$ (t, $J=8.7,1 \mathrm{H}), 7.59$ $(\mathrm{d}, J=7.9 \mathrm{~Hz}, 1 \mathrm{H}), 7.51-7.45(\mathrm{~m}, 1 \mathrm{H}), 7.32(\mathrm{~d}, J=7.1 \mathrm{~Hz}, 1 \mathrm{H}), 7.22-7.15(\mathrm{~m}, 1 \mathrm{H})$, $4.27(\mathrm{~s}, 3 \mathrm{H}), 2.79(\mathrm{~s}, 3 \mathrm{H}) .{ }^{13} \mathrm{C}$ NMR (101 MHz, DMSO-d $\left.d_{6}\right) \delta 155.77,155.47,137.49$, $137.35,130.62,129.04,126.79,125.90,124.59,123.55,122.01,119.48,119.42$, 117.50, 113.52, 33.40, 19.92. MS-ESI m/z: calcd for $\mathrm{C}_{17} \mathrm{H}_{14} \mathrm{~N}_{2}[\mathrm{M}+\mathrm{H}]^{+}$: 247.1912; found: 247.1921 .

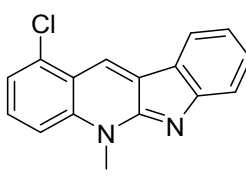

\section{1-chloro-5-methyl-5H-indolo[2,3-b]quinoline (15)}

Yield, 21\%; red solid, m.p. $162.05-165.25{ }^{\circ} \mathrm{C} ;{ }^{1} \mathrm{H}$ NMR (400 MHz, DMSO- $\left.d_{6}\right) \delta 9.08$ (s, 1H), $8.26(\mathrm{~d}, J=7.6 \mathrm{~Hz}, 1 \mathrm{H}), 7.91(\mathrm{~d}, J=8.8 \mathrm{~Hz}, 1 \mathrm{H}), 7.77(\mathrm{~d}, J=8.2 \mathrm{~Hz}, 1 \mathrm{H})$, $7.63-7.45(\mathrm{~m}, 3 \mathrm{H}), 7.18(\mathrm{~d}, J=6.7 \mathrm{~Hz}, 1 \mathrm{H}), 4.28(\mathrm{~s}, 3 \mathrm{H}) \cdot{ }^{13} \mathrm{C}$ NMR $(101 \mathrm{MHz}$, DMSO- $\left.d_{6}\right) \delta 155.96,138.18,132.87,130.98,129.81,128.50,124.31,122.80,122.53$ $120.05,117.96,117.74,114.99,40.20,39.78,33.74$. MS-ESI $\mathrm{m} / \mathrm{z}$ : calcd for $\mathrm{C}_{17} \mathrm{H}_{14} \mathrm{~N}_{2}[\mathrm{M}+\mathrm{H}]^{+}:$267.1336; found: 267.1348 .

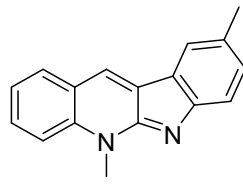

\section{5,9-dimethyl-5H-indolo[2,3-b]quinoline (16)}

Yield, 62\%; red solid, m.p. $149.86-152.56{ }^{\circ} \mathrm{C} ;{ }^{1} \mathrm{H}$ NMR (400 MHz, DMSO- $\left.d_{6}\right) \delta 8.86$ (s, 1H), $8.11(\mathrm{~d}, J=7.9 \mathrm{~Hz}, 1 \mathrm{H}), 7.97-7.90(\mathrm{~m}, 2 \mathrm{H}), 7.86-7.79(\mathrm{~m}, 1 \mathrm{H}), 7.49(\mathrm{~d}, J$ $=3.6 \mathrm{~Hz}, 1 \mathrm{H}), 7.47(\mathrm{~d}, J=4.2 \mathrm{~Hz}, 1 \mathrm{H}), 7.30(\mathrm{~d}, J=8.1 \mathrm{~Hz}, 1 \mathrm{H}), 4.27(\mathrm{~s}, 3 \mathrm{H}), 2.46(\mathrm{~s}$, 
$3 \mathrm{H}) .{ }^{13} \mathrm{C}$ NMR $\left(101 \mathrm{MHz}, \mathrm{DMSO}-d_{6}\right) \delta 155.59,153.68,137.08,131.00,130.36$, $130.29,129.07,128.38,127.44,124.38,122.10,121.91,120.58,117.27,115.24$ 33.09, 21.56. MS-ESI m/z: calcd for $\mathrm{C}_{17} \mathrm{H}_{14} \mathrm{~N}_{2}[\mathrm{M}+\mathrm{H}]^{+}:$: 247.1853; found: 247.1869 .

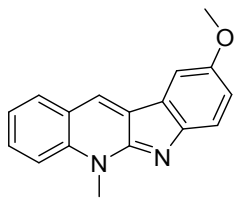

\section{9-methoxy-5-methyl-5H-indolo[2,3-b]quinoline (17)}

Yield, 51\%; red solid, m.p. 106.85-108.25 ${ }^{\circ} \mathrm{C} ;{ }^{1} \mathrm{H}$ NMR (400 MHz, DMSO- $\left.d_{6}\right) \delta 8.94$ (s, 1H), $8.11(\mathrm{~d}, J=6.5 \mathrm{~Hz}, 1 \mathrm{H}), 7.95(\mathrm{~d}, J=8.6 \mathrm{~Hz}, 1 \mathrm{H}), 7.86-7.82(\mathrm{~m}, 1 \mathrm{H}), 7.75$ $(\mathrm{d}, J=2.7 \mathrm{~Hz}, 1 \mathrm{H}), 7.51(\mathrm{~d}, J=1.8 \mathrm{~Hz}, 1 \mathrm{H}), 7.48(\mathrm{t}, J=3.8 \mathrm{~Hz}, 1 \mathrm{H}), 7.11(\mathrm{~d}, J=8.6$ $\mathrm{Hz}, 1 \mathrm{H}), 4.26$ (s, 3H), 3.85 (s, 3H). ${ }^{13} \mathrm{C}$ NMR (101 MHz, DMSO- $\left.d_{6}\right) \delta 154.56,154.08$, $148.61,137.08,131.32,130.47,130.11,127.29,124.36,122.32,120.52,117.65$ 117.17, 115.31, 105.95, 56.06, 33.34. MS-ESI m/z: calcd for $\mathrm{C}_{17} \mathrm{H}_{14} \mathrm{~N}_{2} \mathrm{O}[\mathrm{M}+\mathrm{H}]^{+}$: 263.1871; found: 263.1885 .

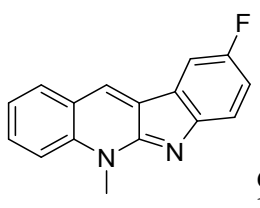

\section{9-fluoro-5-methyl-5H-indolo[2,3-b]quinoline (18)}

Yield, 53\%; red solid, m.p. 162.30-165.10 ${ }^{\circ} \mathrm{C} ;{ }^{1} \mathrm{H}$ NMR (400 MHz, DMSO- $\left.d_{6}\right) \delta 8.97$ (s, 1H), $8.12(\mathrm{~d}, J=8.0 \mathrm{~Hz}, 1 \mathrm{H}), 7.98(\mathrm{~d}, J=2.6 \mathrm{~Hz}, 1 \mathrm{H}), 7.96(\mathrm{~d}, J=2.5 \mathrm{~Hz}, 1 \mathrm{H})$, $7.86(\mathrm{~m}, 1 \mathrm{H}), 7.56(\mathrm{~d}, J=8.6 \mathrm{~Hz}, 1 \mathrm{H}), 7.50(\mathrm{t}, J=7.4 \mathrm{~Hz}, 1 \mathrm{H}), 7.31(\mathrm{~m}, 1 \mathrm{H}), 4.28(\mathrm{~s}$, 3H). ${ }^{13} \mathrm{C}$ NMR (101 MHz, DMSO- $\left.d_{6}\right) \delta 158.28,155.96,151.90,137.32,131.54$, 130.82, 130.59, $127.08(\mathrm{~d}, J=4.3 \mathrm{~Hz}), 124.71(\mathrm{~d}, J=10.0 \mathrm{~Hz}), 122.32,120.36$, $118.16(\mathrm{~d}, J=8.6 \mathrm{~Hz}), 116.17(\mathrm{~d}, J=24.1 \mathrm{~Hz}), 115.40,108.04(\mathrm{~d}, J=24.3 \mathrm{~Hz}), 33.19$. MS-ESI m/z: calcd for $\mathrm{C}_{16} \mathrm{H}_{11} \mathrm{FN}_{2}[\mathrm{M}+\mathrm{H}]^{+}$: 251.1698; found: 251.1704 . 


\section{9-chloro-5-methyl-5H-indolo[2,3-b]quinoline (19)}

Yield, 82\%; red solid, m.p. 226.87-228.47 ${ }^{\circ} \mathrm{C} ;{ }^{1} \mathrm{H}$ NMR (400 MHz, DMSO- $\left.d_{6}\right) \delta 9.08$ $(\mathrm{s}, 1 \mathrm{H}), 8.25(\mathrm{~s}, 1 \mathrm{H}), 8.16(\mathrm{~d}, J=7.9 \mathrm{~Hz}, 1 \mathrm{H}), 8.03(\mathrm{~d}, J=8.7 \mathrm{~Hz}, 1 \mathrm{H}), 7.90(\mathrm{t}, J=7.8$ $\mathrm{Hz}, 1 \mathrm{H}), 7.59$ (d, $J=8.4 \mathrm{~Hz}, 1 \mathrm{H}), 7.54(\mathrm{t}, J=7.5 \mathrm{~Hz}, 1 \mathrm{H}), 7.49(\mathrm{~d}, J=8.7 \mathrm{~Hz}, 1 \mathrm{H})$, $4.33(\mathrm{~s}, 3 \mathrm{H}) .{ }^{13} \mathrm{C}$ NMR $\left(101 \mathrm{MHz}, \mathrm{DMSO}-d_{6}\right) \delta 156.13,154.09,137.35,131.74$, $131.21,130.67,128.75,126.38,125.67,123.62,122.63,121.61,120.66,118.85$, 115.65, 33.38. MS-ESI m/z: calcd for $\mathrm{C}_{16} \mathrm{H}_{11} \mathrm{ClN}_{2}[\mathrm{M}+\mathrm{H}]^{+}$: 267.1135; found: 267.1142 .

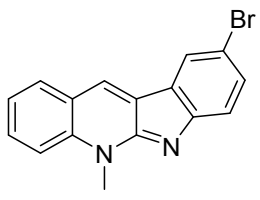

\section{9-bromo-5-methyl-5H-indolo[2,3-b]quinoline (20)}

Yield, 90\%; orange solid, m.p. 228.21-229.31 ${ }^{\circ} \mathrm{C} ;{ }^{1} \mathrm{H}$ NMR (400 MHz, DMSO- $\left.d_{6}\right) \delta$ $9.07(\mathrm{~s}, 1 \mathrm{H}), 8.37(\mathrm{~s}, 1 \mathrm{H}), 8.19-8.09(\mathrm{~m}, 1 \mathrm{H}), 8.02(\mathrm{~d}, J=8.4 \mathrm{~Hz}, 1 \mathrm{H}), 7.90(\mathrm{~s}, 1 \mathrm{H})$, $7.60(\mathrm{t}, J=8.3 \mathrm{~Hz}, 1 \mathrm{H}), 7.53(\mathrm{~d}, J=8.6 \mathrm{~Hz}, 2 \mathrm{H}), 4.32(\mathrm{~s}, 3 \mathrm{H}) .{ }^{13} \mathrm{C} \mathrm{NMR}(101 \mathrm{MHz}$, DMSO- $\left.d_{6}\right) \delta 155.99,154.37,137.34,131.74,131.40,131.25,130.67,126.32,126.23$, $124.50,122.65,120.69,119.38,115.66,111.33,33.39$. MS-ESI $\mathrm{m} / \mathrm{z}$ : calcd for $\mathrm{C}_{16} \mathrm{H}_{11} \mathrm{BrN}_{2}[\mathrm{M}+\mathrm{H}]^{+}: 311.0634$; found: 311.0642 .

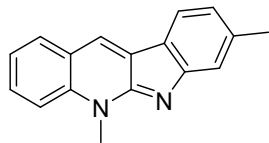

\section{5,8-dimethyl-5H-indolo[2,3-b]quinoline (21)}

Yield, 52\%; orange solid, m.p. $155.13-156.23{ }^{\circ} \mathrm{C} ;{ }^{1} \mathrm{H}$ NMR (400 MHz, DMSO- $\left.d_{6}\right) \delta$ $8.82(\mathrm{~s}, 1 \mathrm{H}), 8.10(\mathrm{~d}, J=8.0 \mathrm{~Hz}, 1 \mathrm{H}), 7.99(\mathrm{~d}, J=7.7 \mathrm{~Hz}, 1 \mathrm{H}), 7.94(\mathrm{~d}, J=8.6 \mathrm{~Hz}$, 1H), $7.81(\mathrm{~m}, 1 \mathrm{H}), 7.51-7.45(\mathrm{~m}, 1 \mathrm{H}), 7.39(\mathrm{~s}, 1 \mathrm{H}), 7.00(\mathrm{~d}, J=7.6 \mathrm{~Hz}, 1 \mathrm{H}), 4.28(\mathrm{~s}$,

3H), $2.48(\mathrm{~s}, 3 \mathrm{H}) .{ }^{13} \mathrm{C}$ NMR (101 MHz, DMSO- $\left.d_{6}\right) \delta 156.18,156.13,138.98,136.83$, 
$130.76,130.20,128.31,127.44,122.20,121.82,121.50,120.92,120.75,118.03$, 115.28, 33.14, 22.54. MS-ESI m/z: calcd for $\mathrm{C}_{17} \mathrm{H}_{14} \mathrm{~N}_{2}[\mathrm{M}+\mathrm{H}]^{+}$: 247.1670; found: 247.1676.

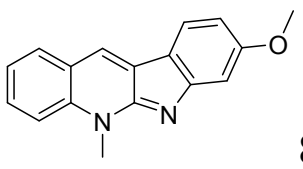

\section{8-methoxy-5-methyl-5H-indolo[2,3-b]quinoline (22)}

Yield, 63\%; brownish yellow solid, m.p. $148.29-148.69{ }^{\circ} \mathrm{C}$; ${ }^{1} \mathrm{H}$ NMR $(400 \mathrm{MHz}$, DMSO- $\left.d_{6}\right) \delta 8.72(\mathrm{~s}, 1 \mathrm{H}), 8.08(\mathrm{~d}, J=8.0 \mathrm{~Hz}, 1 \mathrm{H}), 8.00(\mathrm{~d}, J=8.4 \mathrm{~Hz}, 1 \mathrm{H}), 7.94(\mathrm{~d}, J$ $=8.6 \mathrm{~Hz}, 1 \mathrm{H}), 7.79(\mathrm{~m}, 1 \mathrm{H}), 7.47(\mathrm{t}, J=7.4 \mathrm{~Hz}, 1 \mathrm{H}), 7.15(\mathrm{~d}, J=2.3 \mathrm{~Hz}, 1 \mathrm{H}), 6.77(\mathrm{~d}$, $J=8.3 \mathrm{~Hz}, 1 \mathrm{H}), 4.28(\mathrm{~s}, 3 \mathrm{H}), 3.87(\mathrm{~s}, 3 \mathrm{H}) .{ }^{13} \mathrm{C}$ NMR $\left(101 \mathrm{MHz}, \mathrm{DMSO}-d_{6}\right) \delta 161.46$, $157.72,156.71,136.28,130.38,129.96,127.31,126.93,122.55,122.29,120.98$, 117.44, 115.31, 107.63, 102.07, 55.73, 33.13. MS-ESI $\mathrm{m} / \mathrm{z}$ : calcd for $\mathrm{C}_{17} \mathrm{H}_{14} \mathrm{~N}_{2} \mathrm{O}[\mathrm{M}+\mathrm{H}]^{+}:$263.1731; found: 263.1743 .

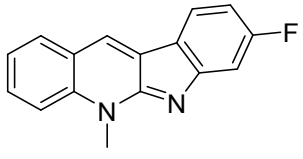

\section{8-fluoro-5-methyl-5H-indolo[2,3-b]quinoline (23)}

Yield, 50\%; yellow solid, m.p. $171.07-172.17{ }^{\circ} \mathrm{C} ;{ }^{1} \mathrm{H}$ NMR (400 MHz, DMSO- $\left.d_{6}\right) \delta$ $8.91(\mathrm{~s}, 1 \mathrm{H}), 8.14(\mathrm{~d}, J=6.0 \mathrm{~Hz}, 1 \mathrm{H}), 8.11(\mathrm{~d}, J=2.8 \mathrm{~Hz}, 1 \mathrm{H}), 7.98(\mathrm{~d}, J=8.7 \mathrm{~Hz}$, 1H), $7.84(\mathrm{t}, J=8.6 \mathrm{~Hz}, 1 \mathrm{H}), 7.54-7.48(\mathrm{~m}, 1 \mathrm{H}), 7.33(\mathrm{~d}, J=10.6 \mathrm{~Hz}, 1 \mathrm{H}), 6.98(\mathrm{~d}$, $J=10.4 \mathrm{~Hz}, 1 \mathrm{H}), 4.30(\mathrm{~s}, 3 \mathrm{H}) .{ }^{13} \mathrm{C}$ NMR $\left(101 \mathrm{MHz}, \mathrm{DMSO}-d_{6}\right) \delta 164.91,162.50$, $156.94,136.64,131.14,130.33,129.26(\mathrm{~d}, J=2.2 \mathrm{~Hz}), 126.44,122.99$ (d, $J=10.9$ $\mathrm{Hz}), 122.60,120.88,120.81,115.54,106.97$ (d, $J=24.3 \mathrm{~Hz}), 103.99$ (d, $J=23.5 \mathrm{~Hz})$, 33.31. MS-ESI m/z: calcd for $\mathrm{C}_{16} \mathrm{H}_{11} \mathrm{FN}_{2}[\mathrm{M}+\mathrm{H}]^{+}:$251.1680; found: 251.1688 .

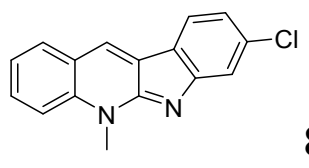

8-chloro-5-methyl-5H-indolo[2,3-b]quinoline (24) 
Yield, 51\%; yellow solid, m.p. 189.34-189.84 ${ }^{\circ} \mathrm{C} ;{ }^{1} \mathrm{H}$ NMR (400 MHz, DMSO- $\left.d_{6}\right) \delta$ $9.00(\mathrm{~s}, 1 \mathrm{H}), 8.15(\mathrm{~m}, 2 \mathrm{H}), 8.01(\mathrm{~d}, J=8.7 \mathrm{~Hz}, 1 \mathrm{H}), 7.88(\mathrm{t}, J=8.0 \mathrm{~Hz}, 1 \mathrm{H}), 7.59(\mathrm{~s}$, 1H), $7.53(\mathrm{t}, J=7.7 \mathrm{~Hz}, 1 \mathrm{H}), 7.19(\mathrm{~d}, J=8.1 \mathrm{~Hz}, 1 \mathrm{H}), 4.32(\mathrm{~s}, 3 \mathrm{H}) .{ }^{13} \mathrm{C}$ NMR $(101$ MHz, DMSO- $\left.d_{6}\right) \delta 156.66,137.03,133.46,131.48,130.52,130.37,126.27,123.07$, 123.02, 122.71, 120.88, 119.48, 117.21, 115.64, 33.40. MS-ESI m/z: calcd for $\mathrm{C}_{16} \mathrm{H}_{11} \mathrm{ClN}_{2}[\mathrm{M}+\mathrm{H}]^{+}:$267.1418; found: 267.1422 .

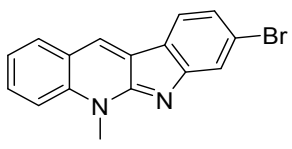

\section{8-bromo-5-methyl-5H-indolo[2,3-b]quinoline (25)}

Yield, 60\%; orange solid, m.p. 190.08-191.88 ${ }^{\circ} \mathrm{C} ;{ }^{1} \mathrm{H}$ NMR (400 MHz, DMSO- $\left.d_{6}\right) \delta$ $9.03(\mathrm{~s}, 1 \mathrm{H}), 8.16(\mathrm{~d}, J=6.6 \mathrm{~Hz}, 1 \mathrm{H}), 8.09(\mathrm{~d}, J=8.0 \mathrm{~Hz}, 1 \mathrm{H}), 8.02(\mathrm{~d}, J=8.7 \mathrm{~Hz}$, 1H), $7.89(\mathrm{t}, J=8.9 \mathrm{~Hz}, 1 \mathrm{H}), 7.74(\mathrm{~s}, 1 \mathrm{H}), 7.54(\mathrm{t}, J=7.4 \mathrm{~Hz}, 1 \mathrm{H}), 7.33(\mathrm{~d}, J=6.5 \mathrm{~Hz}$, 1H), $4.32(\mathrm{~s}, 3 \mathrm{H}) .{ }^{13} \mathrm{C}$ NMR (101 MHz, DMSO- $\left.d_{6}\right) \delta 156.87,156.47,137.12,131.53$, 130.55(2C), 126.31, 123.36(2C), 122.74, 122.20, 122.14, 120.90, 120.15, 115.67, 33.43. MS-ESI m/z: calcd for $\mathrm{C}_{16} \mathrm{H}_{11} \mathrm{BrN}_{2}[\mathrm{M}+\mathrm{H}]^{+}: 311.0961$; found: 311.0966 .

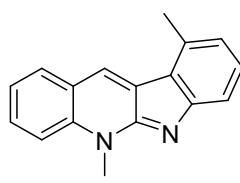

\section{5,10-dimethyl-5H-indolo[2,3-b]quinoline (26)}

Yield, 38\%; red solid, m.p. $175.49-178.19{ }^{\circ} \mathrm{C} ;{ }^{1} \mathrm{H}$ NMR (400 MHz, DMSO- $\left.d_{6}\right) \delta 8.83$ (s, 1H), $8.21(\mathrm{~d}, J=7.9 \mathrm{~Hz}, 1 \mathrm{H}), 7.95(\mathrm{~d}, J=8.6 \mathrm{~Hz}, 1 \mathrm{H}), 7.83(\mathrm{~m}, 1 \mathrm{H}), 7.49(\mathrm{t}, J=$ $7.5 \mathrm{~Hz}, 1 \mathrm{H}), 7.44$ (d, $J=7.9 \mathrm{~Hz}, 1 \mathrm{H}), 7.39$ (t, $J=7.6 \mathrm{~Hz}, 1 \mathrm{H}), 6.98$ (d, $J=7.1 \mathrm{~Hz}$, 1H), $4.29(\mathrm{~s}, 3 \mathrm{H}), 2.79(\mathrm{~s}, 3 \mathrm{H}) .{ }^{13} \mathrm{C}$ NMR $\left(101 \mathrm{MHz}, \mathrm{DMSO}-d_{6}\right) \delta 155.69,155.62$, $136.61,134.20,130.98,130.94,130.61,128.92,127.64,122.71,122.11,121.38$, 120.90, 115.35, 115.15, 33.13, 20.36. MS-ESI m/z: calcd for $\mathrm{C}_{17} \mathrm{H}_{14} \mathrm{~N}_{2}[\mathrm{M}+\mathrm{H}]^{+}$: 247.1943; found: 247.1950 . 


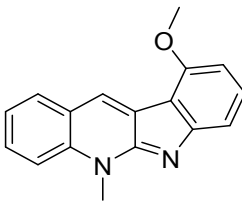

10-methoxy-5-methyl-5H-indolo[2,3-b]quinoline (27)

Yield, 53\%; orange solid, m.p. $188.95-190.75{ }^{\circ} \mathrm{C} ;{ }^{1} \mathrm{H}$ NMR (400 MHz, DMSO- $\left.d_{6}\right) \delta$ $8.74(\mathrm{~s}, 1 \mathrm{H}), 8.19(\mathrm{~d}, J=8.0 \mathrm{~Hz}, 1 \mathrm{H}), 7.95(\mathrm{~d}, J=8.6 \mathrm{~Hz}, 1 \mathrm{H}), 7.81(\mathrm{~m}, 1 \mathrm{H}), 7.51-$ $7.46(\mathrm{~m}, 1 \mathrm{H}), 7.46-7.41(\mathrm{~m}, 1 \mathrm{H}), 7.22(\mathrm{~d}, J=7.8 \mathrm{~Hz}, 1 \mathrm{H}), 6.79(\mathrm{~d}, J=8.1 \mathrm{~Hz}, 1 \mathrm{H})$, $4.30(\mathrm{~s}, 3 \mathrm{H}), 4.06(\mathrm{~s}, 3 \mathrm{H}) .{ }^{13} \mathrm{C}$ NMR $\left(101 \mathrm{MHz}, \mathrm{DMSO}-d_{6}\right) \delta 155.69,155.62,136.61$, $134.20,130.98,130.94,130.61,128.92,127.64,122.71,122.11,121.38,120.90$, 115.35, 115.15, 33.13, 20.36. MS-ESI m/z: calcd for $\mathrm{C}_{17} \mathrm{H}_{14} \mathrm{~N}_{2} \mathrm{O}[\mathrm{M}+\mathrm{H}]^{+}:$: 263.1905; found: 263.1911 .

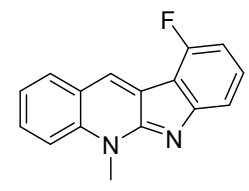

10-fluoro-5-methyl-5H-indolo[2,3-b]quinoline (28)

Yield, 60\%; orange solid, m.p. $189.40-190.10{ }^{\circ} \mathrm{C} ;{ }^{1} \mathrm{H}$ NMR (400 MHz, DMSO- $\left.d_{6}\right) \delta$ $8.89(\mathrm{~s}, 1 \mathrm{H}), 8.25(\mathrm{~d}, J=6.7 \mathrm{~Hz}, 1 \mathrm{H}), 8.00(\mathrm{~d}, J=8.7 \mathrm{~Hz}, 1 \mathrm{H}), 7.91-7.82(\mathrm{~m}, 1 \mathrm{H})$, $7.53(\mathrm{~d}, J=7.3 \mathrm{~Hz}, 1 \mathrm{H}), 7.51-7.46(\mathrm{~m}, 1 \mathrm{H}), 7.43(\mathrm{~d}, J=7.8 \mathrm{~Hz}, 1 \mathrm{H}), 7.00-6.93(\mathrm{~m}$, 1H), $4.32(\mathrm{~s}, 3 \mathrm{H}) .{ }^{13} \mathrm{C}$ NMR (101 MHz, DMSO- $\left.d_{6}\right) \delta 159.62,157.79(\mathrm{~d}, J=5.5 \mathrm{~Hz})$, 155.70, 136.91, $131.84(\mathrm{~d}, J=4.0 \mathrm{~Hz}), 131.60,130.67,130.23(\mathrm{~d}, J=8.6 \mathrm{~Hz}), 123.93$, 122.59, 121.00, 115.53, $114.05(\mathrm{~d}, J=3.0 \mathrm{~Hz}), 111.36(\mathrm{~d}, J=15.1 \mathrm{~Hz}), 105.75(\mathrm{~d}, J=$ 19.0 Hz), 33.50. MS-ESI $\mathrm{m} / \mathrm{z}$ : calcd for $\mathrm{C}_{16} \mathrm{H}_{11} \mathrm{FN}_{2}[\mathrm{M}+\mathrm{H}]^{+}$: 251.1700; found: 251.1706 .

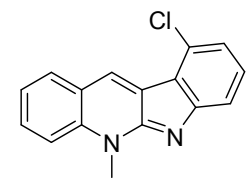

10-chloro-5-methyl-5H-indolo[2,3-b]quinoline (29) 
Yield, 55\%; orange solid, m.p. 186.19-187.79 ${ }^{\circ} \mathrm{C} ;{ }^{1} \mathrm{H}$ NMR (400 MHz, DMSO- $\left.d_{6}\right) \delta$ $9.11(\mathrm{~s}, 1 \mathrm{H}), 8.27(\mathrm{~d}, J=8.0 \mathrm{~Hz}, 1 \mathrm{H}), 7.99(\mathrm{~d}, J=8.7 \mathrm{~Hz}, 1 \mathrm{H}), 7.88(\mathrm{~m}, 1 \mathrm{H}), 7.56(\mathrm{t}$, $J=7.2 \mathrm{~Hz}, 1 \mathrm{H}), 7.54-7.50(\mathrm{~m}, 1 \mathrm{H}), 7.47(\mathrm{~d}, J=7.9 \mathrm{~Hz}, 1 \mathrm{H}), 7.20(\mathrm{~d}, J=7.7 \mathrm{~Hz}$, 1H), $4.32(\mathrm{~s}, 3 \mathrm{H}) .{ }^{13} \mathrm{C}$ NMR (101 MHz, DMSO- $\left.d_{6}\right) \delta 156.80,155.68,136.98,132.30$, $131.80,130.97,129.84,128.20,125.55,122.61,121.21,120.79,119.89,116.45$, 115.46, 33.44. MS-ESI $\mathrm{m} / \mathrm{z}$ : calcd for $\mathrm{C}_{16} \mathrm{H}_{11} \mathrm{ClN}_{2}[\mathrm{M}+\mathrm{H}]^{+}$: 267.1423; found: 267.1429 .

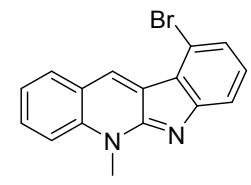

\section{0-bromo-5-methyl-5H-indolo[2,3-b]quinoline (30)}

Yield, 73\%; orange solid, m.p. 209.41-210.81 ${ }^{\circ} \mathrm{C}$; ${ }^{1} \mathrm{H}$ NMR (400 MHz, Chloroform- $d$ ) $\delta 8.98(\mathrm{~s}, 1 \mathrm{H}), 7.91(\mathrm{~d}, J=6.5 \mathrm{~Hz}, 1 \mathrm{H}), 7.67(\mathrm{~m}, 1 \mathrm{H}), 7.62(\mathrm{~d}, J=8.7 \mathrm{~Hz}, 1 \mathrm{H}), 7.59$ (d, $J=7.4 \mathrm{~Hz}, 1 \mathrm{H}), 7.35(\mathrm{t}, J=7.3 \mathrm{~Hz}, 1 \mathrm{H}), 7.29(\mathrm{t}, J=7.6 \mathrm{~Hz}, 1 \mathrm{H}), 7.25-7.16(\mathrm{~m}$, 1H), $4.25(\mathrm{~s}, 3 \mathrm{H}) .{ }^{13} \mathrm{C}$ NMR (101 MHz, Chloroform- $d$ ) $\delta$ 155.58, 154.89, 135.74, $130.50,129.87,129.54,128.52,126.27,122.43,121.95,121.08,119.63,116.07$, 115.50, 113.00, 32.05. MS-ESI m/z: calcd for $\mathrm{C}_{16} \mathrm{H}_{11} \mathrm{BrN}_{2}[\mathrm{M}+\mathrm{H}]^{+}$: 311.0991 ; found: 311.0995 .

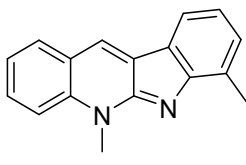

\section{5,7-dimethyl-5H-indolo[2,3-b]quinoline (31)}

Yield, 52\%; red solid, m.p. 188.26-189.76 ${ }^{\circ} \mathrm{C}$; ${ }^{1} \mathrm{H}$ NMR (400 MHz, DMSO- $\left.d_{6}\right) \delta 8.93$ (s, 1H), $8.15(\mathrm{~d}, J=7.9 \mathrm{~Hz}, 1 \mathrm{H}), 7.98(\mathrm{~d}, J=8.2 \mathrm{~Hz}, 2 \mathrm{H}), 7.85(\mathrm{~m}, 1 \mathrm{H}), 7.53-7.46$ (m, 1H), $7.32(\mathrm{~d}, J=7.3 \mathrm{~Hz}, 1 \mathrm{H}), 7.09$ (t, $J=7.4 \mathrm{~Hz}, 1 \mathrm{H}), 4.35(\mathrm{~s}, 3 \mathrm{H}), 2.60(\mathrm{~s}, 3 \mathrm{H})$.

${ }^{13} \mathrm{C}$ NMR $\left(101 \mathrm{MHz}, \mathrm{DMSO}-d_{6}\right) \delta 155.20,154.51,137.10,131.03,130.34,129.81$, 
129.31, 127.94, 126.45, 123.67, 122.20, 120.68, 119.62, 119.32, 115.34, 33.21, 17.56.

MS-ESI m/z: calcd for $\mathrm{C}_{17} \mathrm{H}_{14} \mathrm{~N}_{2}[\mathrm{M}+\mathrm{H}]^{+}:$247.1931; found: 247.1935 .

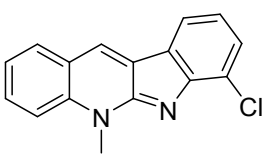

7-chloro-5-methyl-5H-indolo[2,3-b]quinoline (32)

Yield, 48\%; red solid, m.p. 241.48-242.88 ${ }^{\circ} \mathrm{C} ;{ }^{1} \mathrm{H}$ NMR (400 MHz, DMSO- $\left.d_{6}\right) \delta 9.12$ (s, 1H), $8.21(\mathrm{~d}, J=8.0,1 \mathrm{H}), 8.14(\mathrm{~d}, J=7.6 \mathrm{~Hz}, 1 \mathrm{H}), 8.07(\mathrm{~d}, J=8.6 \mathrm{~Hz}, 1 \mathrm{H}), 7.92$ (m, 1H), $7.60-7.52(\mathrm{~m}, 2 \mathrm{H}), 7.18(\mathrm{t}, J=7.7 \mathrm{~Hz}, 1 \mathrm{H}), 4.39(\mathrm{~s}, 3 \mathrm{H}) .{ }^{13} \mathrm{C}$ NMR $(101$ MHz, DMSO- $\left.d_{6}\right) \delta 155.89,151.76,137.29,131.77,131.36,130.67,128.72,127.08$, 126.10, 122.81, 121.48, 120.90, 120.65, 120.43, 115.77, 33.54. MS-ESI m/z: calcd for $\mathrm{C}_{16} \mathrm{H}_{11} \mathrm{ClN}_{2}[\mathrm{M}+\mathrm{H}]^{+}:$267.1429; found: 267.1435 .

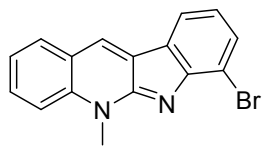

\section{7-bromo-5-methyl-5H-indolo[2,3-b]quinoline (33)}

Yield, 45\%; red solid, m.p. 245.56-247.36 ${ }^{\circ} \mathrm{C} ;{ }^{1} \mathrm{H}$ NMR (400 MHz, DMSO- $\left.d_{6}\right) \delta 9.12$ (s, 1H), $8.21(\mathrm{~d}, J=7.7 \mathrm{~Hz}, 1 \mathrm{H}), 8.19$ (d, $J=7.5 \mathrm{~Hz}, 1 \mathrm{H}), 8.08(\mathrm{~d}, J=8.6 \mathrm{~Hz}, 1 \mathrm{H})$, $7.96-7.90(\mathrm{~m}, 1 \mathrm{H}), 7.71(\mathrm{~d}, J=7.8 \mathrm{~Hz}, 1 \mathrm{H}), 7.57(\mathrm{t}, J=7.5 \mathrm{~Hz}, 1 \mathrm{H}), 7.13(\mathrm{~m}, 1 \mathrm{H})$, $4.40(\mathrm{~s}, 3 \mathrm{H}) .{ }^{13} \mathrm{C}$ NMR $\left(101 \mathrm{MHz}, \mathrm{DMSO}-d_{6}\right) \delta 155.78,153.16,137.28,131.78$, $131.68,131.45,130.67,127.31,125.78,122.83,121.19,120.89,120.87,115.79$, 110.83, 33.54. MS-ESI m/z: calcd for $\mathrm{C}_{16} \mathrm{H}_{11} \mathrm{BrN}_{2}[\mathrm{M}+\mathrm{H}]^{+}$: 313.0980; found: 313.0984 .

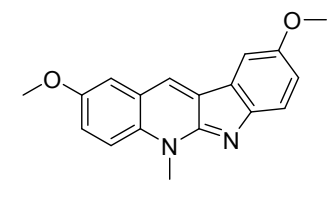

\section{2,9-dimethoxy-5-methyl-5H-indolo[2,3-b]quinoline (34)}

Yield, 52\%; red solid, m.p. $122.45-124.55{ }^{\circ} \mathrm{C} ;{ }^{1} \mathrm{H}$ NMR (400 MHz, DMSO- $\left.d_{6}\right) \delta 8.83$ (s, 1H), $7.87(\mathrm{~d}, J=9.3 \mathrm{~Hz}, 1 \mathrm{H}), 7.71(\mathrm{~s}, 1 \mathrm{H}), 7.58(\mathrm{~s}, 1 \mathrm{H}), 7.46(\mathrm{~d}, J=9.0 \mathrm{~Hz}, 2 \mathrm{H})$, 
$7.11(\mathrm{~d}, J=8.6 \mathrm{~Hz}, 1 \mathrm{H}), 4.24(\mathrm{~s}, 3 \mathrm{H}), 3.90(\mathrm{~s}, 3 \mathrm{H}), 3.85$ (s, 3H). ${ }^{13} \mathrm{C} \mathrm{NMR}(101 \mathrm{MHz}$, DMSO- $\left.d_{6}\right) \delta 154.92,154.29,153.65,150.23,132.13,128.88,128.12,124.30,121.07$, 120.63, 117.80, 117.14, 116.52, 111.02, 105.98, 56.13, 56.06, 33.13. MS-ESI m/z: calcd for $\mathrm{C}_{18} \mathrm{H}_{16} \mathrm{~N}_{2} \mathrm{O}_{2}[\mathrm{M}+\mathrm{H}]^{+}$: 293.2135; found: 293.2140 .

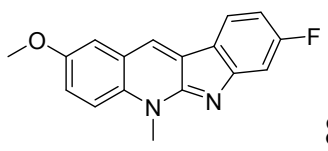

\section{8-fluoro-2-methoxy-5-methyl-5H-indolo[2,3-b]quinoline (35)}

Yield, 78\%; red solid, m.p. 193.20-193.50 ${ }^{\circ} \mathrm{C} ;{ }^{1} \mathrm{H}$ NMR (400 MHz, DMSO- $\left.d_{6}\right) \delta 8.87$ (s, 1H), $8.12(\mathrm{~m}, 1 \mathrm{H}), 7.95(\mathrm{~d}, J=9.4 \mathrm{~Hz}, 1 \mathrm{H}), 7.64(\mathrm{~s}, 1 \mathrm{H}), 7.50(\mathrm{~d}, J=9.4 \mathrm{~Hz}, 1 \mathrm{H})$, $7.30(\mathrm{~d}, J=10.7 \mathrm{~Hz}, 1 \mathrm{H}), 6.96(\mathrm{~m}, 1 \mathrm{H}), 4.30(\mathrm{~s}, 3 \mathrm{H}), 3.92(\mathrm{~s}, 3 \mathrm{H}) .{ }^{13} \mathrm{C}$ NMR $(101$ MHz, DMSO- $\left.d_{6}\right) \delta 162.57,157.52,156.57,154.73,131.60,128.58,126.82,122.97(\mathrm{~d}$, $J=11.2 \mathrm{~Hz}), 121.78,120.88,120.55,116.98,110.75,106.61(\mathrm{~d}, J=24.6 \mathrm{~Hz}), 103.72$ $(\mathrm{d}, J=23.4 \mathrm{~Hz}), 56.08,33.46 . \mathrm{MS}-\mathrm{ESI} \mathrm{m} / \mathrm{z}$ : calcd for $\mathrm{C}_{17} \mathrm{H}_{13} \mathrm{FN}_{2} \mathrm{O}[\mathrm{M}+\mathrm{H}]^{+}$: 281.1921; found: 281.1927.

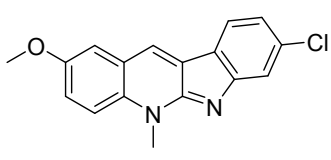

8-chloro-2-methoxy-5-methyl-5H-indolo[2,3-b]quinoline (36) Yield, 71\%; red solid, m.p. $210.08-210.48{ }^{\circ} \mathrm{C} ;{ }^{1} \mathrm{H}$ NMR (400 MHz, DMSO- $\left.d_{6}\right) \delta 8.95$ (s, 1H), $8.12(\mathrm{~d}, J=8.1 \mathrm{~Hz}, 1 \mathrm{H}), 7.97(\mathrm{~d}, J=9.4 \mathrm{~Hz}, 1 \mathrm{H}), 7.68-7.63(\mathrm{~m}, 1 \mathrm{H}), 7.55$ $(\mathrm{d}, J=7.5 \mathrm{~Hz}, 1 \mathrm{H}), 7.52-7.50(\mathrm{~m}, 1 \mathrm{H}), 7.16(\mathrm{~d}, J=8.3 \mathrm{~Hz}, 1 \mathrm{H}), 4.31(\mathrm{~s}, 3 \mathrm{H}), 3.92(\mathrm{~s}$, 3H). ${ }^{13} \mathrm{C}$ NMR (101 MHz, DMSO- $\left.d_{6}\right) \delta 156.86,156.25,154.75,133.47,132.00$, $129.66,126.61,123.00,122.77,121.77,121.30,119.07,117.09,116.99,110.75$, 56.09, 33.55. MS-ESI m/z: calcd for $\mathrm{C}_{17} \mathrm{H}_{13} \mathrm{ClN}_{2} \mathrm{O}[\mathrm{M}+\mathrm{H}]^{+}$: 297.1657; found: 297.1662 . 
8-bromo-2-methoxy-5-methyl-5H-indolo[2,3-b]quinoline (37) Yield, 81\%; orange solid, m.p. 207.05-208.65 ${ }^{\circ} \mathrm{C} ;{ }^{1} \mathrm{H}$ NMR (400 MHz, DMSO- $\left.d_{6}\right) \delta$ $8.97(\mathrm{~s}, 1 \mathrm{H}), 8.06(\mathrm{~d}, J=7.9 \mathrm{~Hz}, 1 \mathrm{H}), 7.97(\mathrm{~d}, J=9.3 \mathrm{~Hz}, 1 \mathrm{H}), 7.71(\mathrm{~s}, 1 \mathrm{H}), 7.66(\mathrm{~s}$, 1H), $7.53(\mathrm{~d}, J=9.2 \mathrm{~Hz}, 1 \mathrm{H}), 7.29(\mathrm{~d}, J=8.0 \mathrm{~Hz}, 1 \mathrm{H}), 4.31(\mathrm{~s}, 3 \mathrm{H}), 3.92(\mathrm{~s}, 3 \mathrm{H}) .{ }^{13} \mathrm{C}$ NMR $\left(101 \mathrm{MHz}, \mathrm{DMSO}-d_{6}\right) \delta 157.07,156.05,154.75,132.07,129.78,126.63$, $123.32,123.04,122.15,121.77,121.34,119.93,117.10,110.75,56.09,33.56$. MS-ESI m/z: calcd for $\mathrm{C}_{17} \mathrm{H}_{13} \mathrm{BrN}_{2} \mathrm{O}[\mathrm{M}+\mathrm{H}]^{+}:$341.1109; found: 341.1115 .

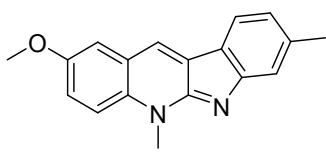

\section{2-methoxy-5,8-dimethyl-5H-indolo[2,3-b]quinoline (38)}

Yield, 62\%; red solid, m.p. 149.38-149.98 ${ }^{\circ} \mathrm{C} ;{ }^{1} \mathrm{H}$ NMR (400 MHz, DMSO- $\left.d_{6}\right) \delta 8.74$ (s, 1H), $7.96(\mathrm{~d}, J=7.7 \mathrm{~Hz}, 1 \mathrm{H}), 7.87(\mathrm{~d}, J=9.3 \mathrm{~Hz}, 1 \mathrm{H}), 7.59(\mathrm{~s}, 1 \mathrm{H}), 7.44(\mathrm{~d}, J=$ $9.3 \mathrm{~Hz}, 1 \mathrm{H}), 7.36(\mathrm{~s}, 1 \mathrm{H}), 6.97$ (d, J= $7.9 \mathrm{~Hz}, 1 \mathrm{H}), 4.25$ (s, 3H), 3.89 (s, 3H), 2.47 (s, $3 \mathrm{H}) .{ }^{13} \mathrm{C}$ NMR (101 MHz, DMSO- $\left.d_{6}\right) \delta 156.35,155.74,154.43,138.96,131.70$, $127.75,127.64,121.55,121.51,121.45,120.55,120.29,117.80,116.63,110.73$, 56.01, 33.25, 22.55. MS-ESI m/z: calcd for $\mathrm{C}_{18} \mathrm{H}_{16} \mathrm{~N}_{2} \mathrm{O}[\mathrm{M}+\mathrm{H}]^{+}$: 277.1911; found: 277.1915 .

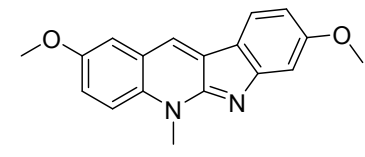

\section{2,8-dimethoxy-5-methyl-5H-indolo[2,3-b]quinoline (39)}

Yield, 47\%; orange solid, m.p. $143.71-146.71{ }^{\circ} \mathrm{C} ;{ }^{1} \mathrm{H}$ NMR (400 MHz, DMSO- $\left.d_{6}\right) \delta$ $8.66(\mathrm{~s}, 1 \mathrm{H}), 7.97(\mathrm{~d}, J=8.3 \mathrm{~Hz}, 1 \mathrm{H}), 7.88(\mathrm{~d}, J=9.3 \mathrm{~Hz}, 1 \mathrm{H}), 7.58(\mathrm{~s}, 1 \mathrm{H}), 7.43(\mathrm{dd}$, $J=9.3,2.9 \mathrm{~Hz}, 1 \mathrm{H}), 7.11(\mathrm{~s}, 1 \mathrm{H}), 6.74(\mathrm{~d}, J=8.4 \mathrm{~Hz}, 1 \mathrm{H}), 4.26(\mathrm{~s}, 3 \mathrm{H}), 3.90(\mathrm{~s}, 3 \mathrm{H})$, $3.86(\mathrm{~s}, 3 \mathrm{H}) .{ }^{13} \mathrm{C}$ NMR $\left(101 \mathrm{MHz}, \mathrm{DMSO}-d_{6}\right) \delta 161.47,157.86,156.29,154.52$, $131.13,127.61,126.31,122.52,121.82,119.91,117.14,116.69,110.53,107.36$, 
101.70, 56.00, 55.69, 33.25. MS-ESI m/z: calcd for $\mathrm{C}_{18} \mathrm{H}_{16} \mathrm{~N}_{2} \mathrm{O}_{2}[\mathrm{M}+\mathrm{H}]^{+}$: 293.1953; found: 293.1959 .

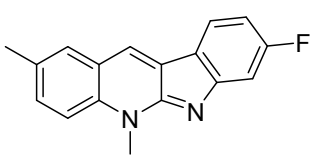

\section{8-fluoro-2,5-dimethyl-5H-indolo[2,3-b]quinoline (40)}

Yield, 50\%; orange solid, m.p. 156.91-148.51 ${ }^{\circ} \mathrm{C} ;{ }^{1} \mathrm{H}$ NMR (400 MHz, DMSO- $\left.d_{6}\right) \delta$ $8.79(\mathrm{~s}, 1 \mathrm{H}), 8.11(\mathrm{~m}, 1 \mathrm{H}), 7.86(\mathrm{~d}, J=8.5 \mathrm{~Hz}, 2 \mathrm{H}), 7.64(\mathrm{~d}, J=8.8 \mathrm{~Hz}, 1 \mathrm{H}), 7.31(\mathrm{~d}$, $J=10.6 \mathrm{~Hz}, 1 \mathrm{H}), 6.96(\mathrm{~m}, 1 \mathrm{H}), 4.26(\mathrm{~s}, 3 \mathrm{H}), 2.48(\mathrm{~s}, 3 \mathrm{H}) .{ }^{13} \mathrm{C}$ NMR $(101 \mathrm{MHz}$ DMSO- $\left.d_{6}\right) \delta 157.23(\mathrm{~d}, J=12.8 \mathrm{~Hz}), 156.78,134.83,132.57,131.75,129.50,128.89$, $128.87,126.36,122.93(\mathrm{~d}, J=10.9 \mathrm{~Hz}), 120.86,120.79,115.39,106.74(\mathrm{~d}, J=24.3$ $\mathrm{Hz}), 103.83(\mathrm{~d}, J=23.5 \mathrm{~Hz}), 33.26,20.78$. MS-ESI m/z: calcd for $\mathrm{C}_{17} \mathrm{H}_{13} \mathrm{FN}_{2}[\mathrm{M}+\mathrm{H}]^{+}$: 265.1760; found: 265.1766 .

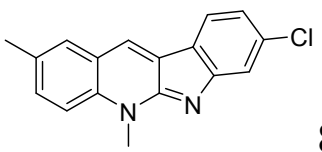

\section{8-chloro-2,5-dimethyl-5H-indolo[2,3-b]quinoline (41)}

Yield, 68\%; red solid, m.p. 183.63-184.53 ${ }^{\circ} \mathrm{C} ;{ }^{1} \mathrm{H}$ NMR (400 MHz, DMSO- $\left.d_{6}\right) \delta 8.90$ (s, 1H), $8.12(\mathrm{~d}, J=8.0 \mathrm{~Hz}, 1 \mathrm{H}), 7.90(\mathrm{~d}, J=6.3 \mathrm{~Hz}, 2 \mathrm{H}), 7.69(\mathrm{~d}, J=8.6 \mathrm{~Hz}, 1 \mathrm{H})$, $7.56(\mathrm{~s}, 1 \mathrm{H}), 7.17(\mathrm{~d}, J=8.0 \mathrm{~Hz}, 1 \mathrm{H}), 4.28(\mathrm{~s}, 3 \mathrm{H}), 3.34(\mathrm{~s}, 3 \mathrm{H}) .{ }^{13} \mathrm{C}$ NMR $(101 \mathrm{MHz}$, DMSO- $\left.d_{6}\right) \delta 156.70,156.52,135.27,133.35,132.94,131.88,129.98,129.68,126.22$ 123.04, 122.97, 120.89, 119.24, 117.09, 115.51, 33.36, 20.80. MS-ESI m/z: calcd for $\mathrm{C}_{17} \mathrm{H}_{13} \mathrm{ClN}_{2}[\mathrm{M}+\mathrm{H}]^{+}:$281.1531; found: 281.1537 .

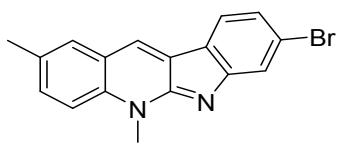

\section{8-bromo-2,5-dimethyl-5H-indolo[2,3-b]quinoline (42)}

Yield, 75\%; red solid, m.p. 173.44-174.63 ${ }^{\circ} \mathrm{C} ;{ }^{1} \mathrm{H}$ NMR (400 MHz, DMSO- $\left.d_{6}\right) \delta 8.96$ (s, 1H), 8.09 (d, $J=8.3 \mathrm{~Hz}, 1 \mathrm{H}), 7.94(\mathrm{~s}, 2 \mathrm{H}), 7.72(\mathrm{~s}, 2 \mathrm{H}), 7.31(\mathrm{~d}, J=8.1 \mathrm{~Hz}, 1 \mathrm{H})$, $4.41-4.16(\mathrm{~m}, 3 \mathrm{H}), 3.32(\mathrm{~s}, 3 \mathrm{H}) .{ }^{13} \mathrm{C}$ NMR $\left(101 \mathrm{MHz}, \mathrm{DMSO}-d_{6}\right) \delta$ 156.87, 156.33, 
$135.38,133.04,131.95,130.22,129.73,126.27,123.35,122.05,121.99,120.95$, 120.02, 115.59, 99.99, 33.42, 20.82. MS-ESI m/z: calcd for $\mathrm{C}_{17} \mathrm{H}_{13} \mathrm{BrN}_{2}[\mathrm{M}+\mathrm{H}]^{+}$: 327.1132; found: 327.1139 .

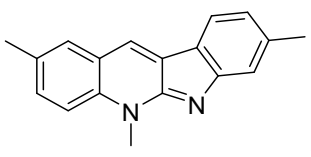

\section{2,5,8-trimethyl-5H-indolo[2,3-b]quinoline (43)}

Yield, 53\%; red solid, m.p. $184.27-186.37{ }^{\circ} \mathrm{C} ;{ }^{1} \mathrm{H}$ NMR (400 MHz, DMSO- $\left.d_{6}\right) \delta 8.72$ (s, 1H), $7.98(\mathrm{~d}, J=7.7 \mathrm{~Hz}, 1 \mathrm{H}), 7.86-7.80(\mathrm{~m}, 2 \mathrm{H}), 7.62(\mathrm{dd}, J=8.7 \mathrm{~Hz}, 1 \mathrm{H}), 7.37$ (s, 1H), $6.98(\mathrm{~d}, J=7.8 \mathrm{~Hz}, 1 \mathrm{H}), 4.25(\mathrm{~s}, 3 \mathrm{H}), 2.48(\mathrm{~d}, J=2.5 \mathrm{~Hz}, 6 \mathrm{H}) .{ }^{13} \mathrm{C}$ NMR (101 MHz, DMSO- $\left.d_{6}\right) \delta 156.22,156.02,138.84,135.06,132.17,131.29,129.45$, 127.96, 127.41, 121.80, 121.45, 120.76, 120.71, 117.92, 115.15, 33.10, 22.53, 20.79. MS-ESI m/z: calcd for $\mathrm{C}_{18} \mathrm{H}_{16} \mathrm{~N}_{2}[\mathrm{M}+\mathrm{H}]^{+}:$261.2120; found: 261.2122 .

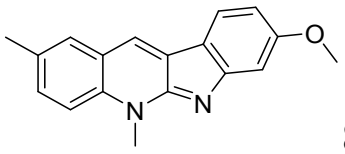

\section{8-methoxy-2,5-dimethyl-5H-indolo[2,3-b]quinoline (44)}

Yield, $42 \%$; red solid, m.p. $156.72-158.62{ }^{\circ} \mathrm{C} ;{ }^{1} \mathrm{H}$ NMR (400 MHz, DMSO- $\left.d_{6}\right) \delta 8.63$ (s, 1H), $7.99(\mathrm{~d}, J=8.3 \mathrm{~Hz}, 1 \mathrm{H}), 7.86-7.81(\mathrm{~m}, 2 \mathrm{H}), 7.61(\mathrm{~d}, J=8.8 \mathrm{~Hz}, 1 \mathrm{H}), 7.12$ (d, $J=2.4 \mathrm{~Hz}, 1 \mathrm{H}), 6.75(\mathrm{~d}, J=8.3 \mathrm{~Hz}, 1 \mathrm{H}), 4.25(\mathrm{~s}, 3 \mathrm{H}), 3.86(\mathrm{~s}, 3 \mathrm{H}), 2.48(\mathrm{~s}, 3 \mathrm{H})$.

${ }^{13} \mathrm{C}$ NMR (101 MHz, DMSO- $\left.d_{6}\right) \delta 161.39,157.70,156.57,134.49,131.81,131.40$, $129.23,127.26,126.63,122.51,120.99,117.42,115.20,107.45,101.91,55.70,33.11$, 20.81. MS-ESI m/z: calcd for $\mathrm{C}_{18} \mathrm{H}_{16} \mathrm{~N}_{2} \mathrm{O}[\mathrm{M}+\mathrm{H}]^{+}$: 277.2037; found: 277.2040 .

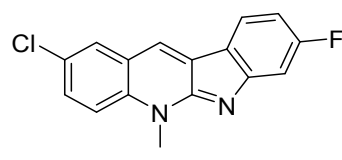

\section{2-chloro-8-fluoro-5-methyl-5H-indolo[2,3-b]quinoline (45)}

Yield, 70\%; red solid, m.p. $206.78-208.78{ }^{\circ} \mathrm{C} ;{ }^{1} \mathrm{H}$ NMR (400 MHz, DMSO- $\left.d_{6}\right) \delta 8.86$ (s, 1H), 8.21 (s, 1H), $8.11(\mathrm{~d}, J=8.4 \mathrm{~Hz}, 1 \mathrm{H}), 8.01(\mathrm{~d}, J=9.3 \mathrm{~Hz}, 1 \mathrm{H}), 7.95-7.79$ (m, 1H), $7.34(\mathrm{~m}, 1 \mathrm{H}), 7.09-6.92(\mathrm{~m}, 1 \mathrm{H}), 4.30(\mathrm{~d}, J=4.5 \mathrm{~Hz}, 3 \mathrm{H}) .{ }^{13} \mathrm{C}$ NMR $(101 \mathrm{MHz}$, 
DMSO- $\left.d_{6}\right) \delta 157.54,157.42,156.76,135.27,130.68,128.78,128.01,127.53,126.62$, $123.28(\mathrm{~d}, J=11.0 \mathrm{~Hz}), 121.93,120.60,117.70,107.34(\mathrm{~d}, J=24.2 \mathrm{~Hz}), 104.22(\mathrm{~d}, J$ $=23.5 \mathrm{~Hz}$ ), 33.55. MS-ESI m/z: calcd for $\mathrm{C}_{16} \mathrm{H}_{10} \mathrm{ClFN}_{2}[\mathrm{M}+\mathrm{H}]^{+}$: 285.1372; found: 285.1377.

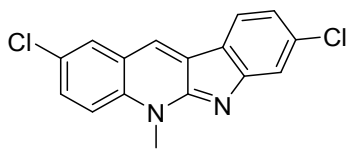

\section{2,8-dichloro-5-methyl-5H-indolo[2,3-b]quinoline (46)}

Yield, 68\%; red solid, m.p. $243.96-245.16{ }^{\circ} \mathrm{C}$; ${ }^{1} \mathrm{H}$ NMR (400 MHz, DMSO- $\left.d_{6}\right) \delta 8.97$ (s, 1H), $8.26(\mathrm{~s}, 1 \mathrm{H}), 8.14(\mathrm{~d}, J=8.1 \mathrm{~Hz}, 1 \mathrm{H}), 8.06(\mathrm{~d}, J=9.0 \mathrm{~Hz}, 1 \mathrm{H}), 7.90(\mathrm{~m}, 1 \mathrm{H})$, $7.61(\mathrm{~s}, 1 \mathrm{H}), 7.22(\mathrm{~d}, J=8.0 \mathrm{~Hz}, 1 \mathrm{H}), 4.32(\mathrm{~s}, 3 \mathrm{H}) .{ }^{13} \mathrm{C}$ NMR $(101 \mathrm{MHz}$, Chloroform- $d$ ) $\delta 170.14,155.51,134.17,129.59,127.63,126.63,125.96,120.80$, 119.32, 116.98, 114.66, 59.38, 52.41, 32.21, 20.03, 13.18. MS-ESI m/z: calcd for $\mathrm{C}_{16} \mathrm{H}_{10} \mathrm{Cl}_{2} \mathrm{~N}_{2}[\mathrm{M}+\mathrm{H}]^{+}$: 301.1112; found: 301.1115 .

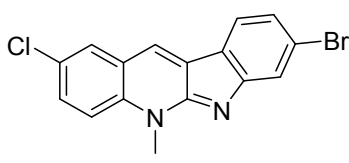

8-bromo-2-chloro-5-methyl-5H-indolo[2,3-b]quinoline (47) Yield, 72\%; red solid, m.p. $268.87-270.17{ }^{\circ} \mathrm{C} ;{ }^{1} \mathrm{H}$ NMR (400 MHz, DMSO- $\left.d_{6}\right) \delta 9.01$ (s, 1H), $8.28(\mathrm{~s}, 1 \mathrm{H}), 8.10(\mathrm{~d}, J=9.4 \mathrm{~Hz}, 2 \mathrm{H}), 7.91(\mathrm{~m}, 1 \mathrm{H}), 7.77(\mathrm{~s}, 1 \mathrm{H}), 7.36(\mathrm{~m}$, 1H), 4.33 (s, 3H). ${ }^{13} \mathrm{C}$ NMR (101 MHz, Chloroform- $d$ ) $\delta$ 170.85, 155.23, 154.82, 134.27, 130.04, 127.79, 126.94, 122.41, 121.27, 119.50, 115.06, 59.68, 52.51, 32.44, 19.98, 13.09. MS-ESI $\mathrm{m} / \mathrm{z}$ : calcd for $\mathrm{C}_{16} \mathrm{H}_{10} \mathrm{BrClN}_{2}[\mathrm{M}+\mathrm{H}]^{+}$: 347.0470; found: 347.0475 .

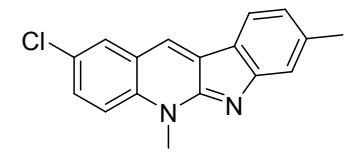

\section{2-chloro-5,8-dimethyl-5H-indolo[2,3-b]quinoline (48)}

Yield, 49\%; red solid, m.p. $200.04-201.64{ }^{\circ} \mathrm{C} ;{ }^{1} \mathrm{H}$ NMR (400 MHz, DMSO- $\left.d_{6}\right) \delta 8.73$ (s, 1H), 8.16 (s, 1H), 7.96 (d, $J=3.0 \mathrm{~Hz}, 1 \mathrm{H}), 7.94(\mathrm{~d}, J=4.3 \mathrm{~Hz}, 1 \mathrm{H}), 7.79$ (d, $J=$ 
$9.2 \mathrm{~Hz}, 1 \mathrm{H}), 7.37(\mathrm{~s}, 1 \mathrm{H}), 7.00(\mathrm{~d}, J=7.8 \mathrm{~Hz}, 1 \mathrm{H}), 4.26(\mathrm{~s}, 3 \mathrm{H}), 2.47(\mathrm{~s}, 3 \mathrm{H}) .{ }^{13} \mathrm{C}$ NMR $\left(101 \mathrm{MHz}, \mathrm{DMSO}-d_{6}\right) \delta 156.36,155.91,139.56,135.43,130.23,128.64$, $128.51,126.99,126.15,121.82,121.65,121.58,121.21,118.17,117.35,33.35,22.54$. MS-ESI m/z: calcd for $\mathrm{C}_{17} \mathrm{H}_{13} \mathrm{ClN}_{2}[\mathrm{M}+\mathrm{H}]^{+}:$281.1492; found: 281.1497 .

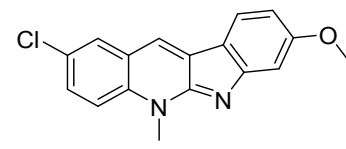

\section{2-chloro-8-methoxy-5-methyl-5H-indolo[2,3-b]quinoline (49)}

Yield, 62\%; red solid, m.p. 180.11-181.23 ${ }^{\circ} \mathrm{C} ;{ }^{1} \mathrm{H}$ NMR (400 MHz, DMSO- $\left.d_{6}\right) \delta 8.64$ $(\mathrm{s}, 1 \mathrm{H}), 8.14(\mathrm{~s}, 1 \mathrm{H}), 7.97(\mathrm{~d}, J=7.1 \mathrm{~Hz}, 1 \mathrm{H}), 7.94(\mathrm{~d}, J=8.0 \mathrm{~Hz}, 1 \mathrm{H}), 7.78(\mathrm{~d}, J=$ $9.1 \mathrm{~Hz}, 1 \mathrm{H}), 7.13(\mathrm{~s}, 1 \mathrm{H}), 6.77(\mathrm{~d}, J=8.4 \mathrm{~Hz}, 1 \mathrm{H}), 4.26(\mathrm{~s}, 3 \mathrm{H}), 3.87(\mathrm{~s}, 3 \mathrm{H}) .{ }^{13} \mathrm{C}$ NMR $\left(101 \mathrm{MHz}, \mathrm{DMSO}-d_{6}\right) \delta 161.77,157.96,156.50,134.85,129.84,128.39$, $128.35,126.27,125.57,122.78,122.06,117.39,117.16,107.93,102.17,55.74,33.34$. MS-ESI m/z: calcd for $\mathrm{C}_{17} \mathrm{H}_{13} \mathrm{ClN}_{2} \mathrm{O}[\mathrm{M}+\mathrm{H}]^{+}:$297.1528; found: 297.1531 . 
4. Spectrums of the representative compounds.

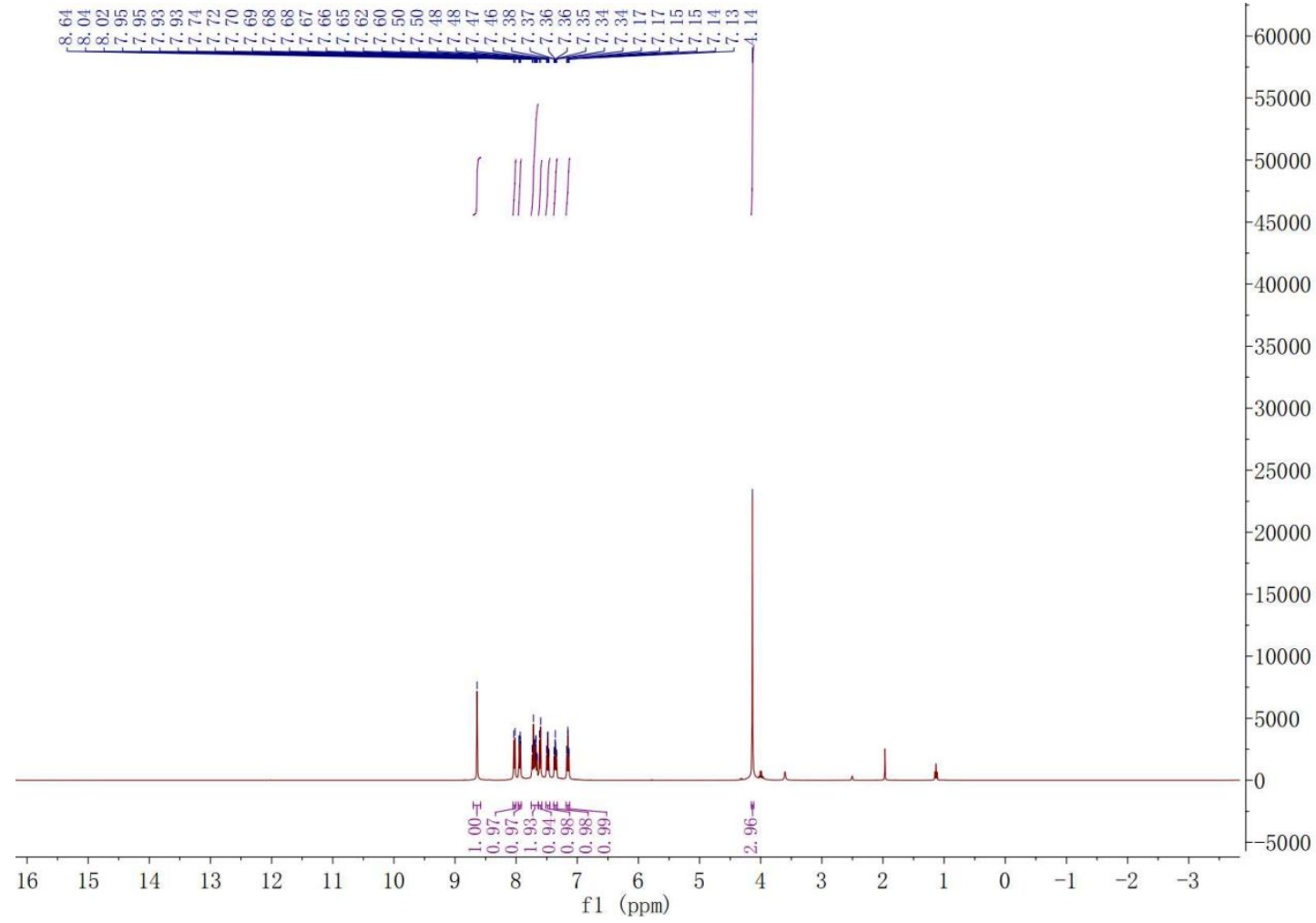

The ${ }^{1} \mathrm{H}$ NMR spectrogram of compound $\mathbf{1}$

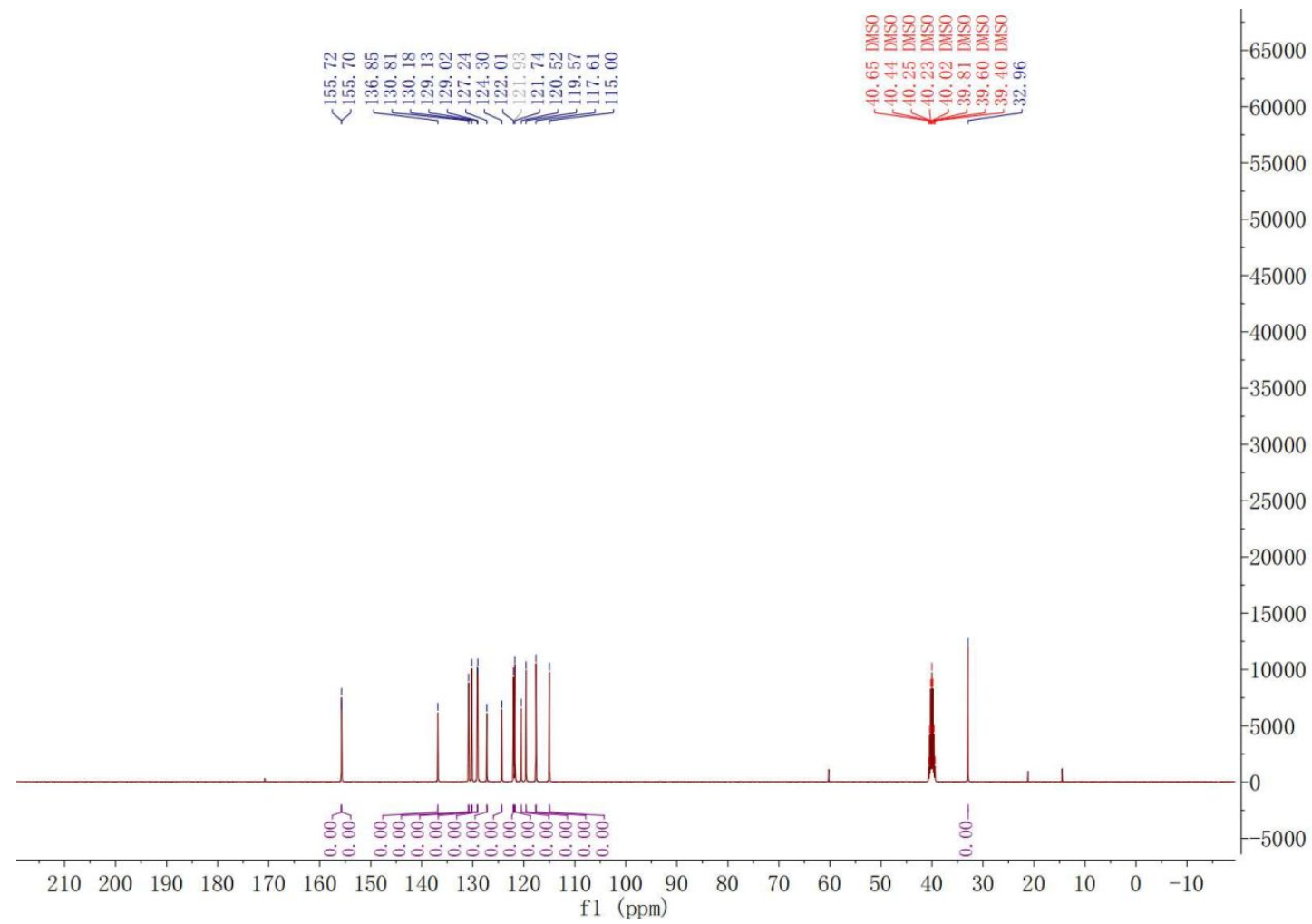

The ${ }^{13} \mathrm{C}$ NMR spectrogram of compound 1 


\section{Generic Display Report}

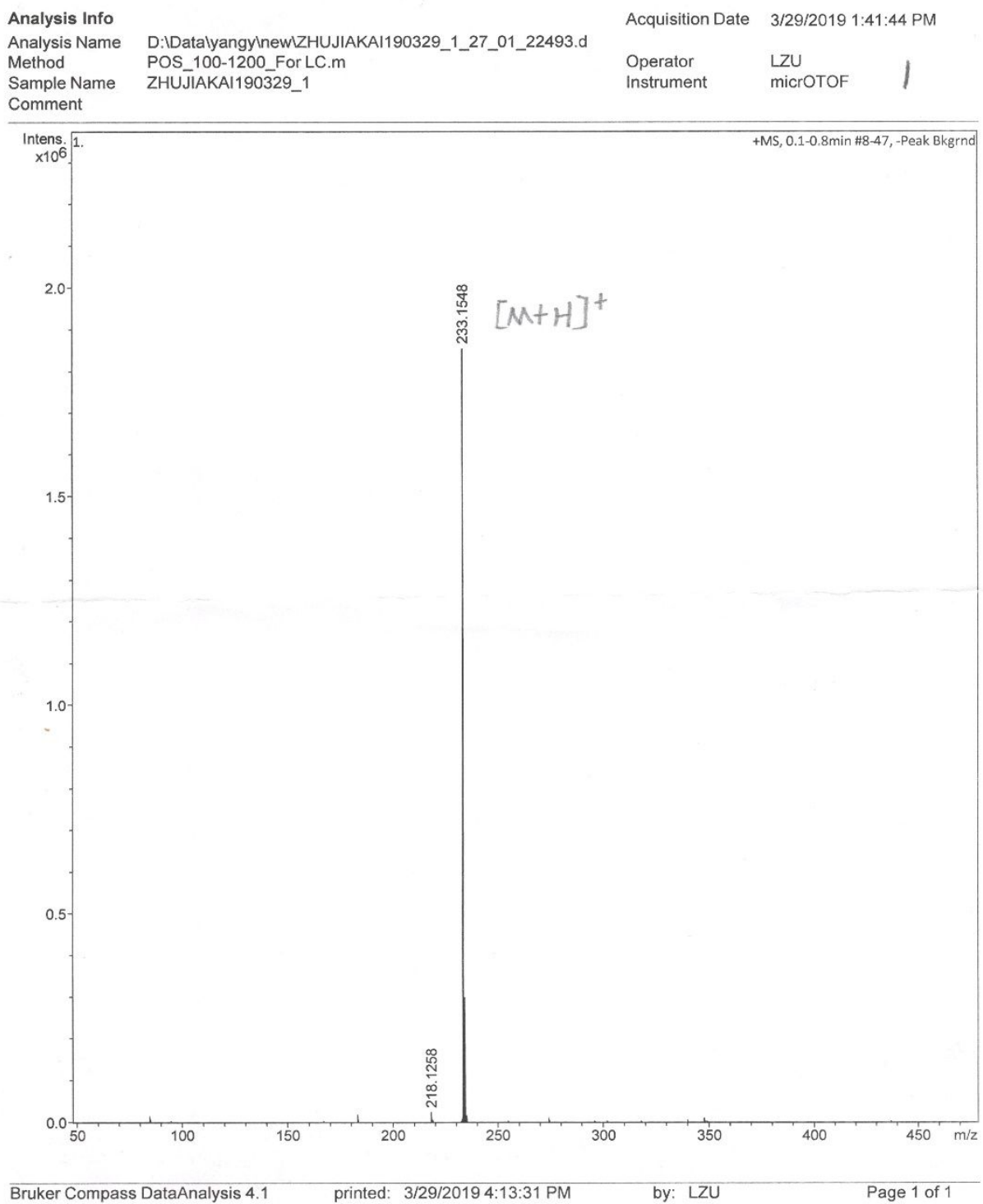

The Mass spectrogram of compound 1 


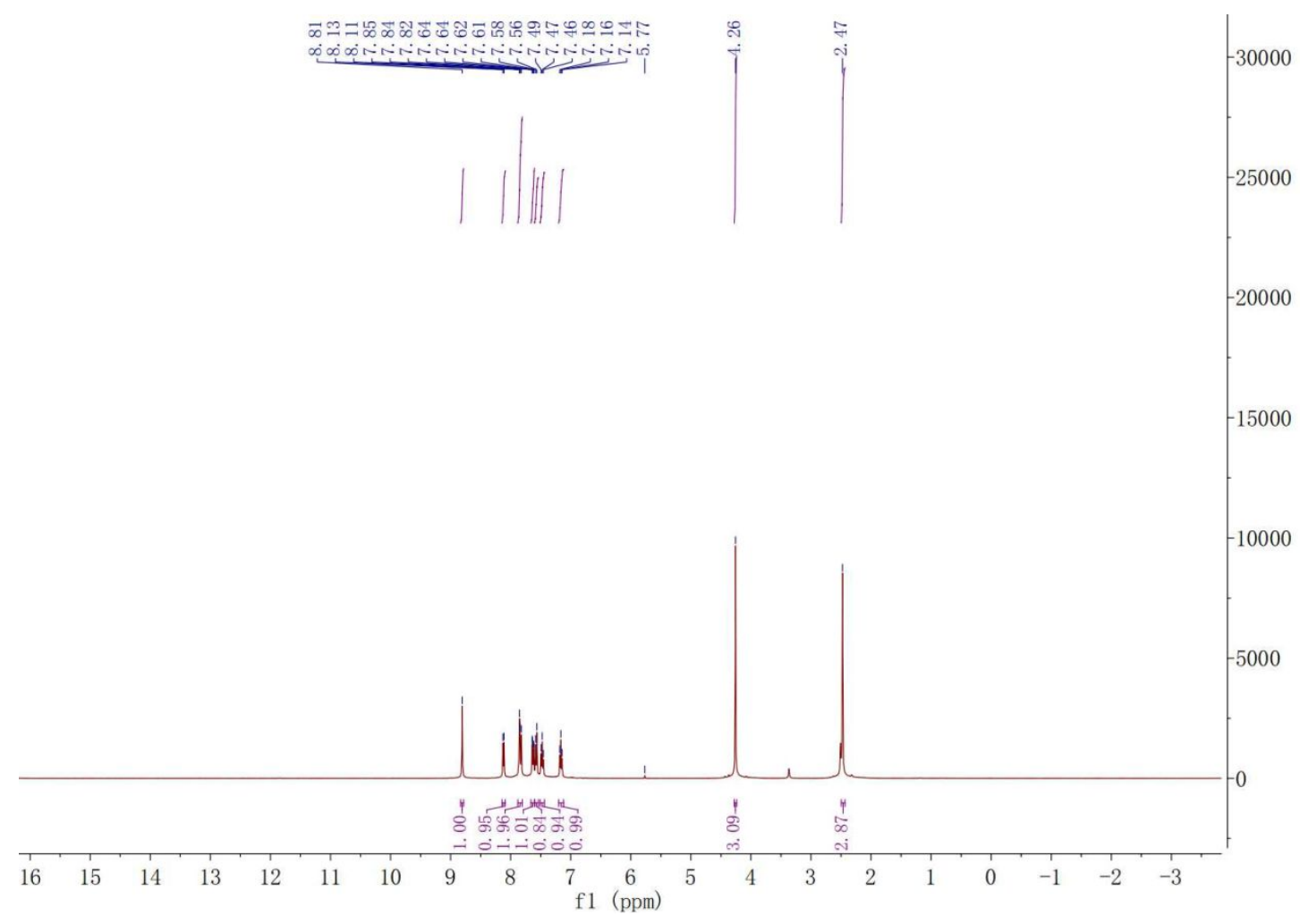

The ${ }^{1} \mathrm{H}$ NMR spectrogram of compound 2

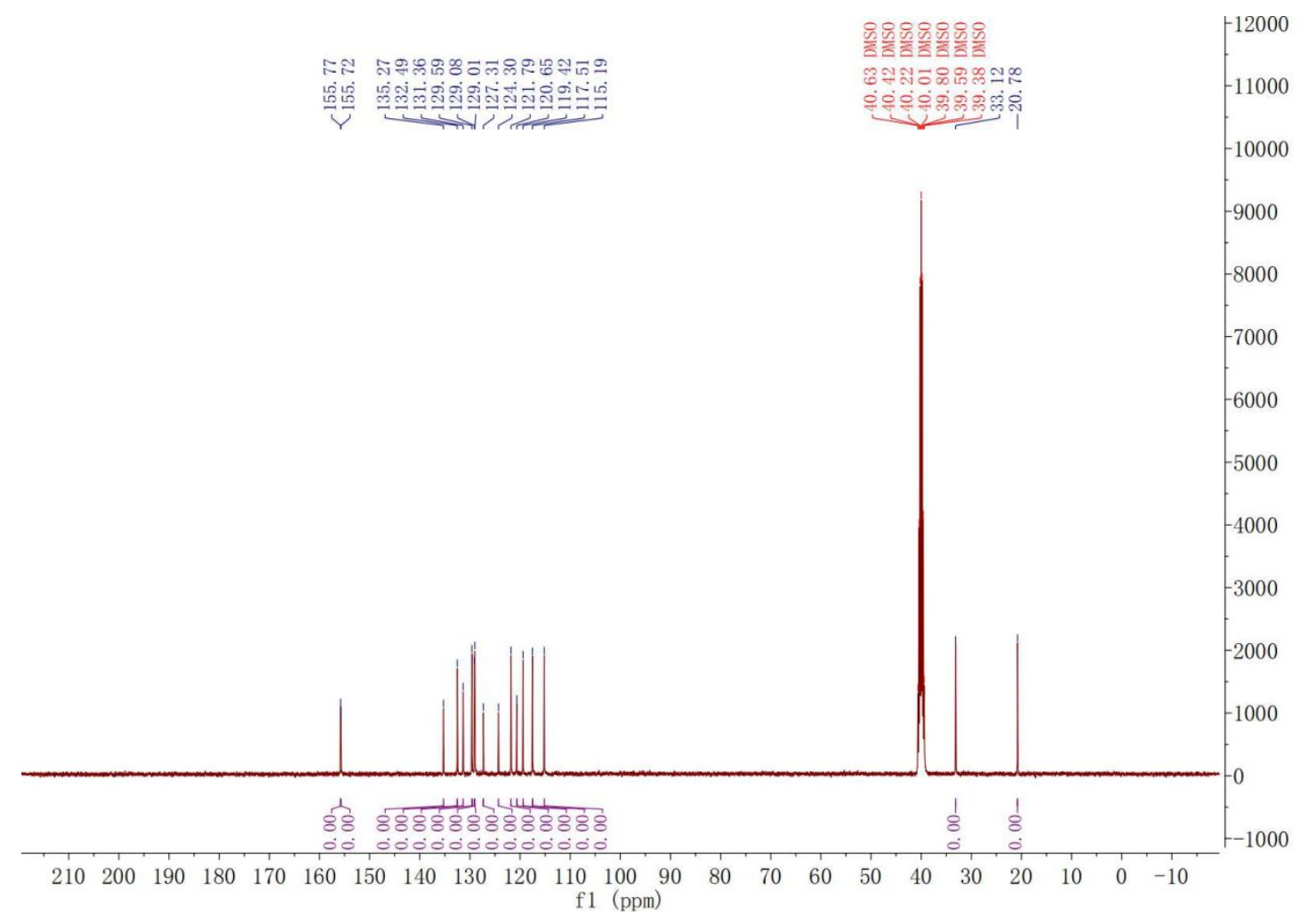

The ${ }^{13} \mathrm{C}$ NMR spectrogram of compound 2 


\section{Generic Display Report}

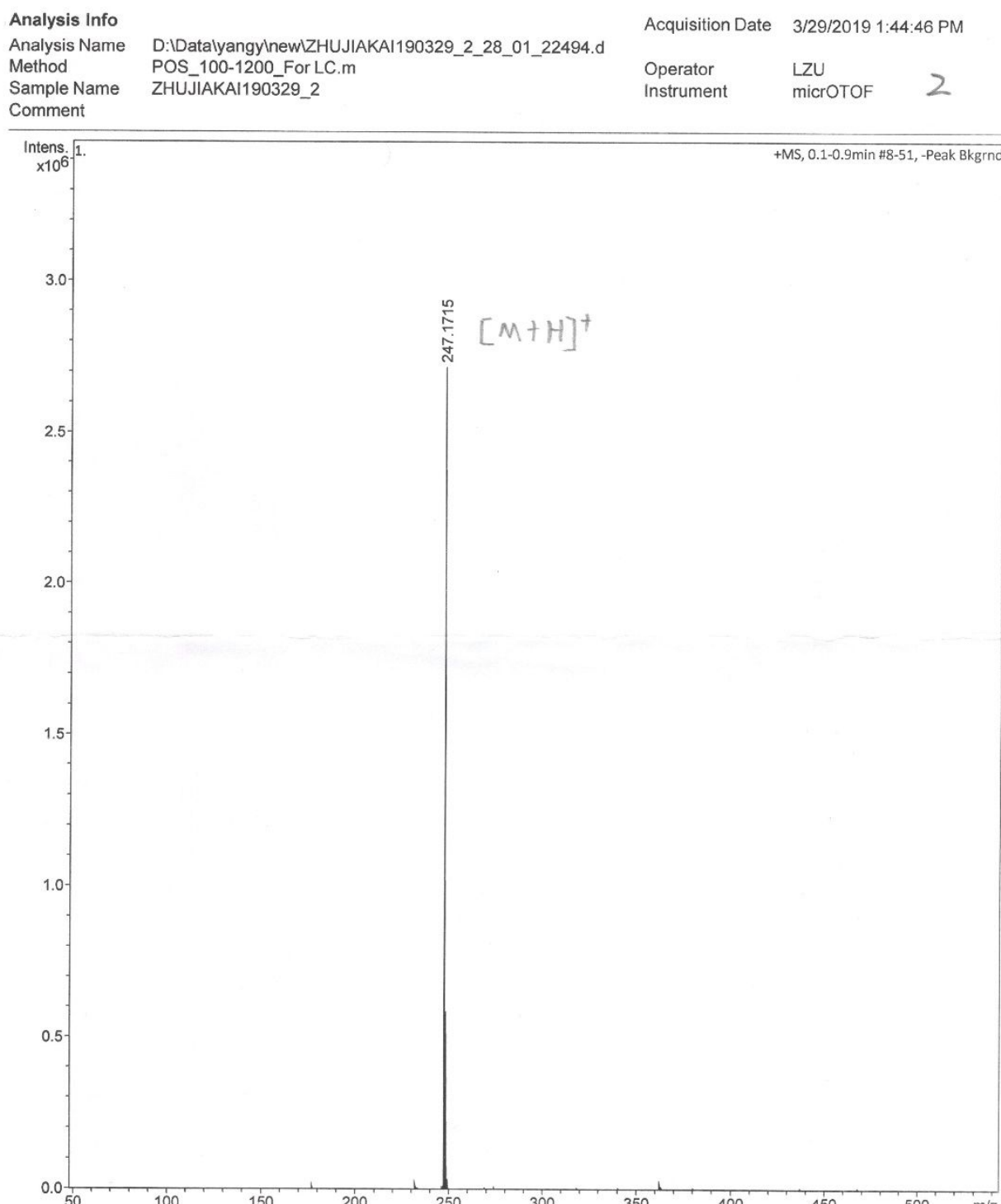

Bruker Compass DataAnalysis 4.1 printed: $3 / 29 / 2019$ 4:13:44 PM $\quad$ by: LZU Page 1 of 1

The Mass spectrogram of compound 2 


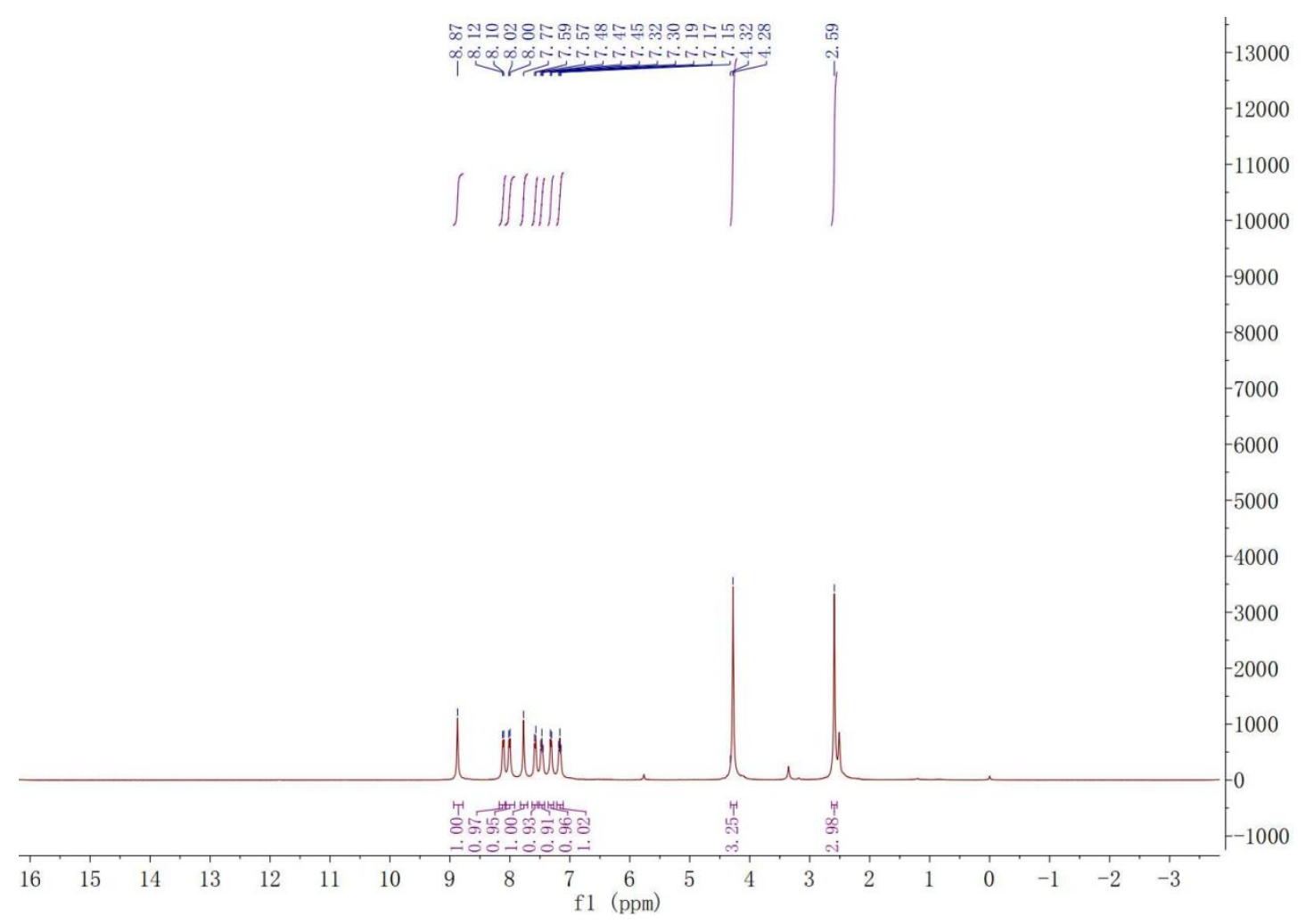

The ${ }^{1} \mathrm{H}$ NMR spectrogram of compound 7

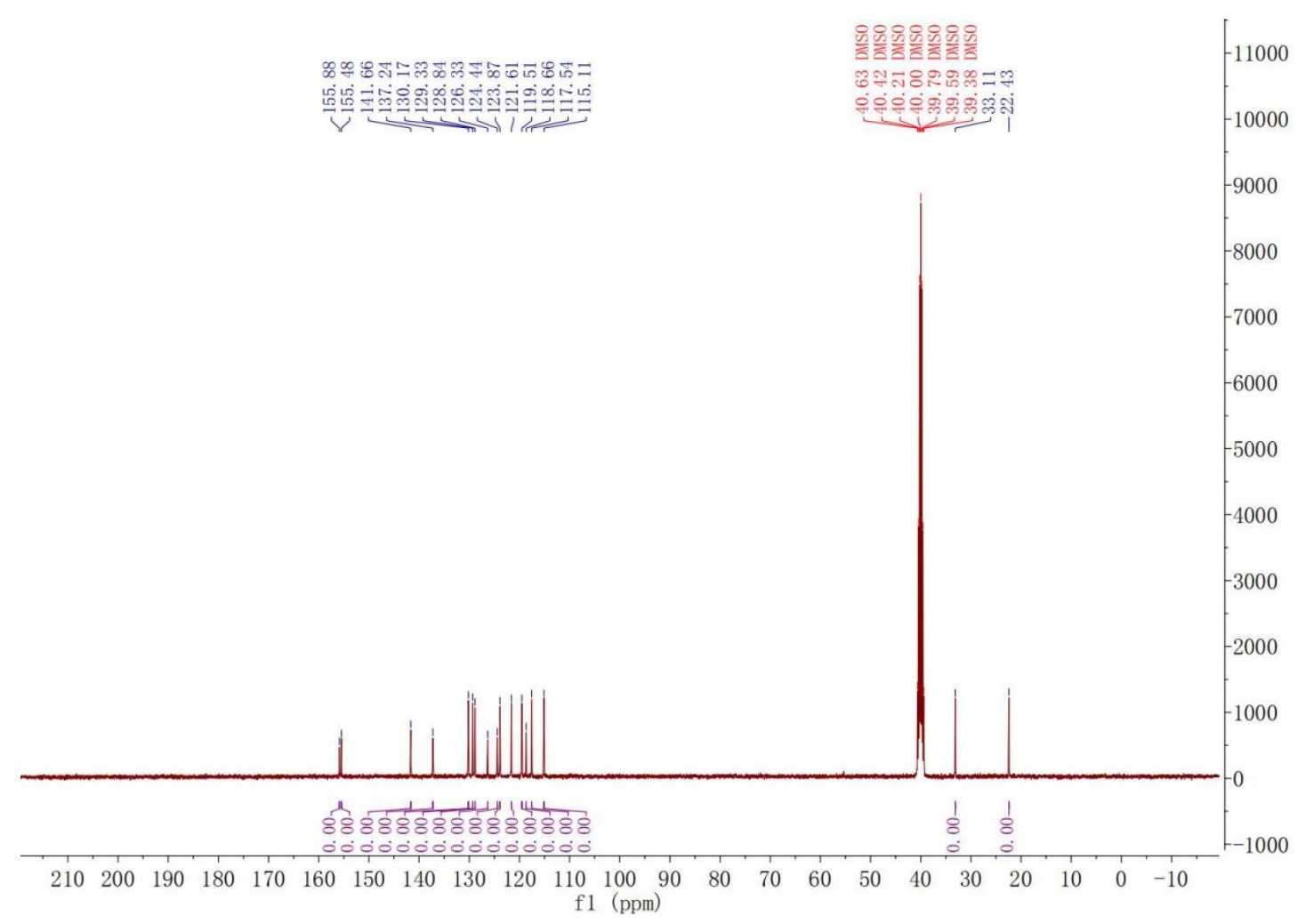

The ${ }^{13} \mathrm{C}$ NMR spectrogram of compound 7 


\section{Generic Display Report}

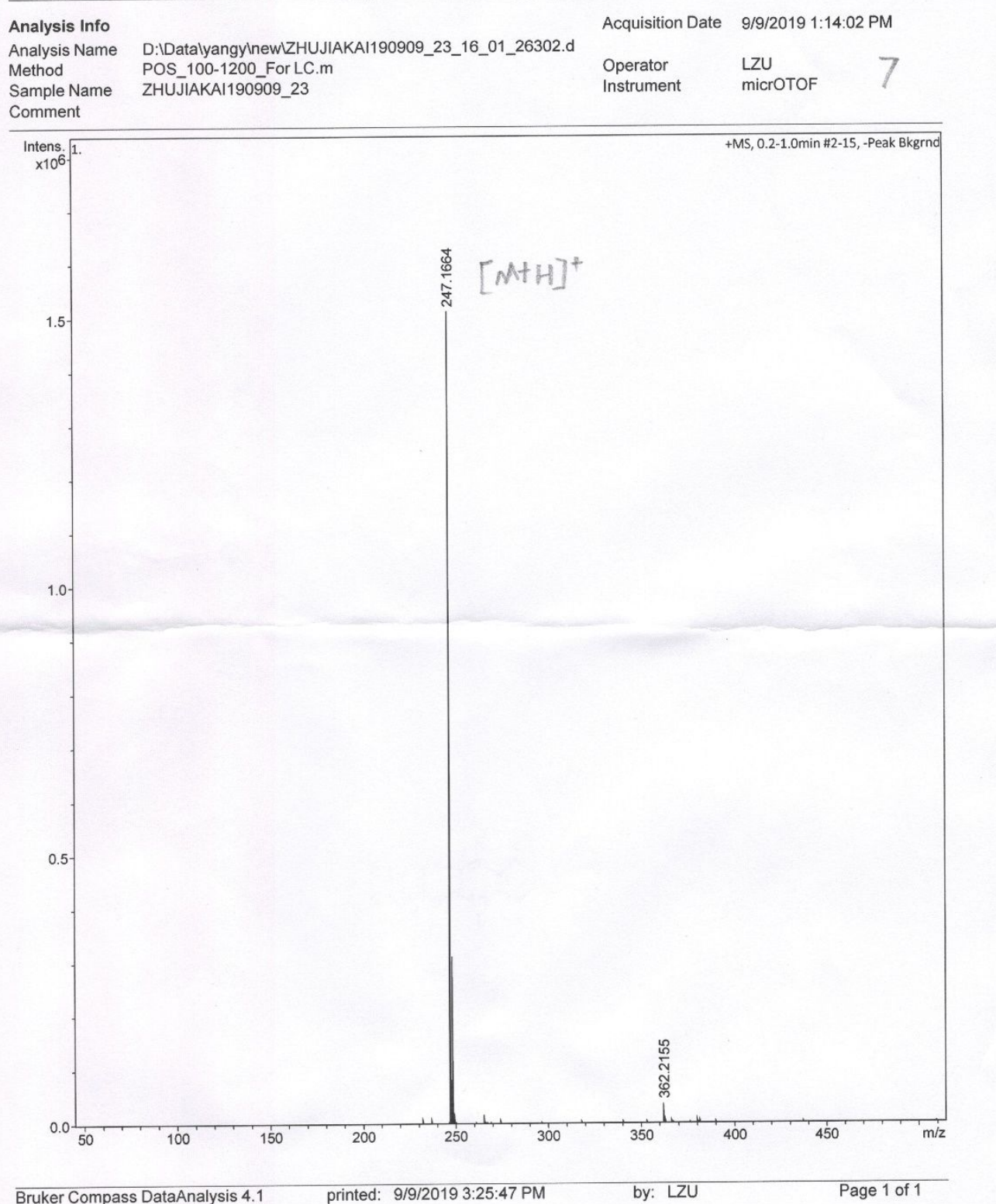

The Mass spectrogram of compound 7 


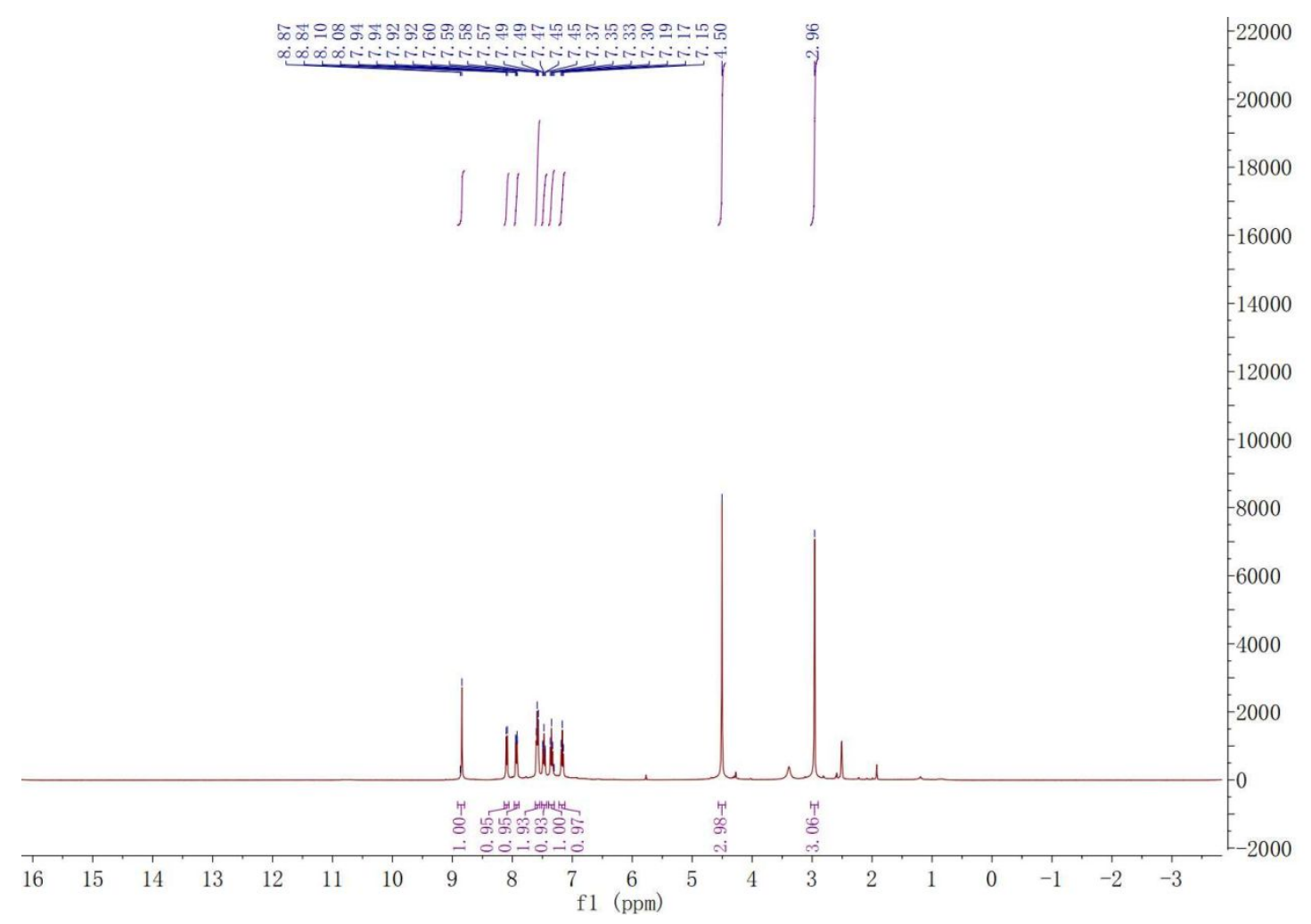

The ${ }^{1} \mathrm{H}$ NMR spectrogram of compound 12

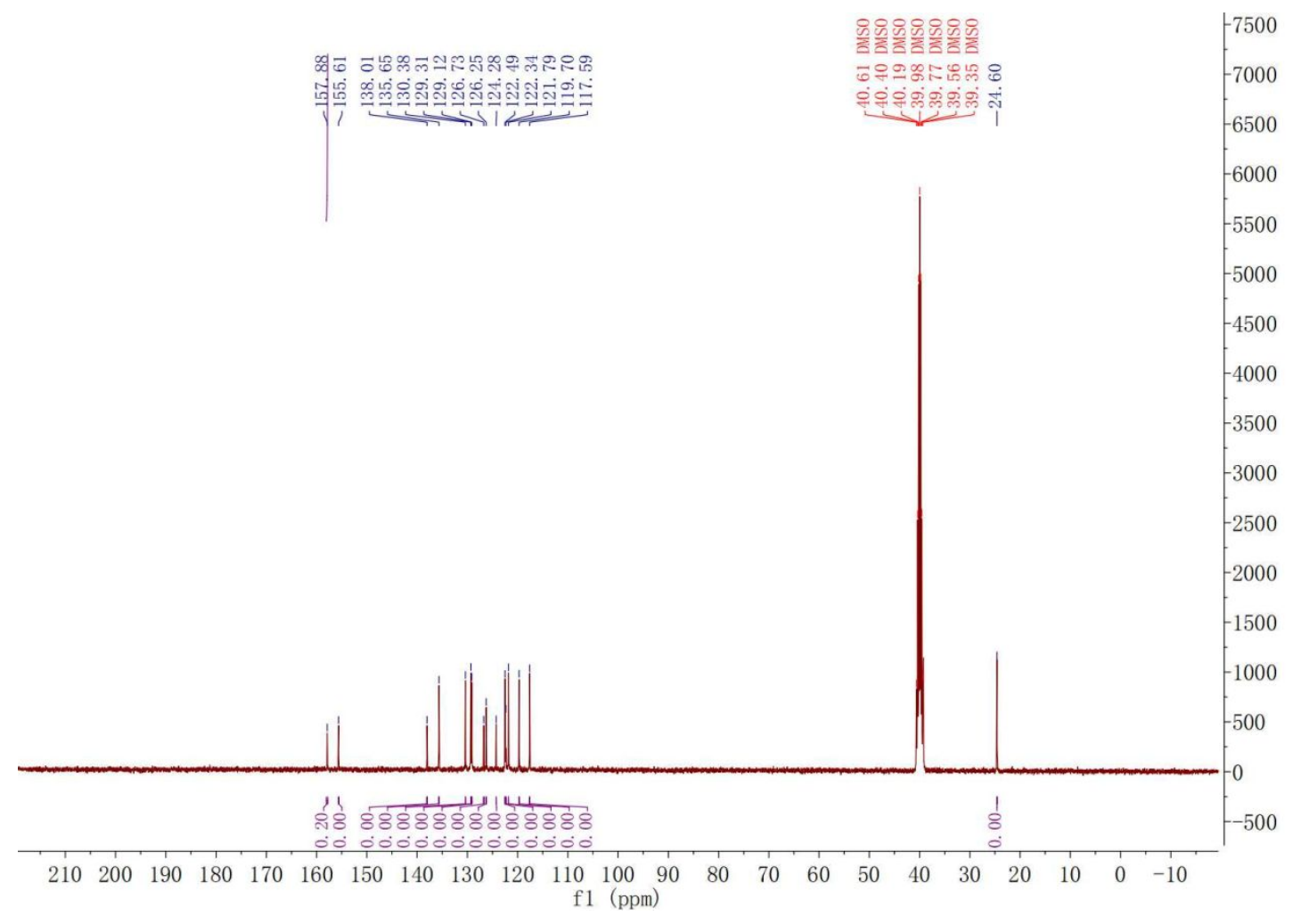

The ${ }^{13} \mathrm{C}$ NMR spectrogram of compound $\mathbf{1 2}$ 


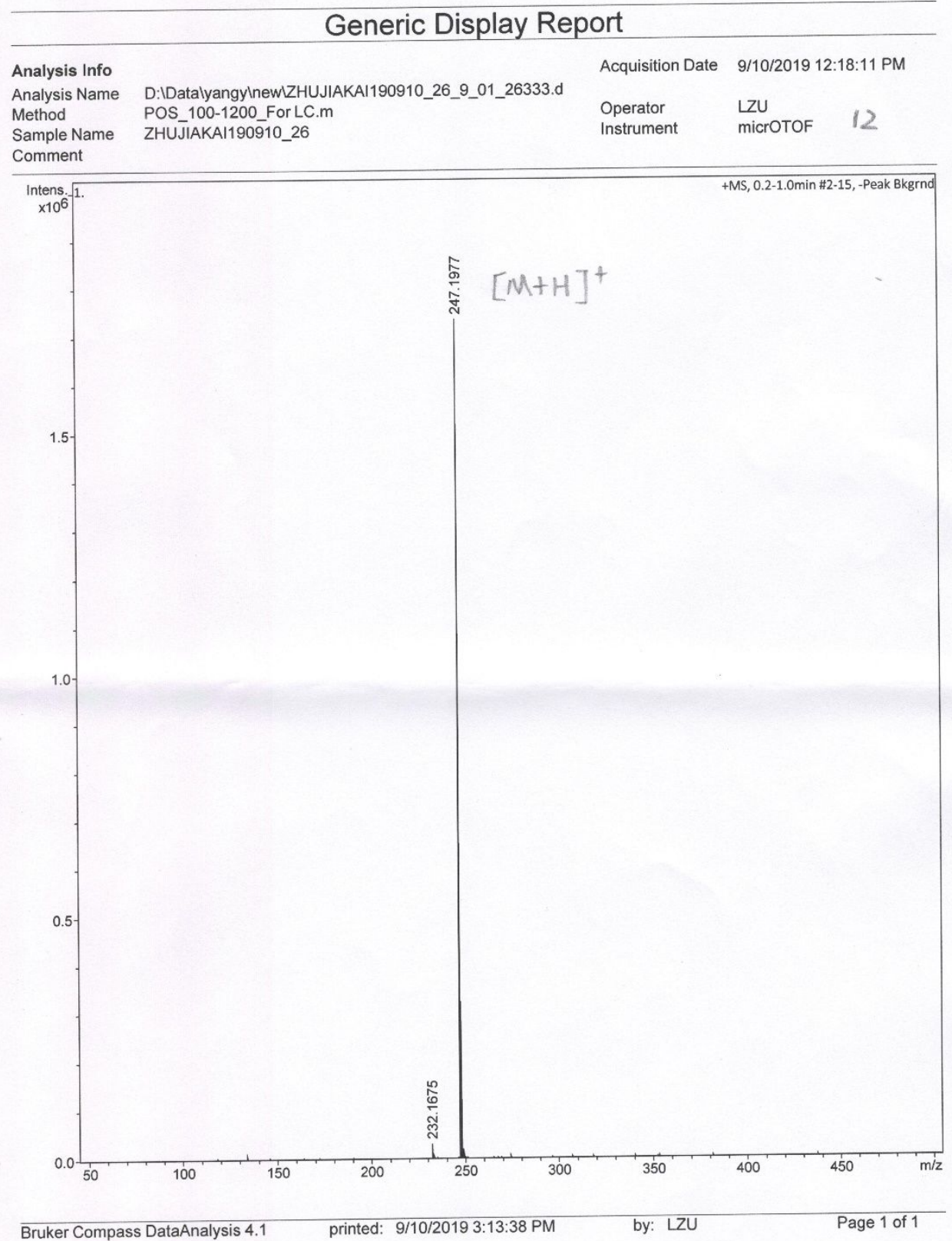

The Mass spectrogram of compound $\mathbf{1 2}$ 


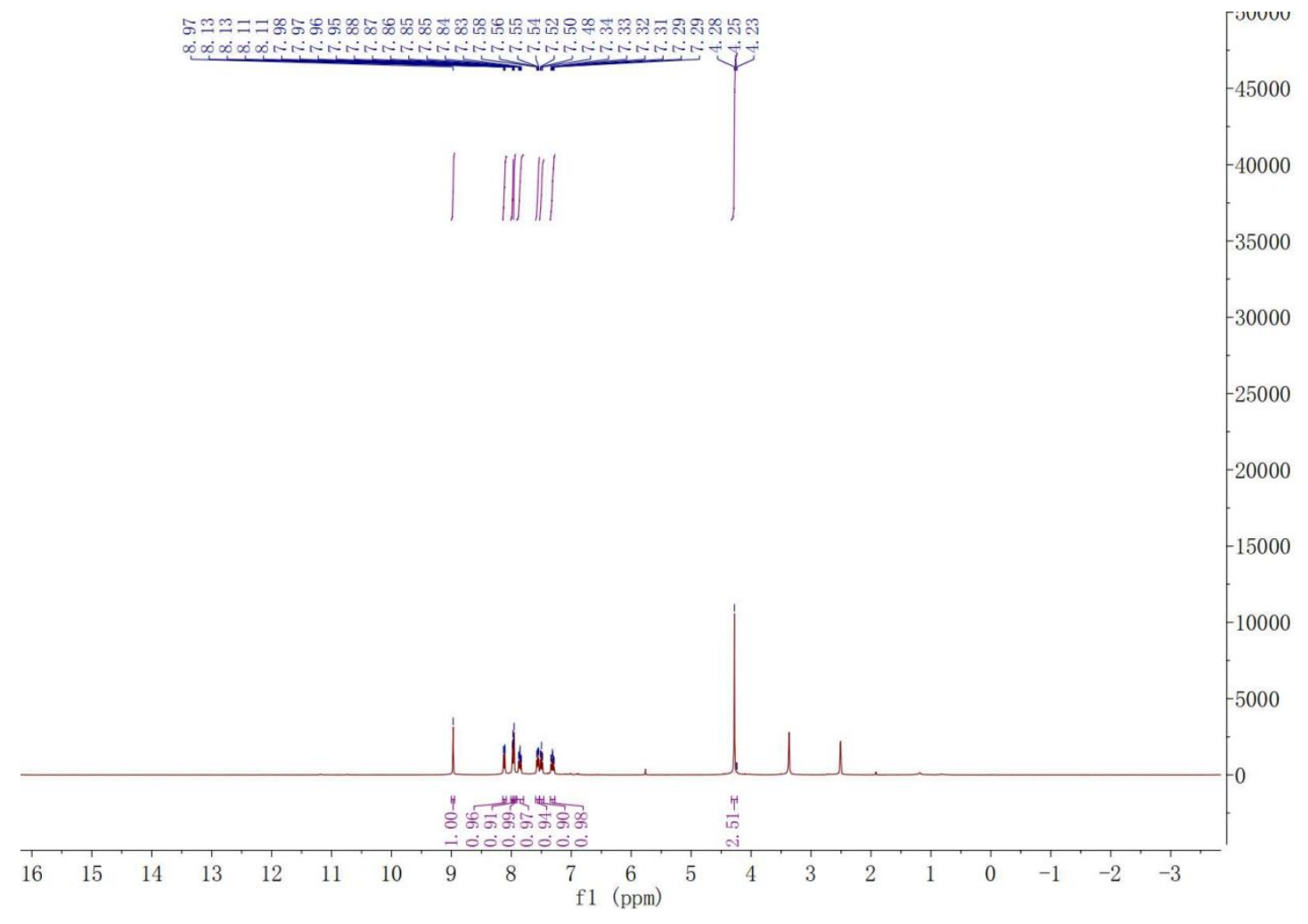

The ${ }^{1} \mathrm{H}$ NMR spectrogram of compound $\mathbf{1 8}$

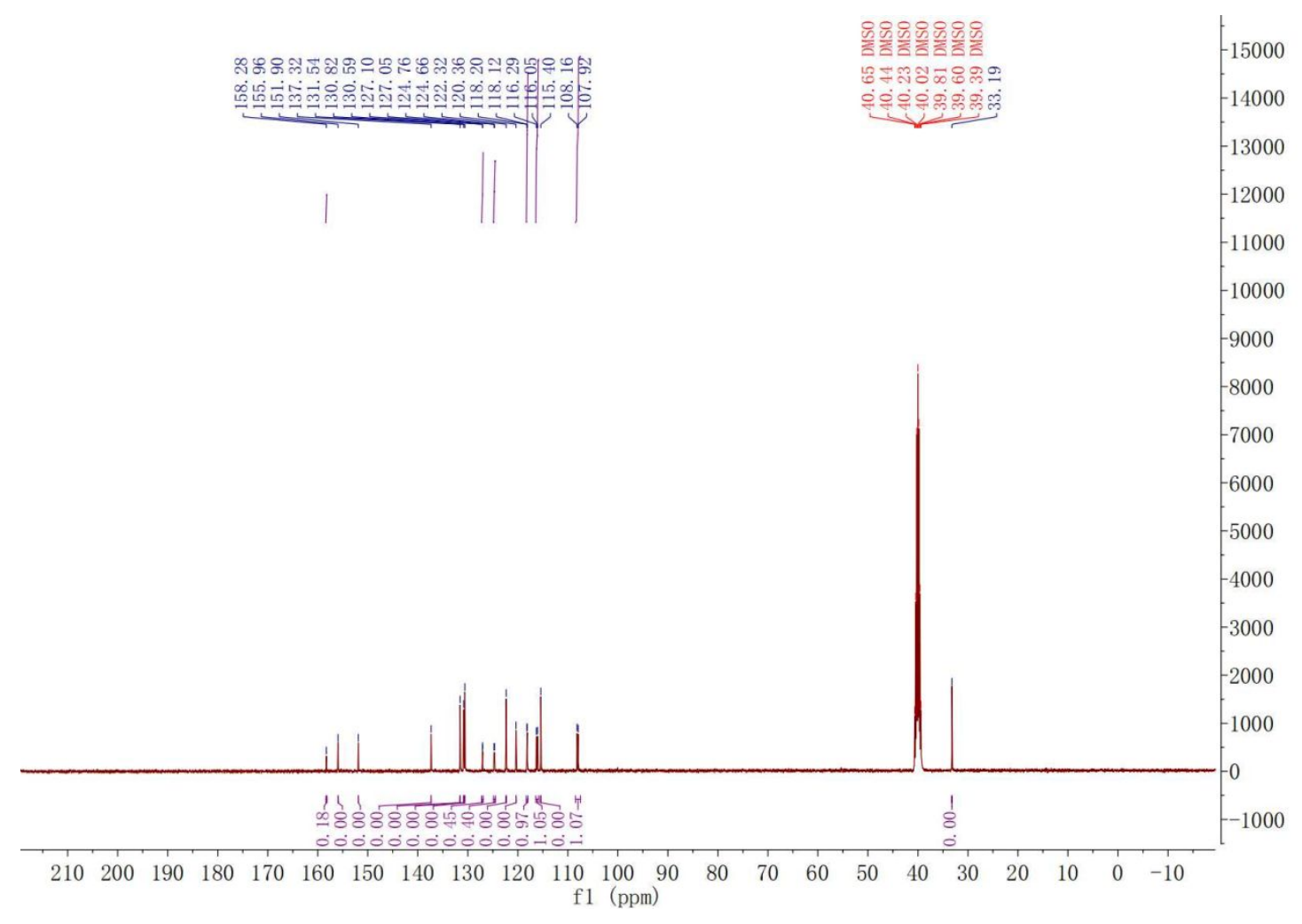

The ${ }^{13} \mathrm{C}$ NMR spectrogram of compound $\mathbf{1 8}$ 


\section{Generic Display Report}

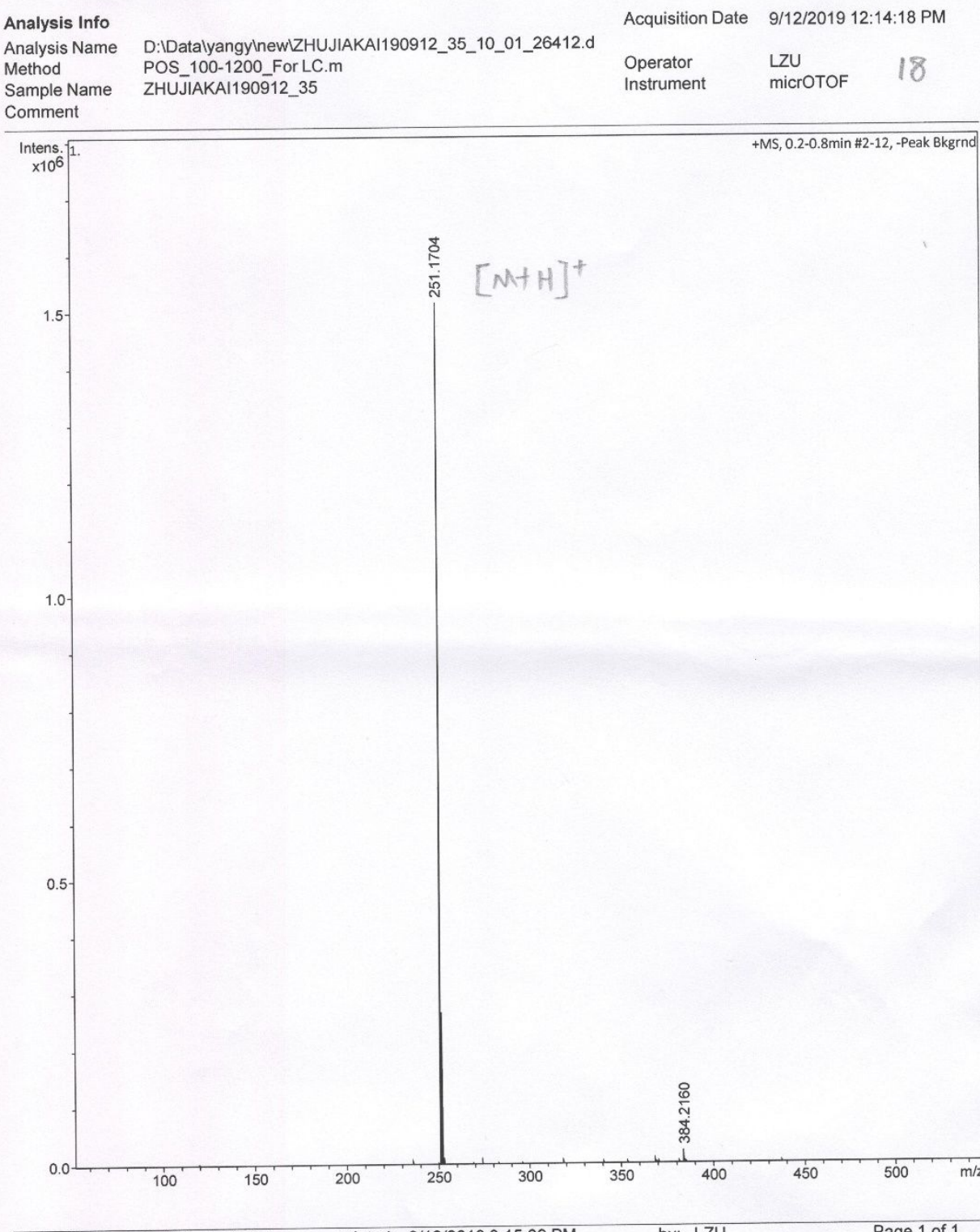

Bruker Compass DataAnalysis 4.1 printed: 9/12/2019 3:15:39 PM

by: LZU

Page 1 of 1

The Mass spectrogram of compound $\mathbf{1 8}$ 


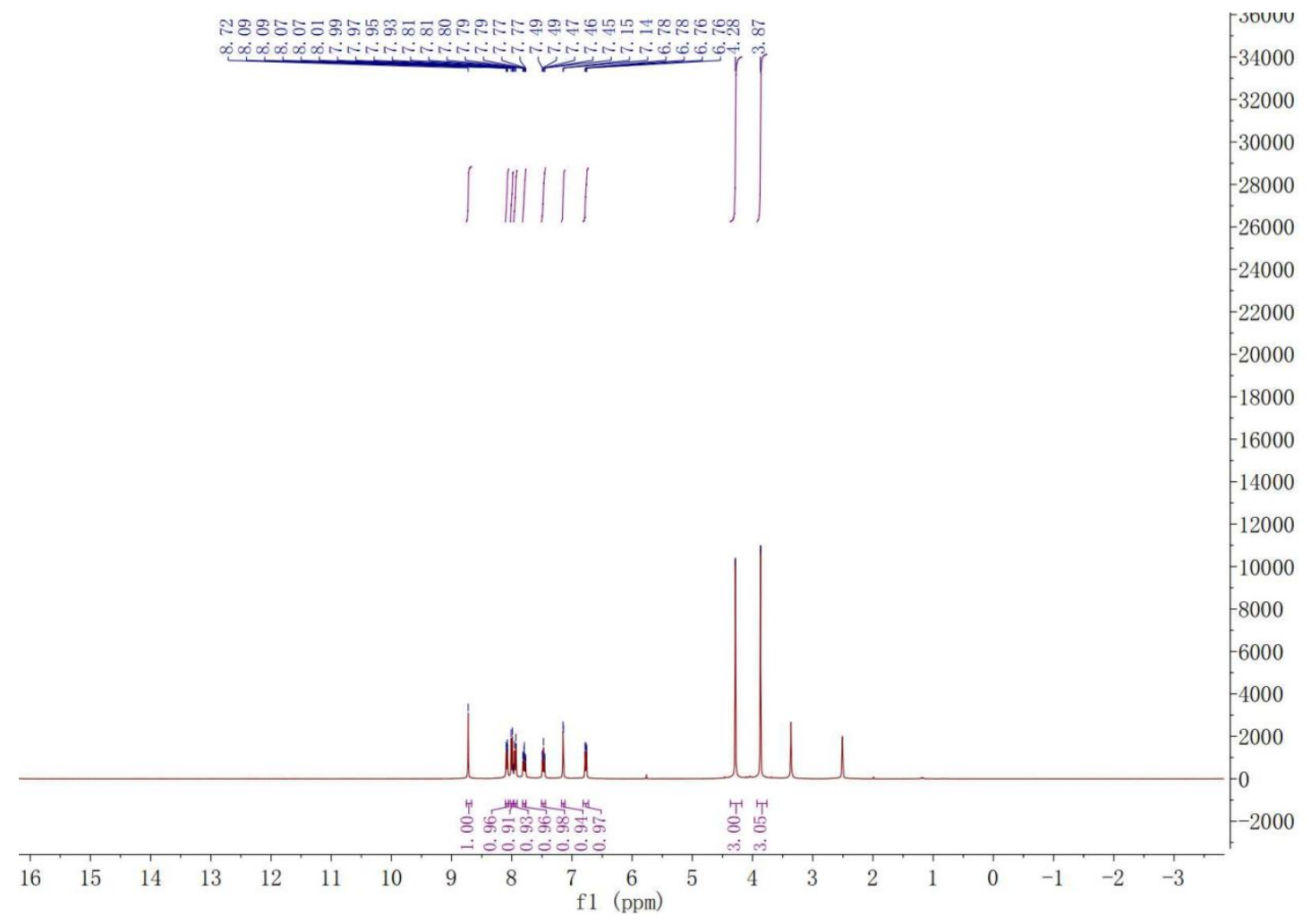

The ${ }^{1} \mathrm{H}$ NMR spectrogram of compound 22

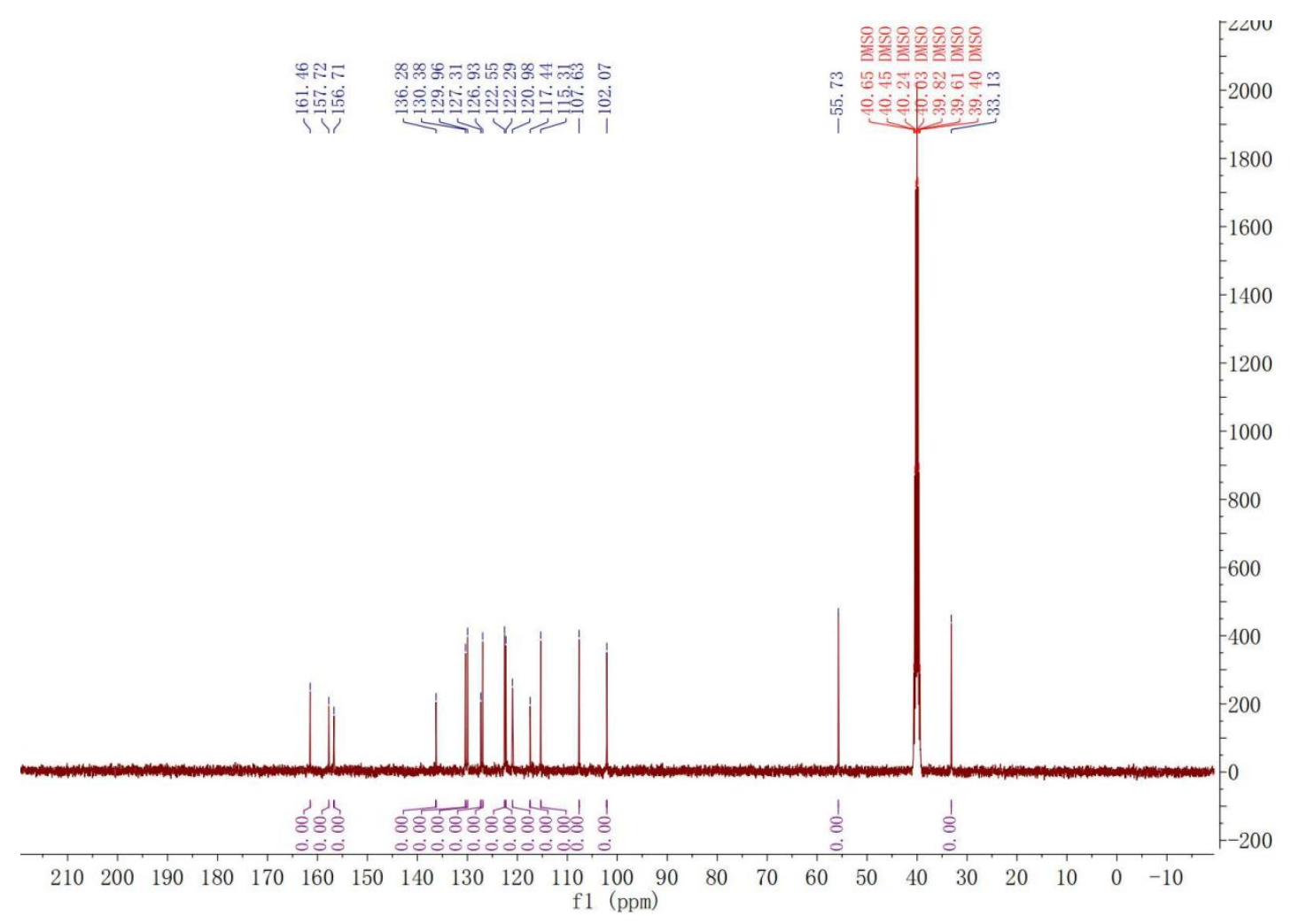

The ${ }^{13} \mathrm{C}$ NMR spectrogram of compound 22 


\section{Generic Display Report}

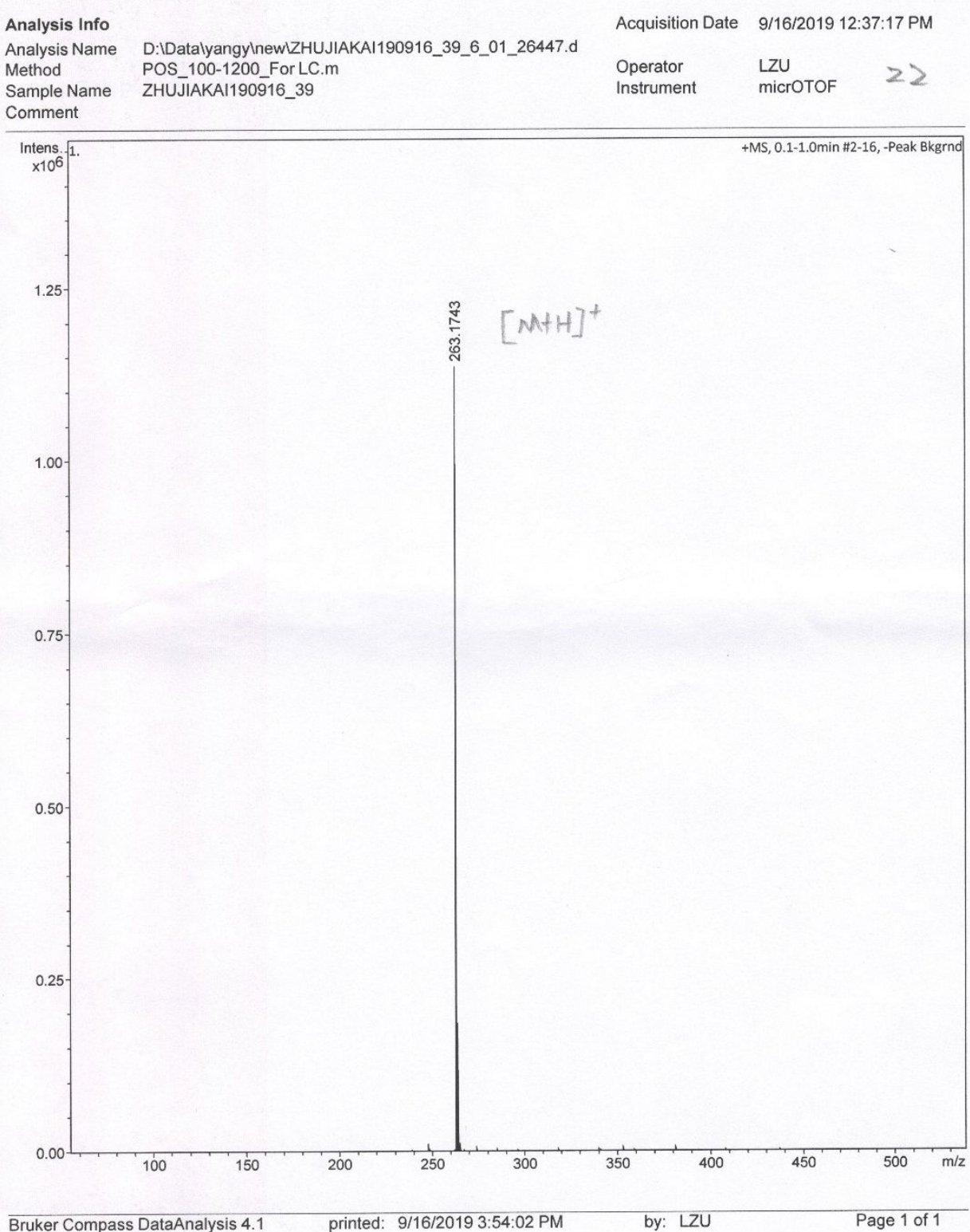

Bruker Compass DataAnalysis 4.1 printed: 9/16/2019 3:54:02 PM

by: LZU

Page 1 of 1

The Mass spectrogram of compound $\mathbf{2 2}$ 


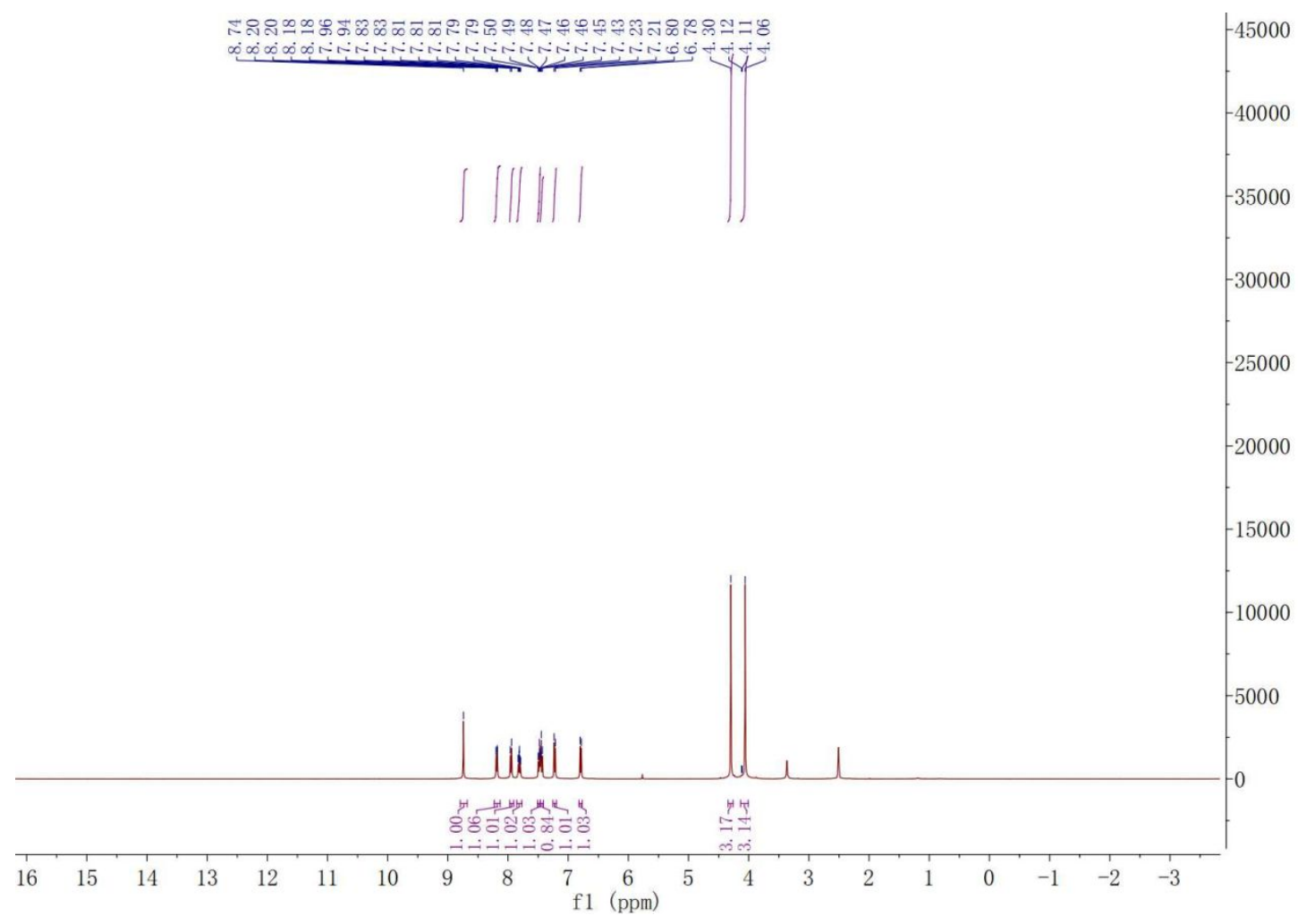

The ${ }^{1} \mathrm{H}$ NMR spectrogram of compound 27

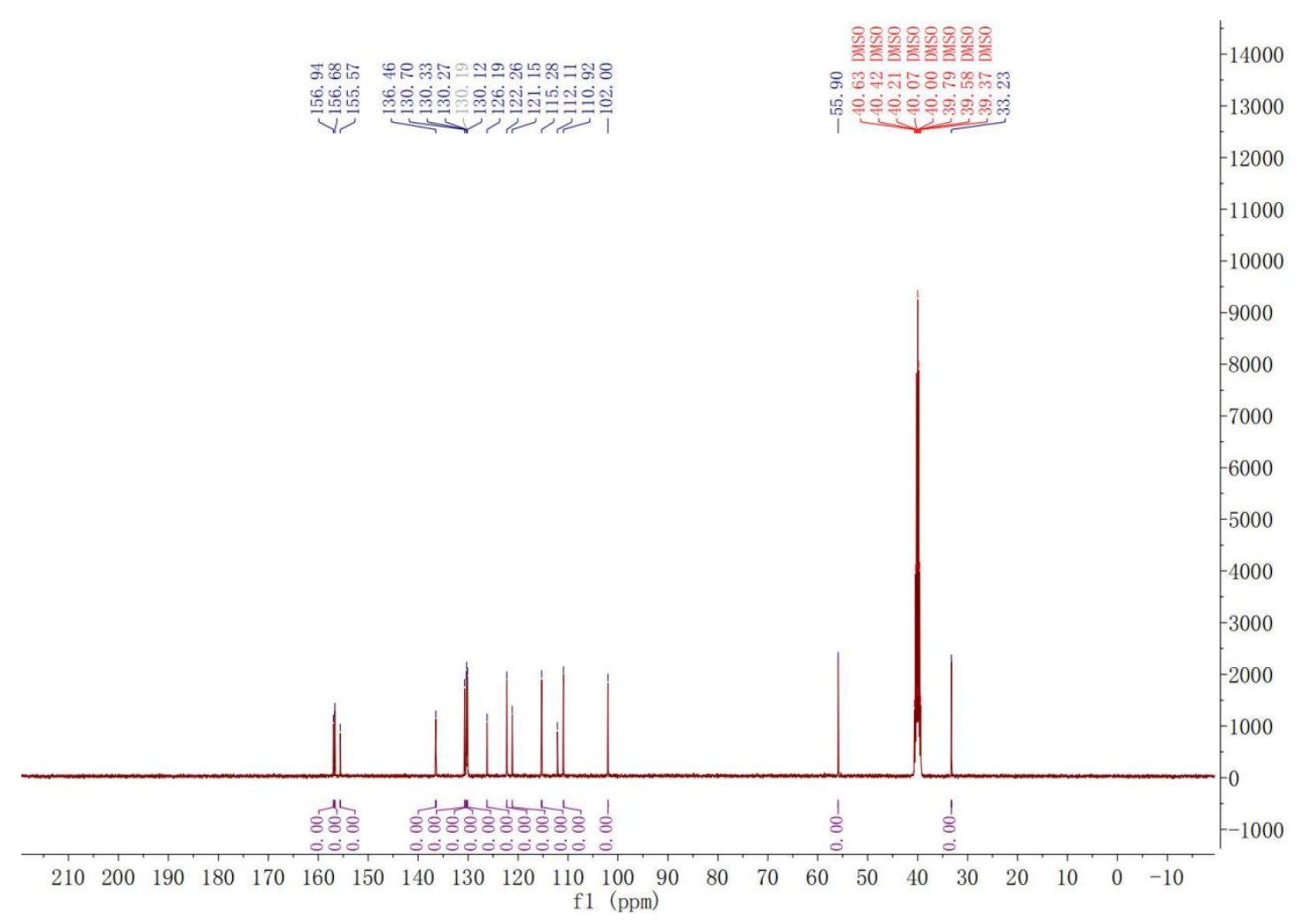

The ${ }^{13} \mathrm{C}$ NMR spectrogram of compound 27 


\section{Generic Display Report}

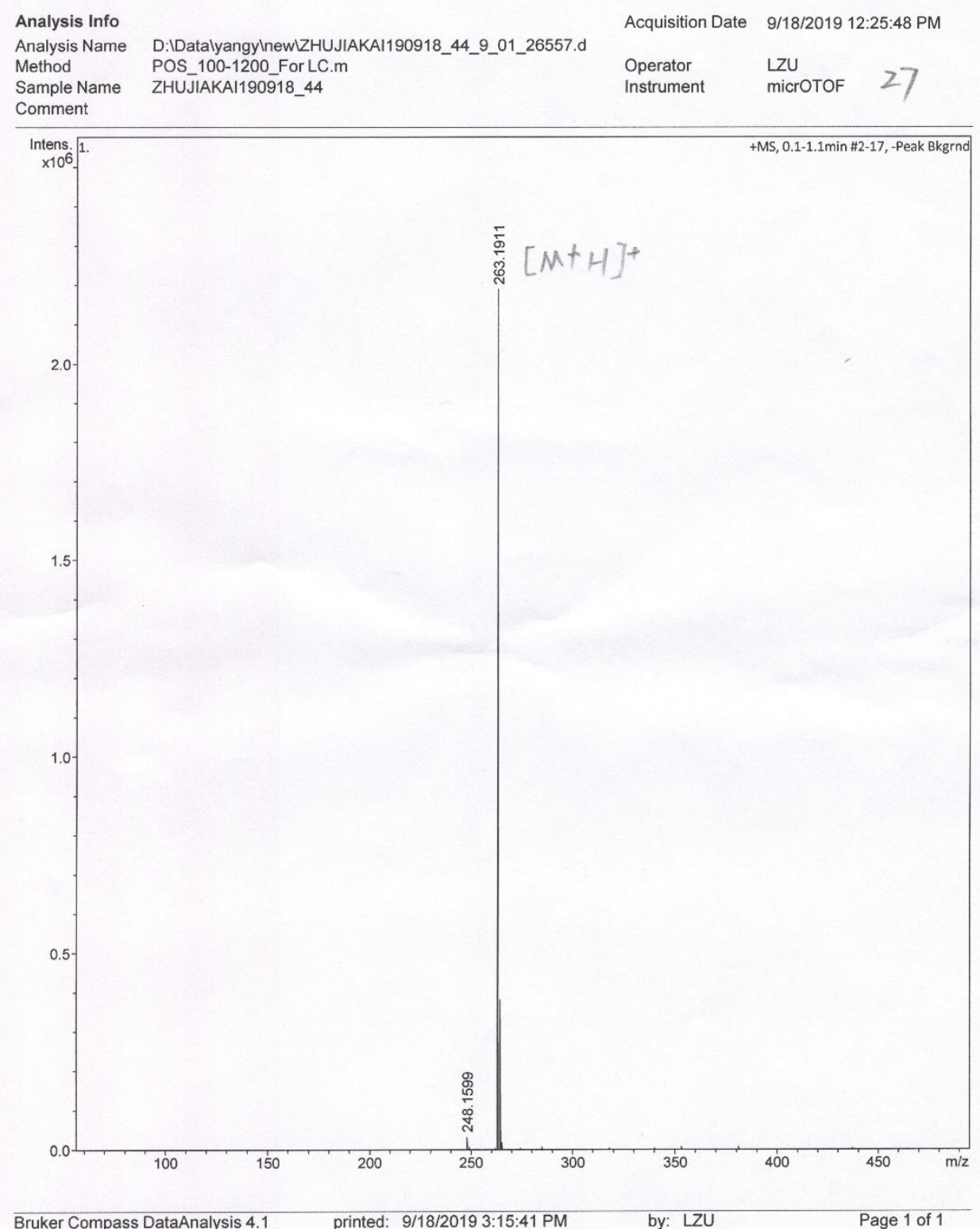

The Mass spectrogram of compound $\mathbf{2 7}$ 


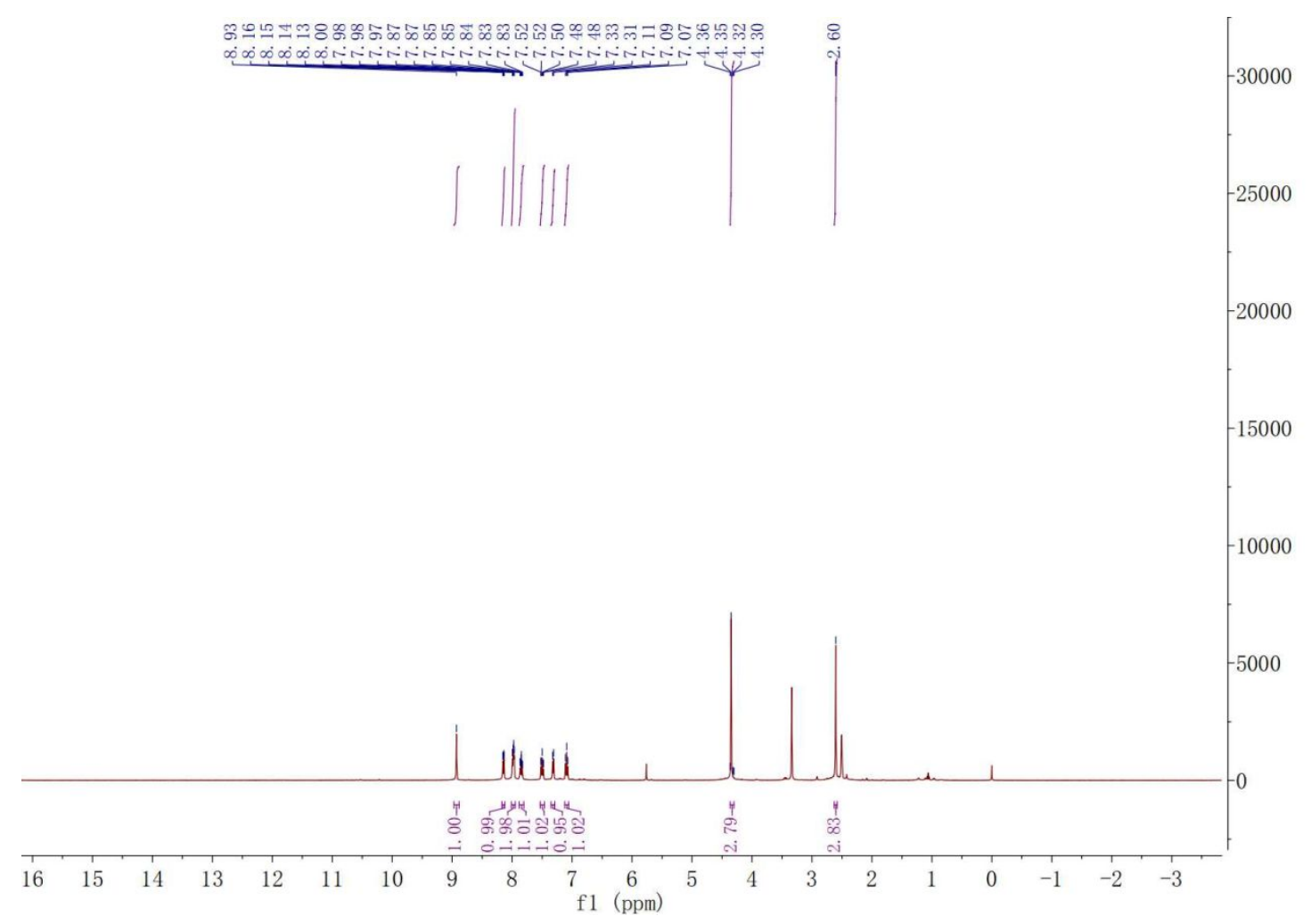

The ${ }^{1} \mathrm{H}$ NMR spectrogram of compound $\mathbf{3 1}$

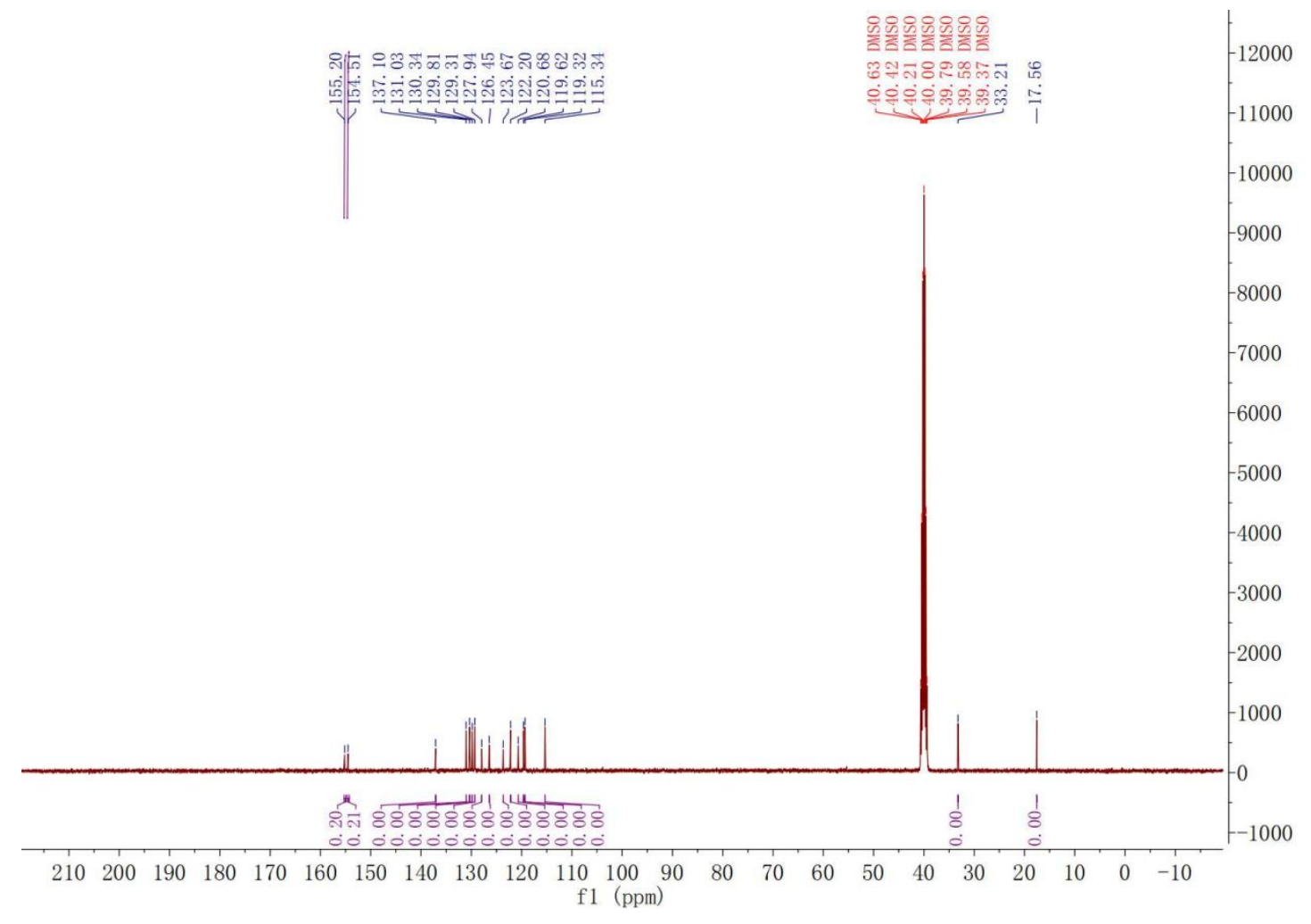

The ${ }^{13} \mathrm{C}$ NMR spectrogram of compound 31 


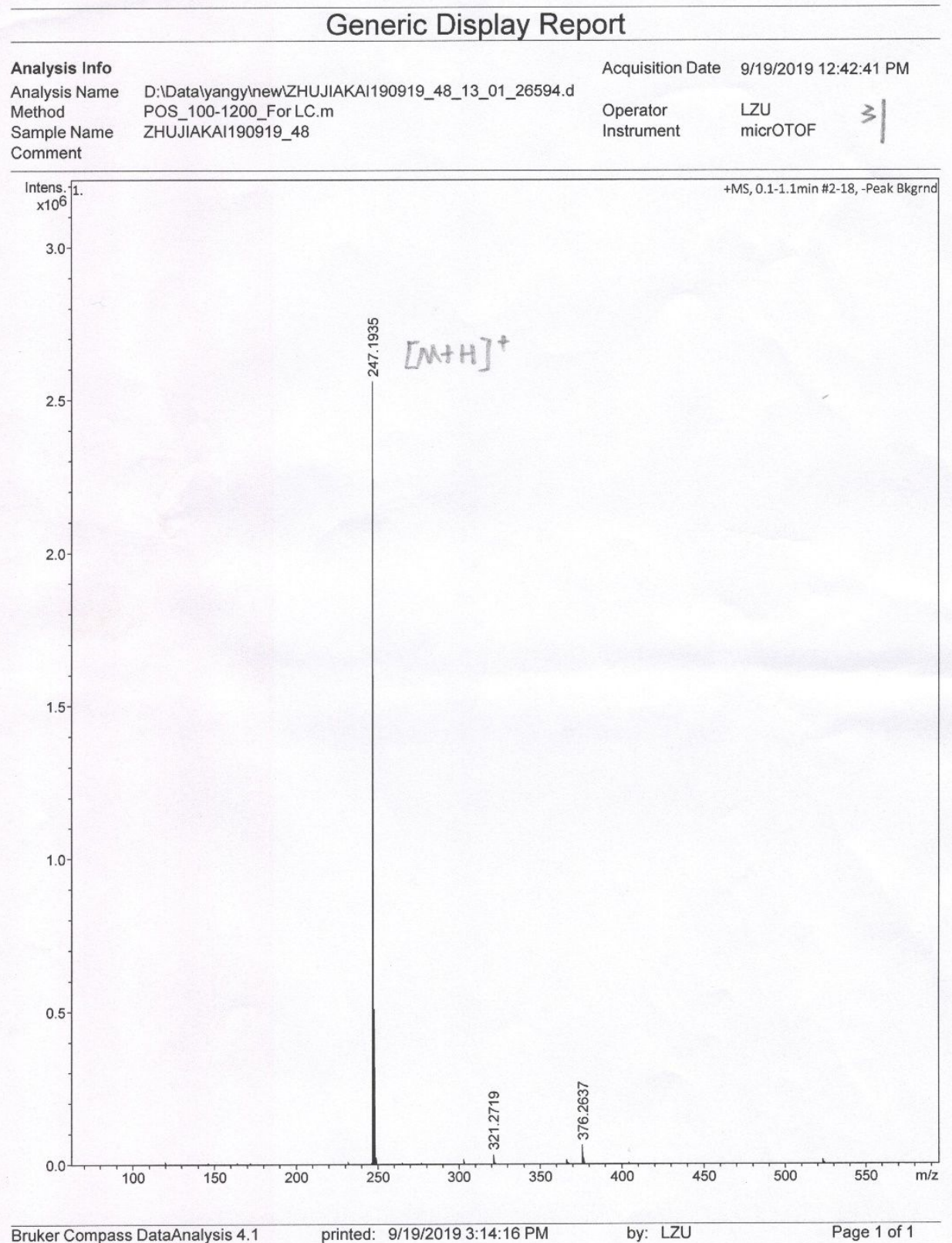

The Mass spectrogram of compound $\mathbf{3 1}$ 


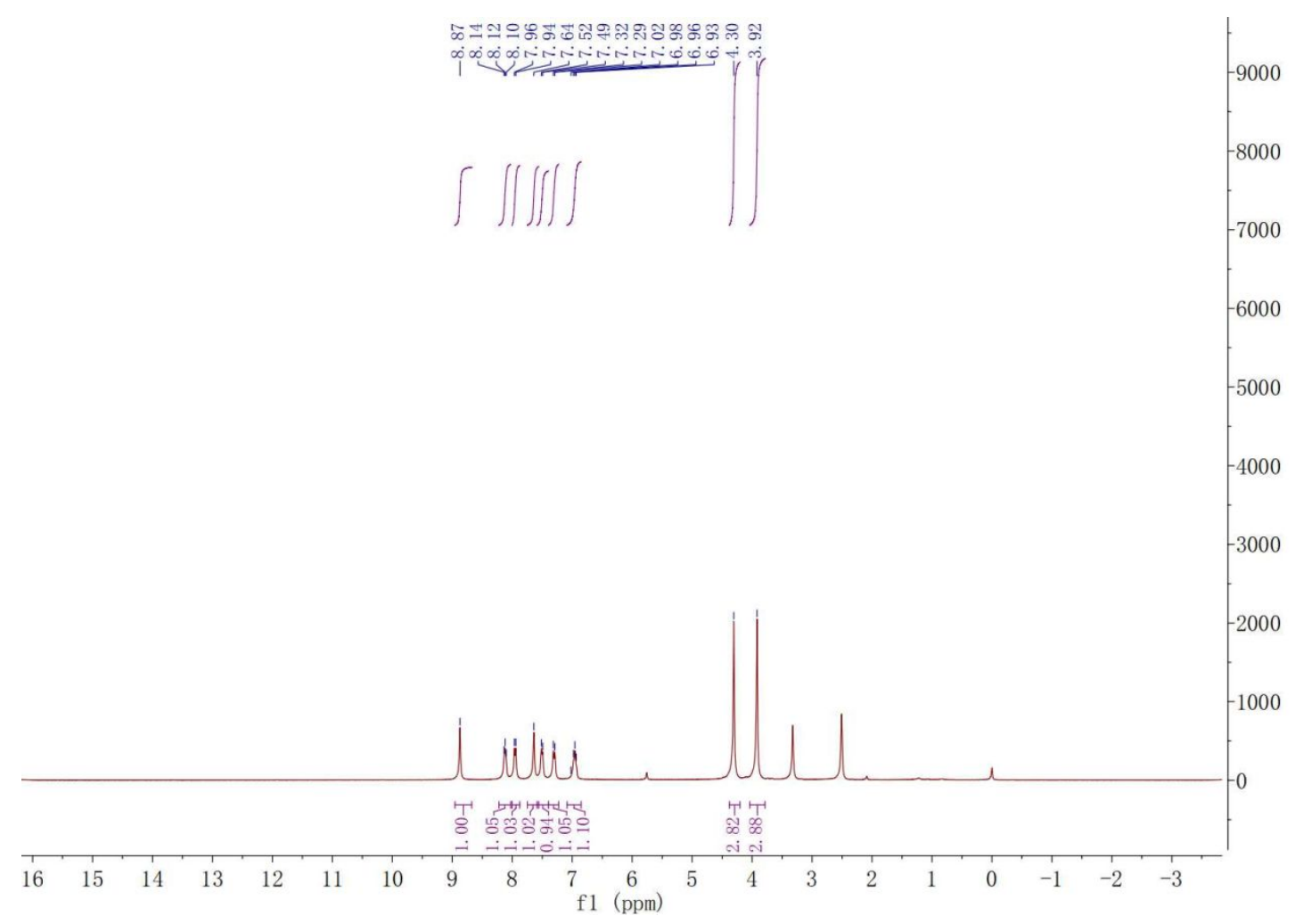

The ${ }^{1} \mathrm{H}$ NMR spectrogram of compound 35

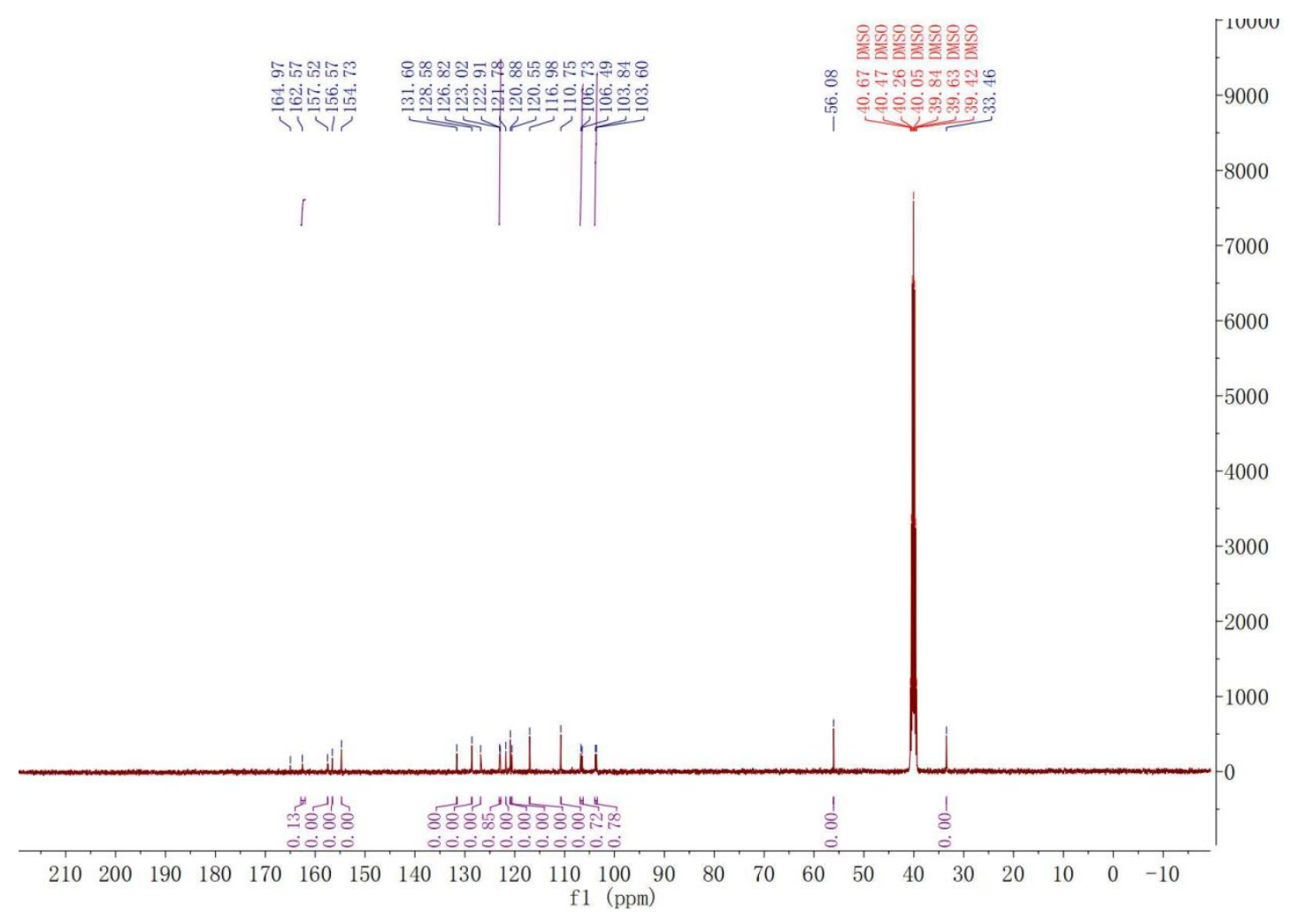

The ${ }^{13} \mathrm{C}$ NMR spectrogram of compound $\mathbf{3 5}$ 


\section{Generic Display Report}

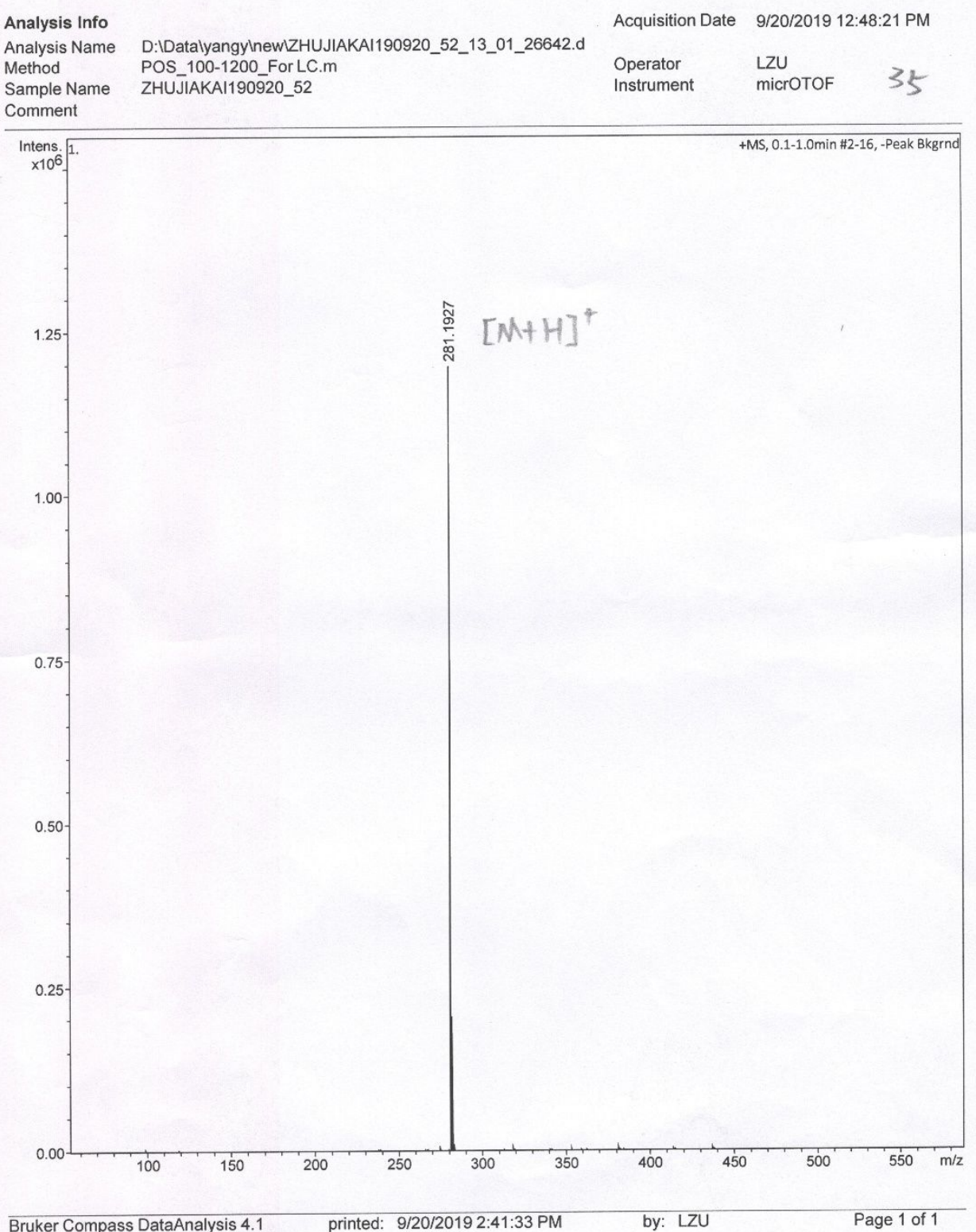

The Mass spectrogram of compound $\mathbf{3 5}$ 


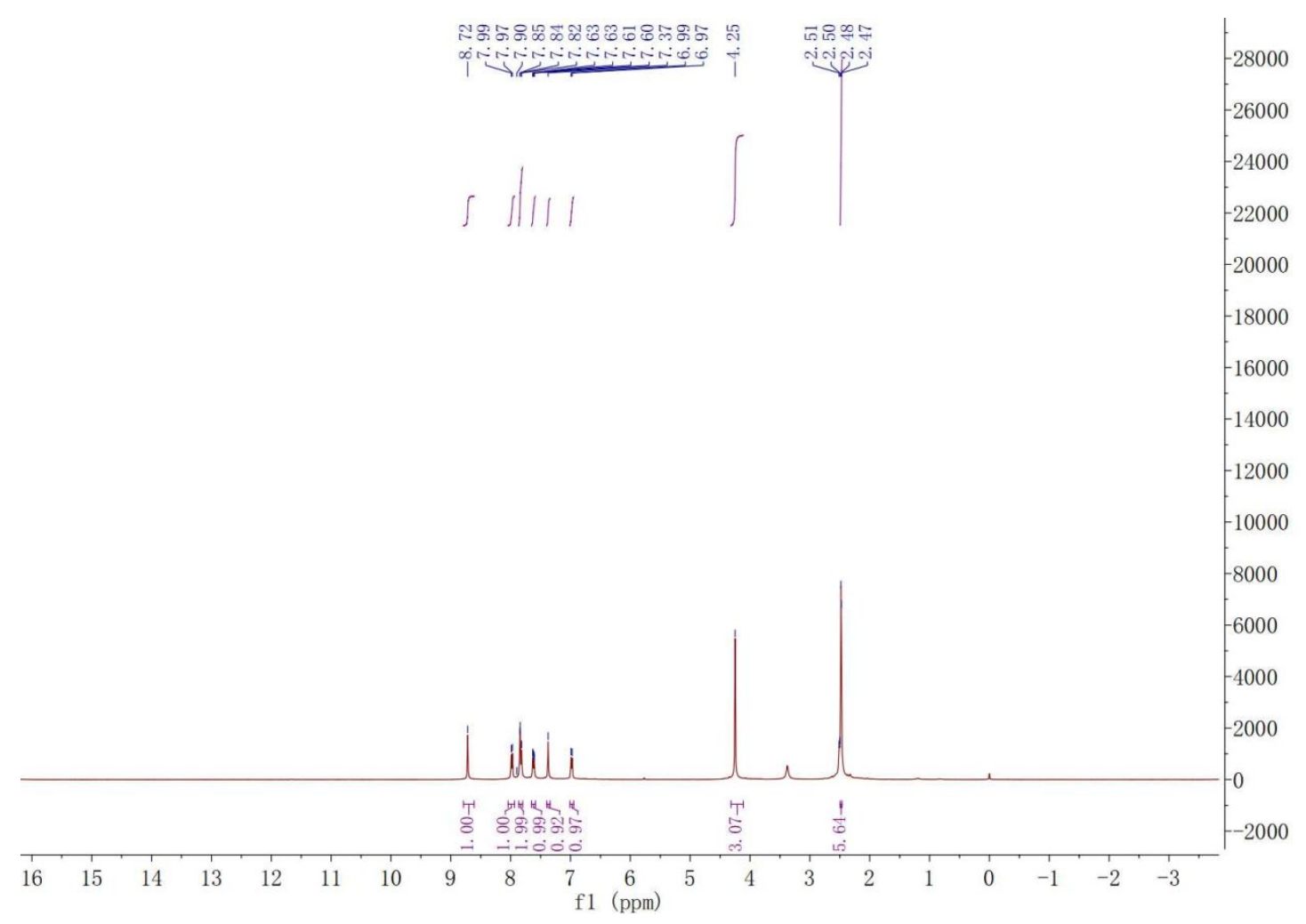

The ${ }^{1} \mathrm{H}$ NMR spectrogram of compound $\mathbf{4 3}$

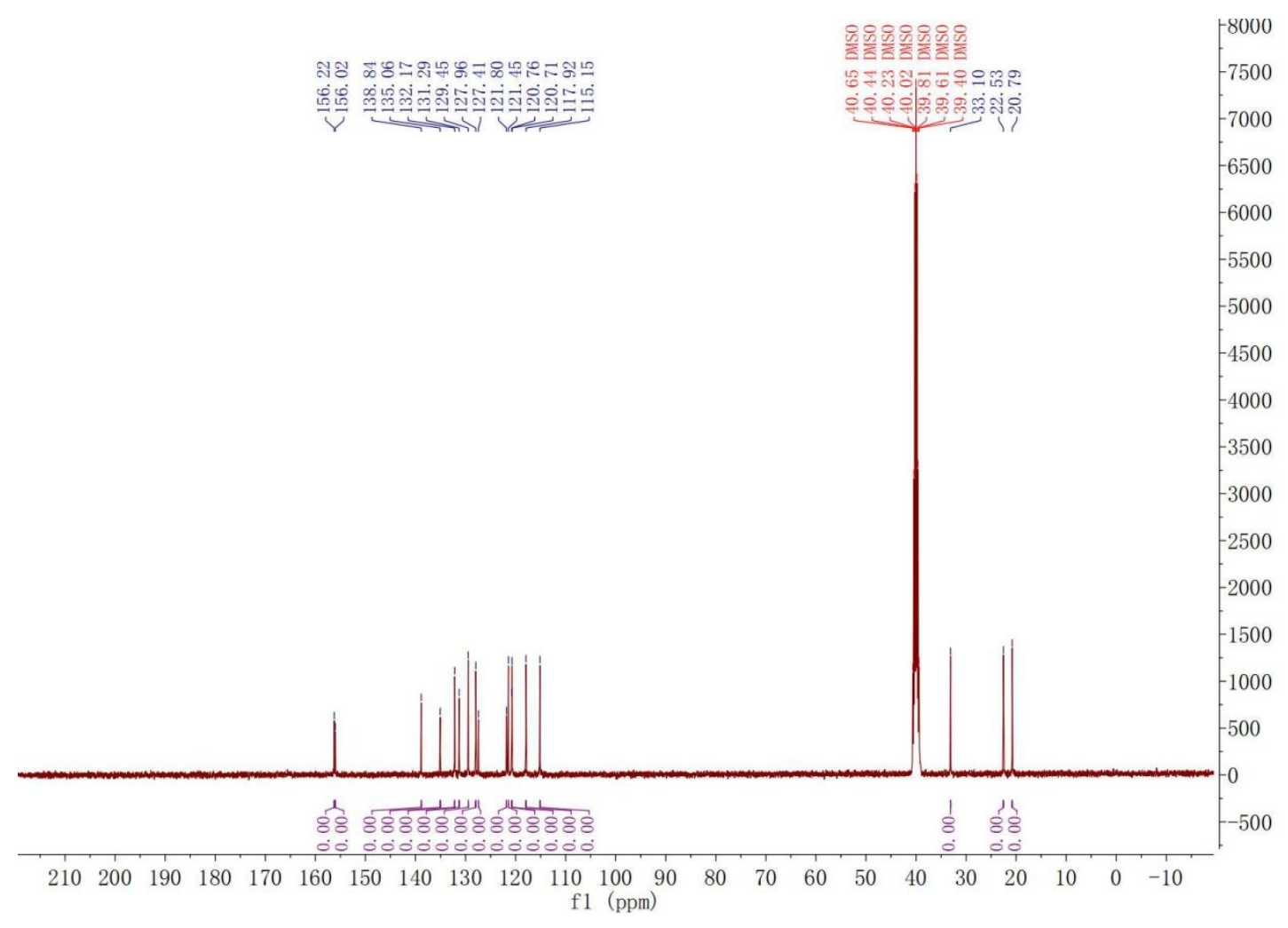

The ${ }^{13} \mathrm{C}$ NMR spectrogram of compound $\mathbf{4 3}$ 


\section{Generic Display Report}

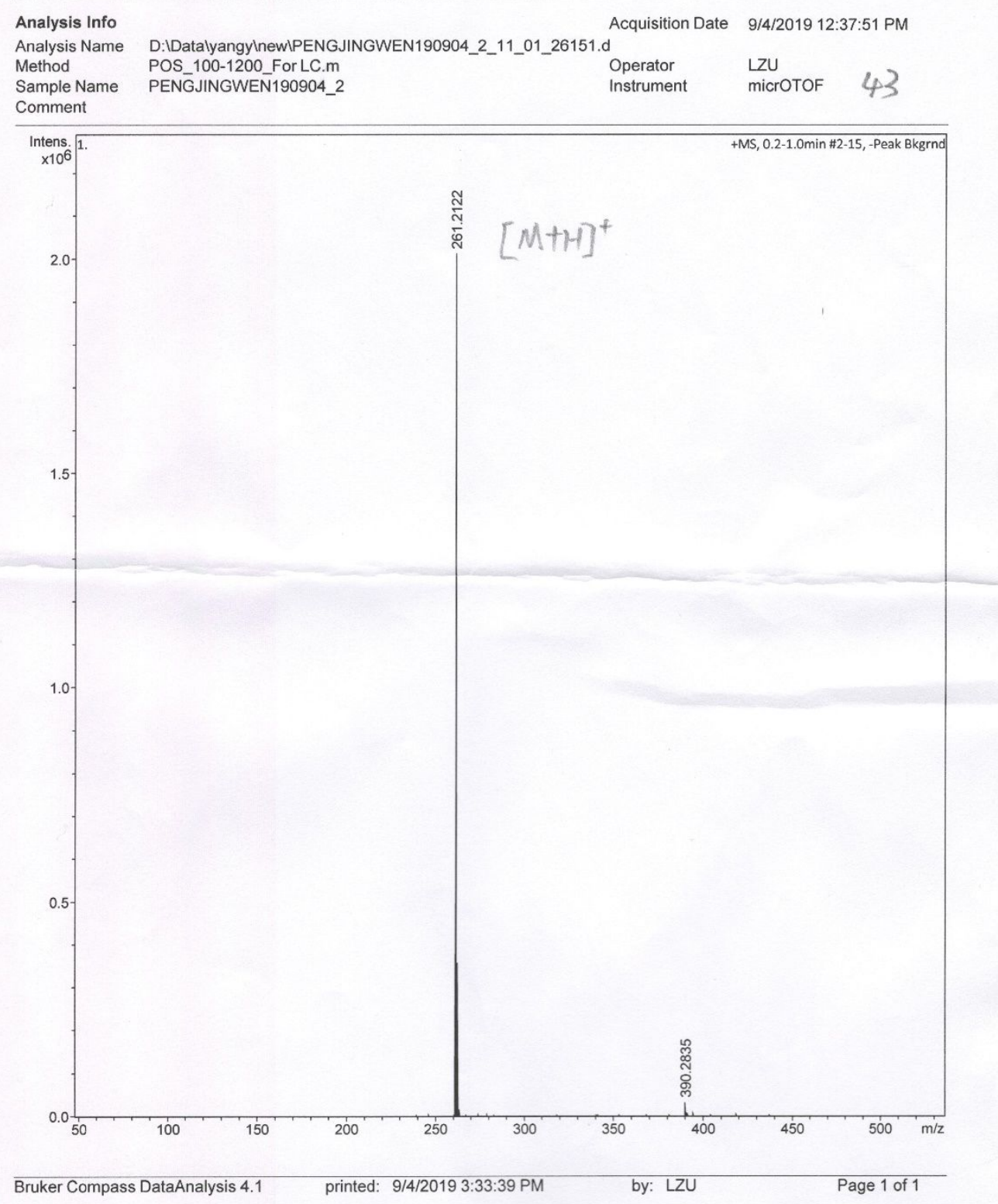

The Mass spectrogram of compound $\mathbf{4 3}$ 


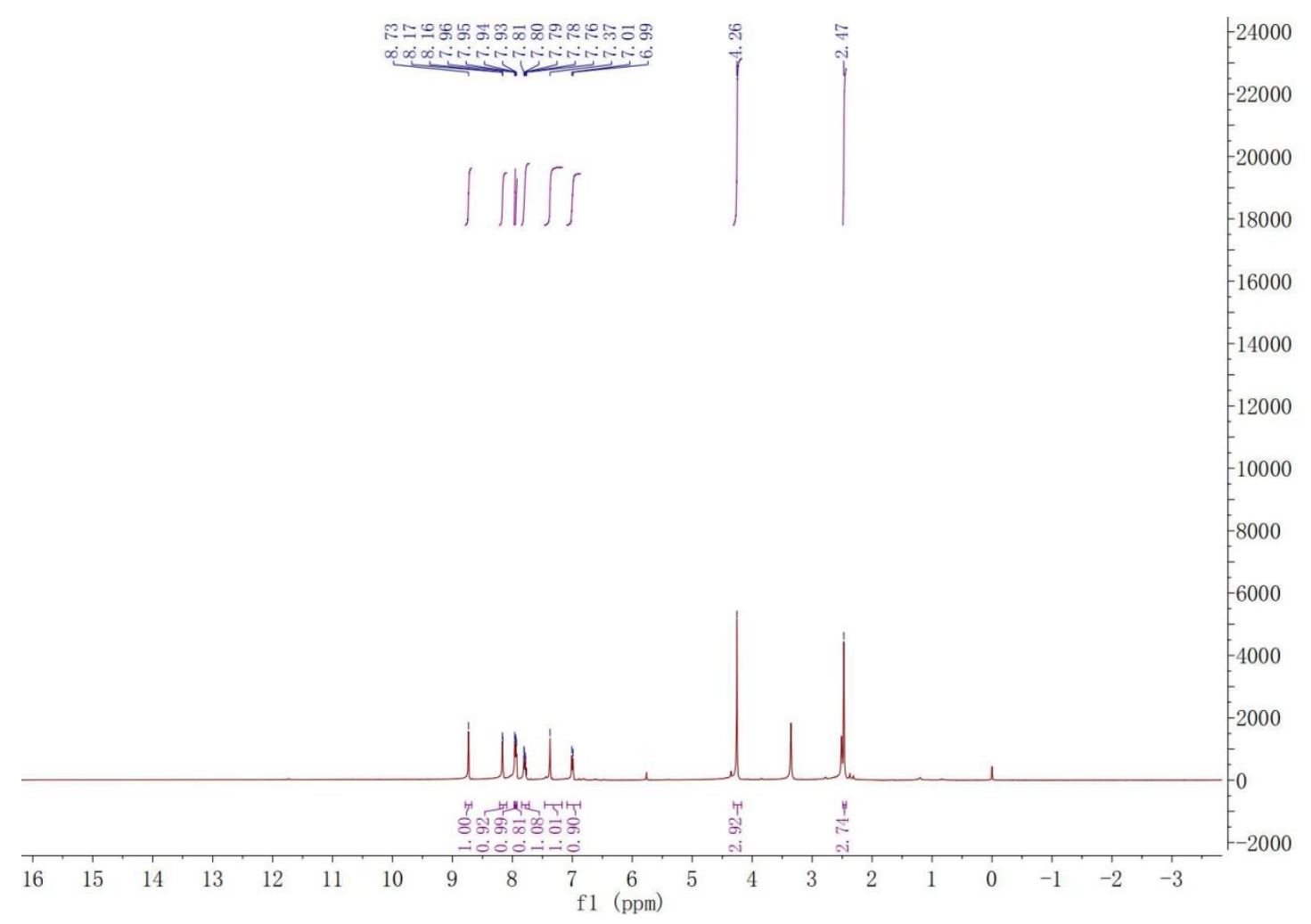

The ${ }^{1} \mathrm{H}$ NMR spectrogram of compound $\mathbf{4 8}$

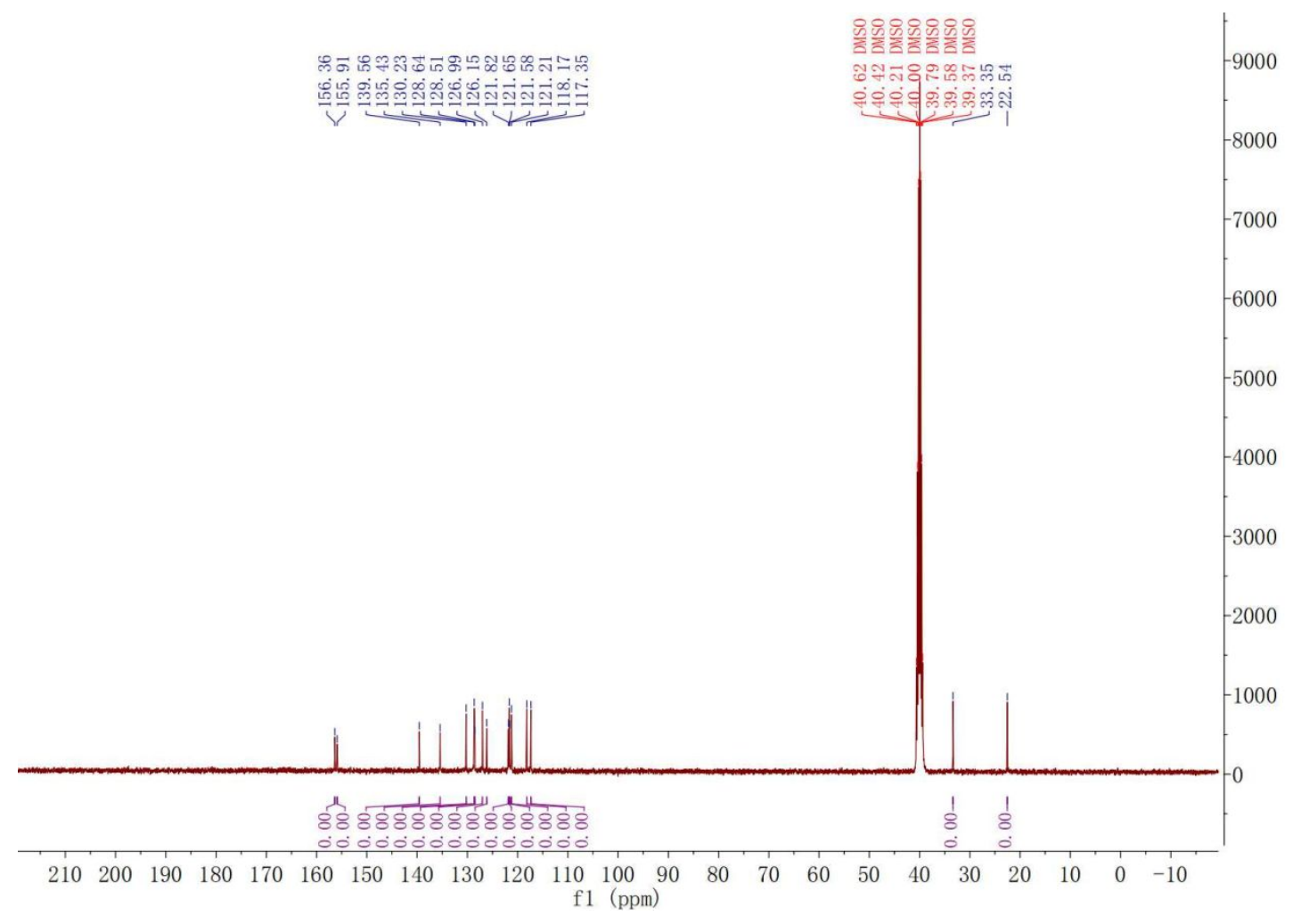

The ${ }^{13} \mathrm{C}$ NMR spectrogram of compound $\mathbf{4 8}$ 


\section{Generic Display Report}

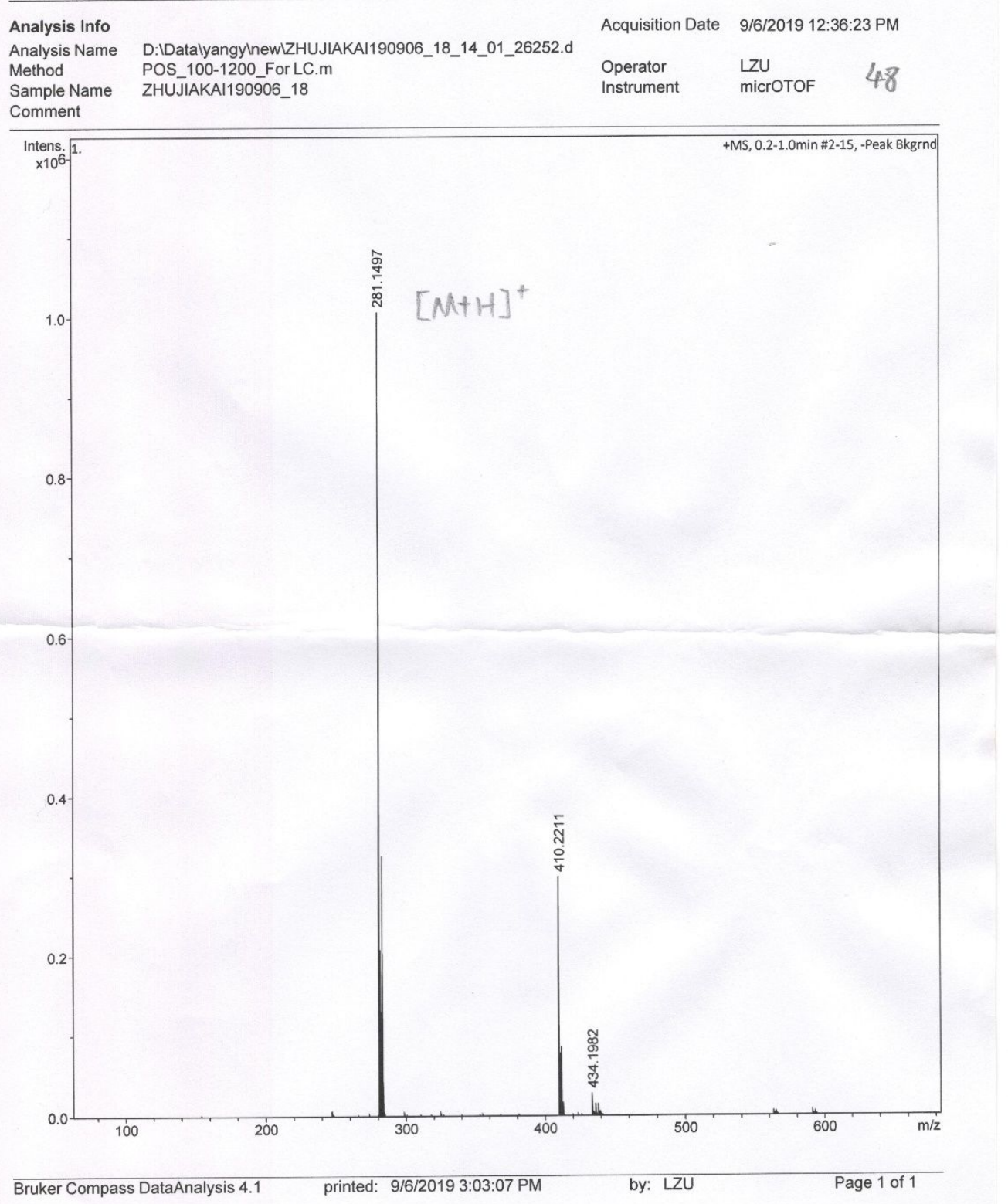

The Mass spectrogram of compound $\mathbf{4 8}$ 